\title{
A Base-Sugar-Phosphate Three Layer ONIOM Model for Cation Binding: Relative Binding Affinities of Alkali Metal Ions for Phosphate Anion in DNA
}

\author{
Neethu Sundaresan ${ }^{a}$ and Cherumuttathu H. Suresh,
}

${ }^{a}$ Chemical Sciences and Technology Division and ${ }^{b}$ Computational Modeling and Simulation Section, Regional Research Laboratory (Council of Scientific and Industrial Research), Trivandrum 695019, India.

\section{Supporting Information}

1. Hydrated Metal ions $\quad$ S2

2. Optimized geometries of tetrahydrated metal ions at B3LYP/6-31G(d) level. $\quad$ S3

3. The P-O bond lengths in the metal-bound DNA fragment systems. S5

4. Optimized geometries at the ONIOM(B3LYP/6-31G(d):PM3:UFF) level. S6

$\begin{array}{ll}\text { 5. Higher coordination geometries } & \text { S47 }\end{array}$ 


\section{Hydrated Metal ions}

The optimized geometries of hydrated $\mathrm{Li}^{+}$in the presence of 4,5 or 6 water molecules suggests that $\mathrm{Li}^{+}$coordinates tetrahedrally to four water molecules, which is the primary solvation shell and the extra water molecules are hydrogen bonded to the water molecules in the primary solvation shell (Figure 1).

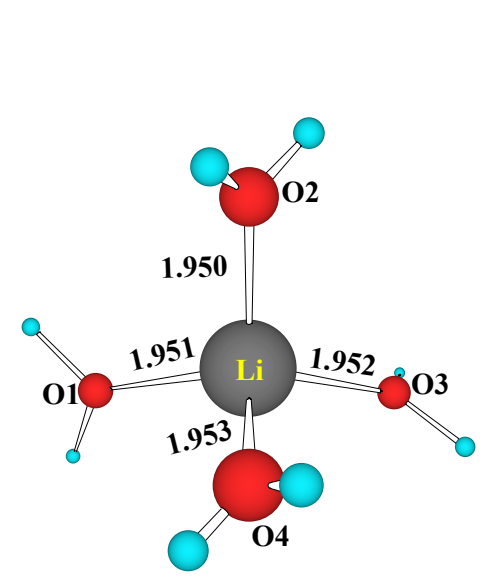

$\mathrm{Li}\left(\mathrm{H}_{2} \mathrm{O}\right)_{4}$

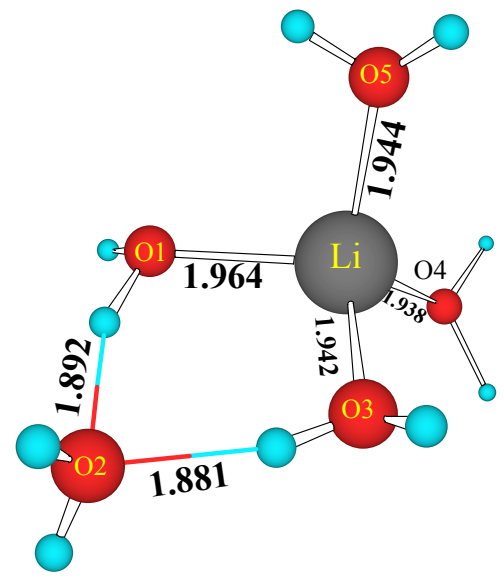

$\mathrm{Li}\left(\mathrm{H}_{2} \mathrm{O}\right)_{5}$

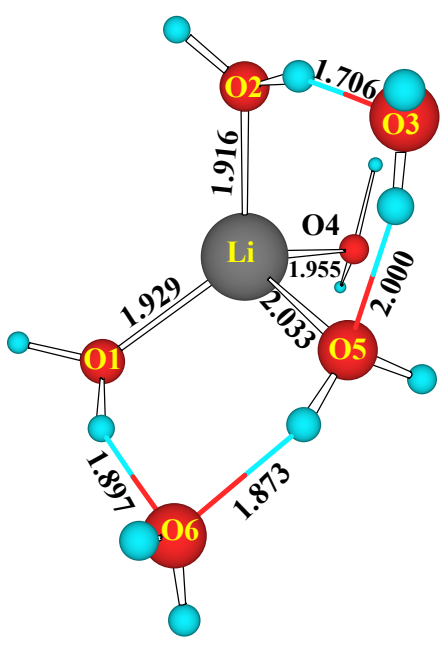

$\mathrm{Li}\left(\mathrm{H}_{2} \mathrm{O}\right)_{6}$

Figure 1. B3LYP optimized geometries of hydrated lithium
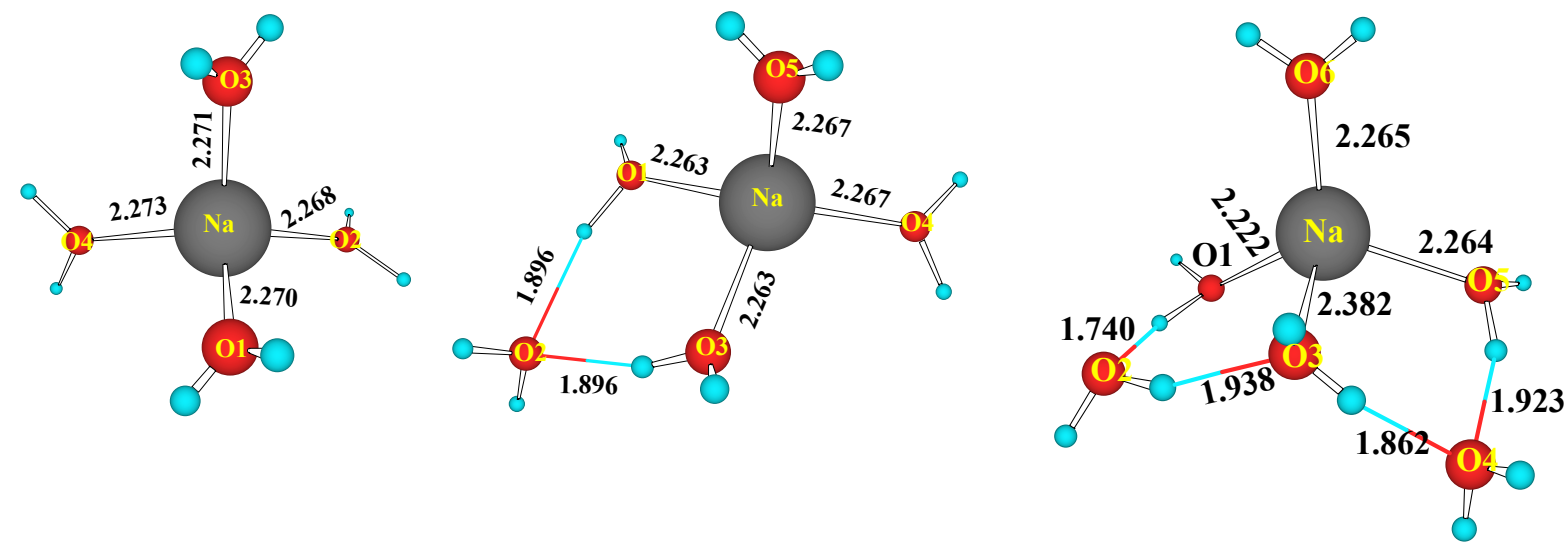

$\mathrm{Na}\left(\mathrm{H}_{2} \mathrm{O}\right)_{4}$

$\mathrm{Na}\left(\mathrm{H}_{2} \mathrm{O}\right)_{5}$

$\mathrm{Na}\left(\mathrm{H}_{2} \mathrm{O}\right)_{6}$

Figure 2. B3LYP optimized geometries of hydrated sodium 
Like $\mathrm{Li}^{+}, \mathrm{Na}^{+}$also affords a tetrahedral geometry with four water molecules (Figure 2), and the additional water molecules are hydrogen bonded to the water molecules in the primary solvation shell. Our results for $\mathrm{Li}^{+}$and $\mathrm{Na}^{+}$are in good agreement with the results obtained by Glendening et al (Glendening, E. D.; Feller, D. J. Phys. Chem. 1995, 99, 3060).
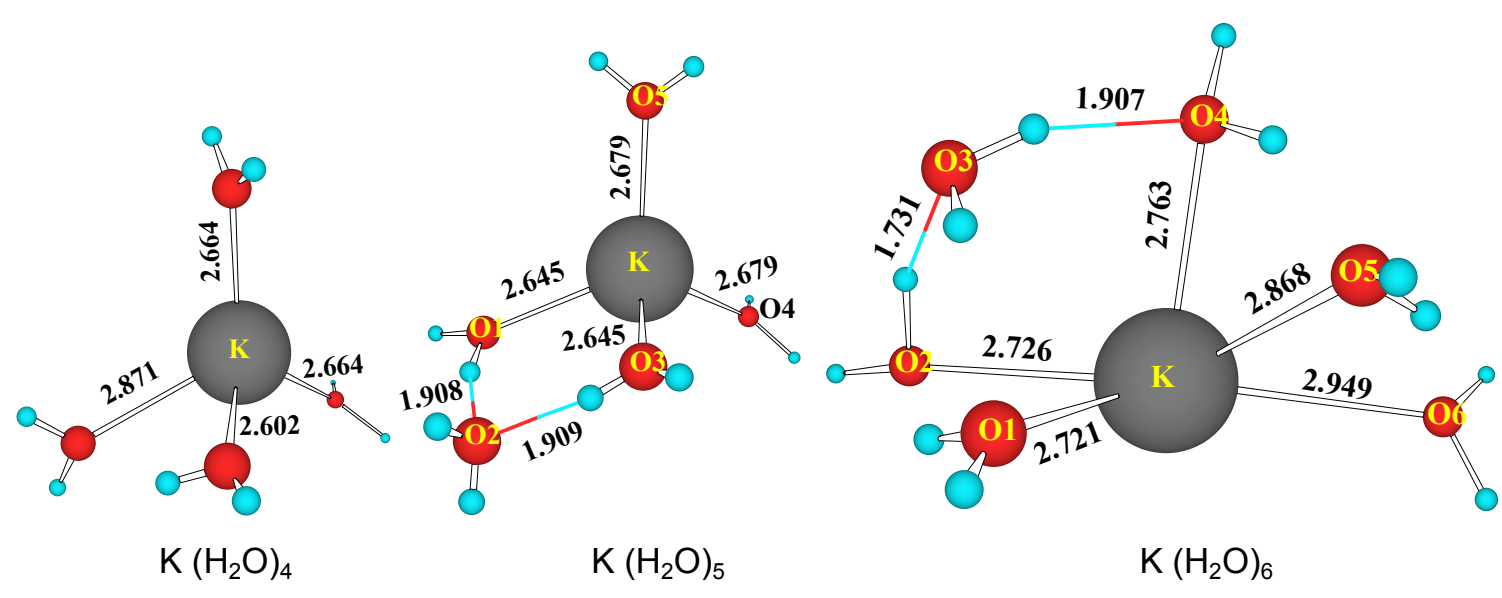

Figure 3. B3LYP optimized geometries of hydrated potassium.

In the case of $\mathrm{K}^{+}$, with 4 and 5 water molecules, the primary solvation shell is found to be tetrahedral, while a sixth water molecule distorts this tetrahedral arrangement in such a way that the primary solvation shell appears as a distorted square pyramid containing five water molecules (Figure 3). In this structure, the sixth one is interacting with two water molecules of the primary solvation shell. We also optimized an octahedral geometry for the hexa hydration, but this was found to be $11.4 \mathrm{kcal} / \mathrm{mol}$ less stable than the square pyramidal structure.

\section{$\underline{\text { 2. Optimized geometries of tetrahydrated metal ions at B3LYP/6-31G(d) level. }}$}

B3LYP/6-31G(d) level optimized geometry of $\operatorname{Li}\left(\mathrm{H}_{2} \mathrm{O}\right)_{4}$. For all the geometries, the atomic number followed by the $\mathrm{X}, \mathrm{Y}$, and $\mathrm{Z}$ coordinates (in $\AA$ ) are presented.

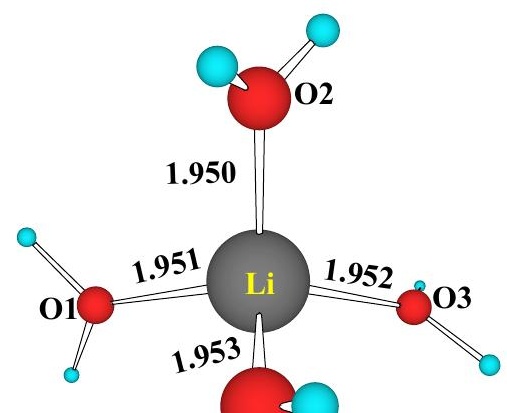




$\begin{array}{rrrr}3 & 0.000447000 & -0.019292000 & -0.007166000 \\ 8 & 1.492239000 & -1.278087000 & -0.006794000 \\ 8 & -0.370932000 & 0.538722000 & 1.824438000 \\ 8 & 0.485611000 & 1.570780000 & -1.028039000 \\ 8 & -1.604268000 & -0.819794000 & -0.780149000 \\ 1 & 2.435373000 & -1.054415000 & -0.027670000 \\ 1 & 1.437408000 & -2.220524000 & -0.228079000 \\ 1 & -1.213706000 & 0.868275000 & 2.172415000 \\ 1 & 0.157843000 & 0.287708000 & 2.597533000 \\ 1 & 0.473936000 & 2.468734000 & -0.662400000 \\ 1 & 0.901866000 & 1.641514000 & -1.900550000 \\ 1 & -2.024454000 & -0.535528000 & -1.606370000 \\ 1 & -2.190812000 & -1.490860000 & -0.399026000\end{array}$

$\underline{\text { B3LYP/6-31G(d) level optimized geometry of } \mathrm{Na}\left(\mathrm{H}_{2}\right.} \underline{\mathrm{O}}_{4}$

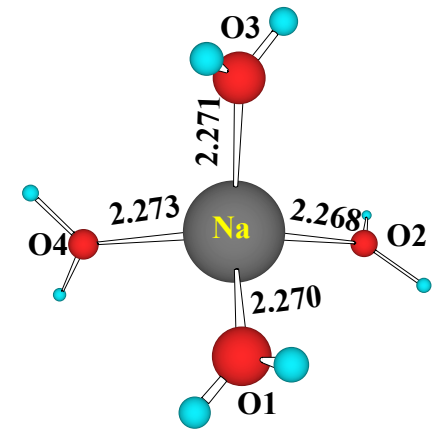

$\begin{array}{lrrr}11 & 0.001092000 & -0.018136000 & -0.012851000 \\ 8 & 1.813914000 & -1.260827000 & -0.571137000 \\ 8 & -0.329731000 & -0.130536000 & 2.231270000 \\ 1 & 2.747434000 & -1.007998000 & -0.504752000 \\ 1 & 1.812967000 & -2.100001000 & -1.056532000 \\ 1 & -1.141914000 & 0.073607000 & 2.719772000 \\ 1 & 0.259389000 & -0.544362000 & 2.880681000 \\ 8 & 0.386380000 & 2.152399000 & -0.564452000 \\ 1 & 0.351743000 & 2.889965000 & 0.063898000 \\ 1 & 0.669933000 & 2.546006000 & -1.403622000\end{array}$




$\begin{array}{llll}8 & -1.869665000 & -0.740015000 & -1.076642000 \\ 1 & -2.357778000 & -0.260272000 & -1.763366000 \\ 1 & -2.360974000 & -1.565628000 & -0.947025000\end{array}$

\section{$\underline{\text { B3LYP/6-31G(d) level optimized geometry of } \mathrm{K}\left(\mathrm{H}_{2}\right.} \underline{\mathrm{O}}_{4}$}

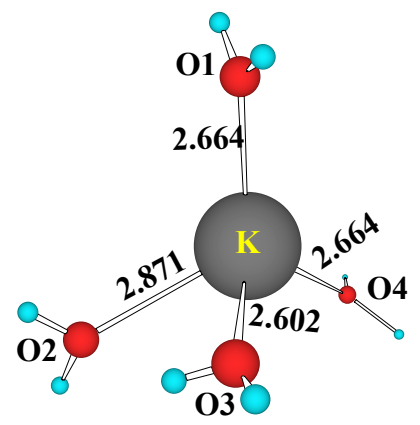

$\begin{array}{lrrr}8 & -2.183779000 & -0.168878000 & -1.278613000 \\ 19 & 0.376937000 & 0.017741000 & 0.006472000 \\ 8 & 1.914087000 & -2.155281000 & -0.098639000 \\ 8 & -1.745710000 & -0.196599000 & 1.495831000 \\ 8 & 1.371718000 & 2.487981000 & -0.057019000 \\ 1 & -2.626291000 & 0.578053000 & -1.713544000 \\ 1 & -2.502333000 & -0.952172000 & -1.755942000 \\ 1 & 2.020738000 & -2.793864000 & 0.623640000 \\ 1 & 2.515353000 & -2.473153000 & -0.789923000 \\ 1 & -2.385122000 & -0.235253000 & 0.761923000 \\ 1 & -2.258506000 & -0.261771000 & 2.314308000 \\ 1 & 1.336600000 & 3.115531000 & 0.681745000 \\ 1 & 1.887234000 & 2.947769000 & -0.737659000\end{array}$

\section{The P-O bond lengths in the metal-bound DNA fragment systems.}

Table 1. Metal-oxygen bond lengths in anion models for hydrated alkali metal ions (Outer sphere). All bond lengths in Angstrom unit. 


\begin{tabular}{|c|c|c|c|c|c|c|c|c|}
\hline \multirow{2}{*}{$\begin{array}{l}\text { Model } \\
\text { system }\end{array}$} & \multicolumn{4}{|c|}{ Outer (Anion) } & \multicolumn{4}{|c|}{ Outer (Dianion) } \\
\hline & 05 & O6 & O7 & 08 & $\mathbf{0 5}$ & O6 & O7 & 08 \\
\hline $\mathrm{Li}^{+}$ & 1.983 & 1.980 & 1.921 & 2.0 & $\begin{array}{l}\text { Not } \\
\text { found }\end{array}$ & $\begin{array}{l}\text { Not } \\
\text { found }\end{array}$ & $\begin{array}{l}\text { Not } \\
\text { found }\end{array}$ & $\begin{array}{l}\text { Not } \\
\text { found }\end{array}$ \\
\hline $\mathrm{Na}^{+}$ & 2.466 & 2.238 & 2.207 & 2.241 & 1.838 & 1.738 & 1.820 & 1.947 \\
\hline $\mathrm{K}^{+}$ & $\begin{array}{l}\text { Not } \\
\text { found }\end{array}$ & $\begin{array}{l}\text { Not } \\
\text { found }\end{array}$ & $\begin{array}{l}\text { Not } \\
\text { found }\end{array}$ & $\begin{array}{l}\text { Not } \\
\text { found }\end{array}$ & 2.715 & 2.762 & 2.806 & 2.722 \\
\hline
\end{tabular}

Table 2. Metal-oxygen bond lengths in anion and dianion models for hydrated alkali metal ions (Inner sphere-monodentate). All bond lengths in Angstrom unit.

\begin{tabular}{lllllllll}
\hline Model & \multicolumn{3}{c}{ Inner mono (Anion) } & \multicolumn{5}{c}{ Inner mono (Dianion) } \\
\cline { 2 - 9 } system & $\mathbf{O 1}$ & $\mathbf{O 5}$ & $\mathbf{O 6}$ & $\mathbf{O} 7$ & O1 & O5 & O6 & O7 \\
\hline $\mathrm{Li}+$ & 2.010 & 1.950 & 1.961 & 1.985 & 1.962 & 1.942 & 1.972 & 1.981 \\
$\mathrm{Na}+$ & 2.335 & 2.305 & 2.296 & 2.272 & 2.303 & 2.251 & 2.296 & 2.313 \\
& & & & & & & & \\
\hline $\mathrm{K}+$ & 2.711 & 2.643 & 2.701 & 2.719 & 2.672 & 2.642 & 2.657 & 2.686 \\
\hline
\end{tabular}

Table 3. Metal-oxygen bond lengths in anion and dianion models for hydrated alkali metal ions (Inner sphere-bidentate). All bond lengths in Angstrom unit.

\begin{tabular}{lllllllll}
\hline Model & \multicolumn{2}{l}{ Inner bi (Anion) } & \multicolumn{5}{l}{ Inner bi (Dianion) } \\
\cline { 2 - 9 } system & $\mathbf{O 1}$ & $\mathbf{O 2}$ & $\mathbf{O 5}$ & $\mathbf{O 6}$ & $\mathbf{O 1}$ & $\mathbf{O 2}$ & $\mathbf{O 5}$ & $\mathbf{O 6}$ \\
\hline $\mathrm{Li}+$ & 2.141 & 2.160 & 1.933 & 1.912 & 2.215 & 2.031 & 1.923 & 1.927 \\
$\mathrm{Na}+$ & 2.439 & 2.364 & 2.256 & 2.238 & 2.439 & 2.364 & 2.256 & 2.238 \\
\hline $\mathrm{K}+$ & 2.770 & 2.728 & 2.616 & 2.649 & Not found & Not found & Not found & Not found \\
\hline
\end{tabular}

Table 4. P-O distances in anion models. All bond lengths in Angstrom unit.

\begin{tabular}{llllll}
\hline Mode & Metal & P-O1 & P-O2 & P-O3 & P-O4 \\
\hline
\end{tabular}




\begin{tabular}{llllll}
\hline Outer & $\mathrm{Li}$ & 1.513 & 1.524 & 1.664 & 1.644 \\
Mono- & $\mathrm{Li}$ & 1.528 & 1.498 & 1.685 & 1.642 \\
$\mathrm{Bi}-$ & $\mathrm{Li}$ & 1.526 & 1.519 & 1.636 & 1.766 \\
Outer & $\mathrm{Na}$ & 1.579 & 1.593 & 1.716 & 1.695 \\
Mono- & $\mathrm{Na}$ & 1.524 & 1.498 & 1.691 & 1.644 \\
$\mathrm{Bi}-$ & $\mathrm{Na}$ & 1.516 & 1.516 & 1.649 & 1.654 \\
Mono & $\mathrm{K}$ & 1.52 & 1.513 & 1.661 & 1.647 \\
Bi- & $\mathrm{K}$ & 1.514 & 1.514 & 1.655 & 1.652 \\
\hline
\end{tabular}

Table 5. P-O distances in dianion models. All bond lengths in Angstrom unit.

\begin{tabular}{llrrrr}
\hline Mode & Metal & P-O1 & P-O2 & P-O3 & P-O4 \\
\hline Mono- & $\mathrm{Li}$ & 1.499 & 1.523 & 1.684 & 1.637 \\
Bi- & $\mathrm{Li}$ & 1.520 & 1.517 & 1.652 & 1.642 \\
Outer & $\mathrm{Na}$ & 1.519 & 1.522 & 1.647 & 1.640 \\
Mono- & $\mathrm{Na}$ & 1.504 & 1.520 & 1.682 & 1.638 \\
Outer & $\mathrm{K}$ & 1.518 & 1.518 & 1.655 & 1.640 \\
Mono- & $\mathrm{K}$ & 1.502 & 1.515 & 1.687 & 1.643 \\
\hline
\end{tabular}

Table 6. Hydrogen bond lengths for GC base pair in anion models for hydrated alkali metal ions.

\begin{tabular}{lcccccccccc}
\hline Model & \multicolumn{3}{c}{ Outer } & \multicolumn{4}{c}{ Inner monodendate } & \multicolumn{3}{c}{ Inner bidendate } \\
\cline { 2 - 9 } system & $\mathrm{N} 4(\mathrm{C})-$ & $\mathrm{N} 3(\mathrm{C})-$ & $\mathrm{O} 2(\mathrm{C})-$ & $\mathrm{N} 4(\mathrm{C})-$ & $\mathrm{N} 3(\mathrm{C})-$ & $\mathrm{O} 2(\mathrm{C})-$ & $\mathrm{N} 4(\mathrm{C})-$ & $\mathrm{N} 3(\mathrm{C})-$ & $\mathrm{O} 2(\mathrm{C})-$ \\
& $\mathrm{O} 6(\mathrm{G})$ & $\mathrm{N} 1(\mathrm{G})$ & $\mathrm{N} 2(\mathrm{G})$ & $\mathrm{O} 6(\mathrm{G})$ & $\mathrm{N} 1(\mathrm{G})$ & $\mathrm{N} 2(\mathrm{G})$ & $\mathrm{O} 6(\mathrm{G})$ & $\mathrm{N} 1(\mathrm{G})$ & $\mathrm{N} 2(\mathrm{G})$ \\
\hline $\mathrm{Li}^{+}$ & 1.797 & 1.787 & 1.832 & 1.796 & 1.787 & 1.831 & 1.798 & 1.778 & 1.831 \\
$\mathrm{Na}^{+}$ & 1.809 & 1.779 & 1.820 & 1.809 & 1.777 & 1.819 & 1.808 & 1.779 & 1.822 \\
$\mathrm{~K}^{+}$ & $\begin{array}{l}\text { Not } \\
\text { found }\end{array}$ & $\begin{array}{l}\text { Not } \\
\text { found }\end{array}$ & $\begin{array}{l}\text { Not } \\
\text { found }\end{array}$ & 1.807 & 1.781 & 1.822 & 1.808 & 1.778 & 1.823 \\
\hline
\end{tabular}


Table 6. GC base pair hydrogen bond lengths in dianion models for hydrated alkali metal ions.

\begin{tabular}{llllllllll}
\hline \multirow{2}{*}{$\begin{array}{l}\text { Model } \\
\text { system }\end{array}$} & Outer & & \multicolumn{4}{c}{ Inner monodendate } & \multicolumn{3}{l}{ Inner bidendate } \\
\cline { 2 - 10 } & N4(C)- & N3(C)- & O2(C)- & N4(C)- & N3(C)- & O2(C)- & N4(C)- & N3(C)- & O2(C)- \\
& O6(G) & N1(G) & N2(G) & O6(G) & N1(G) & N2(G) & O6(G) & N1(G) & N2(G) \\
\hline $\mathrm{Li}+$ & $\begin{array}{l}\text { Not } \\
\text { found }\end{array}$ & $\begin{array}{l}\text { Not } \\
\text { found }\end{array}$ & $\begin{array}{l}\text { Not } \\
\text { found }\end{array}$ & 1.784 & 1.801 & 1.852 & 1.785 & 1.801 & 1.854 \\
$\mathrm{Na}^{+}$ & 1.797 & 1.794 & 1.840 & 1.796 & 1.796 & 1.841 & 1.798 & 1.792 & 1.842 \\
$\mathrm{~K}+$ & 1.796 & 1.795 & 1.840 & 1.798 & 1.793 & 1.842 & $\begin{array}{l}\text { Not } \\
\text { found }\end{array}$ & $\begin{array}{l}\text { Not } \\
\text { found }\end{array}$ & $\begin{array}{l}\text { Not } \\
\text { found }\end{array}$ \\
\hline
\end{tabular}

\section{Optimized geometries at the ONIOM(B3LYP/6-31G(d):PM3:UFF) level.}

See Scheme 1 and 2 in the article for the details regarding the selection of high, medium and low level of theory.

Bare anion

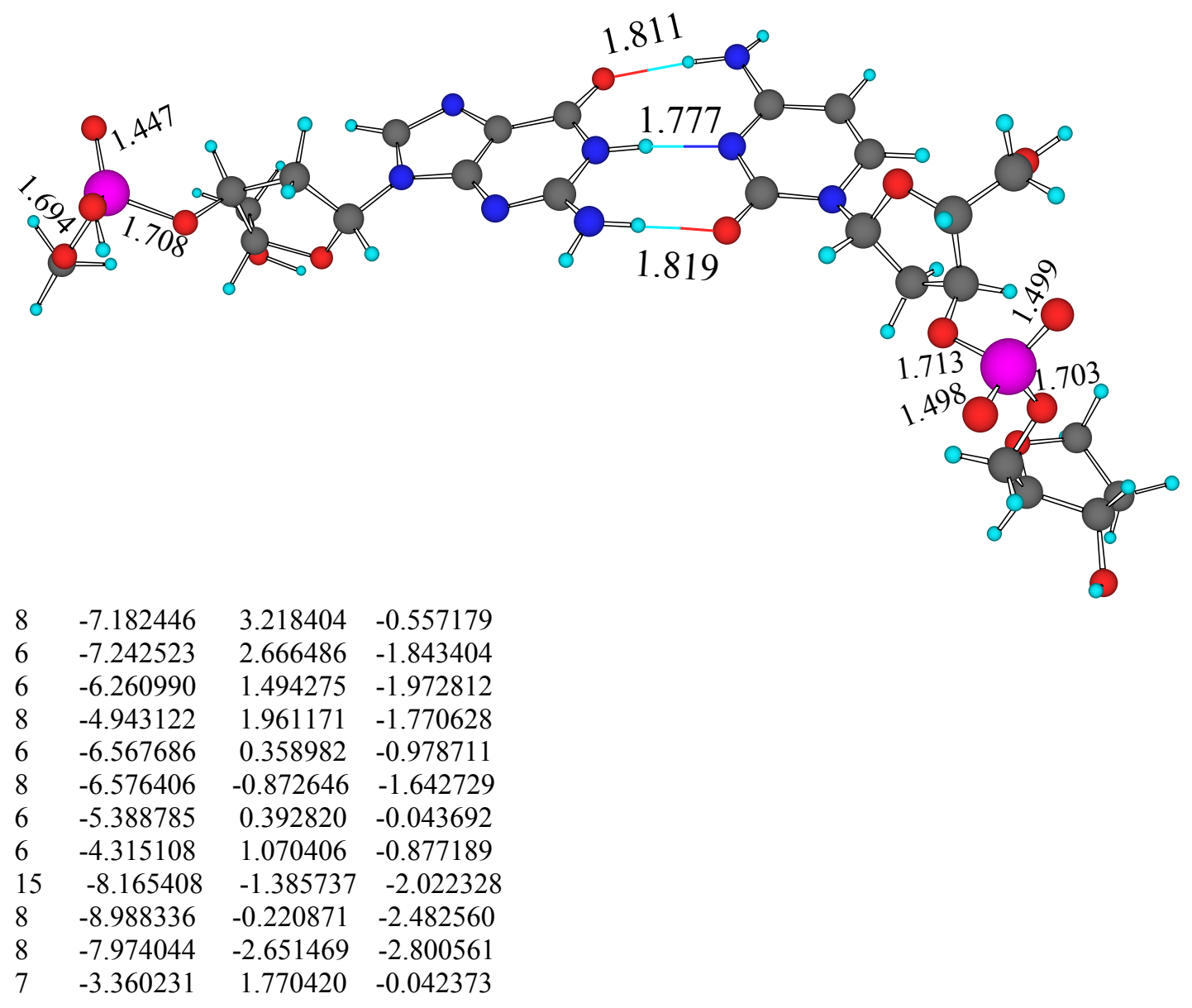




\begin{tabular}{lccc}
6 & -1.944804 & 1.541962 & -0.267972 \\
8 & -1.518835 & 0.511665 & -0.780018 \\
7 & -1.012821 & 2.500471 & 0.195771 \\
6 & -1.453720 & 3.626100 & 0.793797 \\
7 & -0.489635 & 4.559228 & 1.125662 \\
6 & -2.859914 & 3.885478 & 0.985193 \\
6 & -3.771029 & 2.986883 & 0.521451 \\
8 & -8.726947 & -1.719502 & -0.449222 \\
6 & -8.189605 & -2.914701 & 0.028144 \\
6 & -8.447179 & -3.031273 & 1.534030 \\
8 & -7.885357 & -1.921346 & 2.202694 \\
6 & -9.937738 & -3.119947 & 1.869191 \\
8 & -10.349173 & -4.448999 & 2.051939 \\
6 & -10.027112 & -2.356385 & 3.155369 \\
6 & -8.878380 & -1.377200 & 3.041312 \\
1 & -7.835193 & 3.965692 & -0.547381 \\
1 & -6.978951 & 3.443471 & -2.595523 \\
1 & -8.276370 & 2.314645 & -2.054224 \\
1 & -6.335760 & 1.112316 & -3.015679 \\
1 & -7.492990 & 0.542169 & -0.380939 \\
1 & -5.073332 & -0.623450 & 0.281886 \\
1 & -5.644513 & 0.991614 & 0.857747 \\
1 & -3.815205 & 0.282031 & -1.485048 \\
1 & -0.771075 & 5.232576 & 1.796517 \\
1 & 0.475116 & 4.260440 & 1.181869 \\
1 & -3.186555 & 4.807441 & 1.477628 \\
1 & -4.844282 & 3.193171 & 0.634517 \\
1 & -8.659759 & -3.779723 & -0.490337 \\
1 & -7.086488 & -2.955925 & -0.125127 \\
1 & -7.932553 & -3.946203 & 1.905256 \\
1 & -10.571331 & -2.614152 & 1.102504 \\
1 & -10.419013 & -4.846657 & 1.145235 \\
1 & -9.858813 & -3.029337 & 4.026169 \\
1 & -11.003965 & -1.836229 & 3.262477 \\
1 & -9.237036 & -0.422234 & 2.598680 \\
1 & -8.448573 & -1.164294 & 4.043179 \\
7 & 5.590516 & 0.554290 & 0.562534 \\
6 & 6.086025 & 1.622073 & 1.365197 \\
7 & 5.106728 & 2.499280 & 1.599473 \\
6 & 3.946710 & 2.042752 & 0.954052 \\
6 & 2.614680 & 2.592949 & 0.883756 \\
8 & 2.151812 & 3.610971 & 1.396666 \\
7 & 1.703971 & 1.814642 & 0.089343 \\
6 & 2.096759 & 0.611865 & -0.534822 \\
7 & 1.190672 & -0.018638 & -1.390679 \\
7 & 3.343436 & 0.081246 & -0.444643 \\
6 & 4.237894 & 0.830907 & 0.304469 \\
6 & 12.080355 & -2.560943 & 0.704260 \\
6 & 8.879935 & -0.327481 & 2.287236 \\
6 & 8.354754 & -1.203915 & 1.128206 \\
8 & 6.929695 & -1.274071 & 1.167652 \\
6 & 8.787559 & -0.632605 & -0.255593 \\
8 & 9.442587 & -1.620117 & -1.003142 \\
6 & 7.463362 & -0.231793 & -0.939157 \\
6 & 6.349057 & -0.610373 & 0.053263 \\
15 & 11.072042 & -1.463902 & -1.490538 \\
& & & \\
\hline
\end{tabular}




$\begin{array}{cccc}8 & 10.979292 & -2.152688 & -2.993869 \\ 8 & 11.675075 & -0.153182 & -1.378728 \\ 8 & 11.775368 & -2.747763 & -0.638800 \\ 8 & 8.695475 & -0.918141 & 3.541976 \\ 1 & 7.11272 & 1.686135 & 1.751673 \\ 1 & 0.711238 & 2.095119 & 0.050952 \\ 1 & 1.412770 & -0.959628 & -1.619028 \\ 1 & 0.209476 & 0.146151 & -1.212374 \\ 1 & 12.427189 & -3.535180 & 1.061968 \\ 1 & 11.196330 & -2.254150 & 1.280909 \\ 1 & 12.878333 & -1.819588 & 0.831508 \\ 1 & 9.977105 & -0.217384 & 2.221292 \\ 1 & 8.427236 & 0.689466 & 2.259632 \\ 1 & 8.668319 & -2.263862 & 1.275324 \\ 1 & 9.459433 & 0.250925 & -0.134247 \\ 1 & 7.314720 & -0.763284 & -1.898951 \\ 1 & 7.457661 & 0.845132 & -1.193241 \\ 1 & 5.618509 & -1.340859 & -0.368960 \\ 1 & 10.545581 & -2.990313 & -3.047793 \\ 1 & 7.761277 & -1.033280 & 3.667909\end{array}$

\section{Bare dianion}

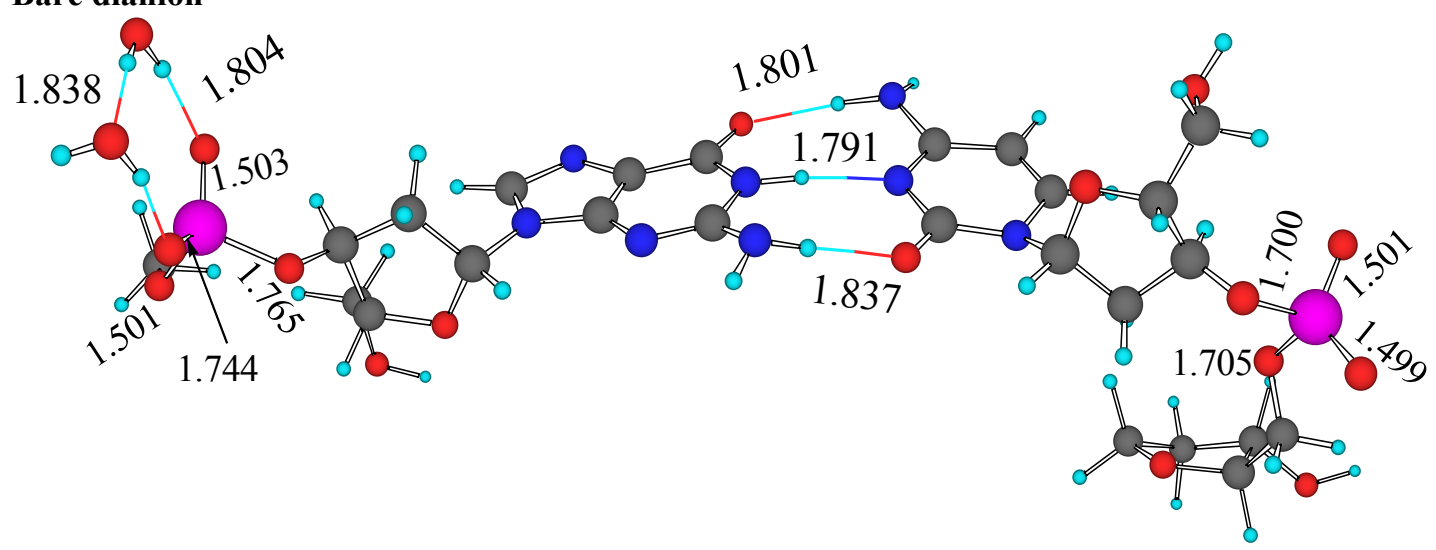
$\begin{array}{llll}\text { O } & 8.879883 & 3.451660 & 1.325836\end{array}$
$\begin{array}{llll}\text { C } & 9.006454 & 2.807449 & 2.564377\end{array}$
$\begin{array}{llll}\text { C } & 8.216171 & 1.492432 & 2.569565\end{array}$
$\begin{array}{llll}\mathrm{O} & 6.876556 & 1.731216 & 2.208847\end{array}$
C $\quad 8.786102 \quad 0.470505 \quad 1.632172$
$\begin{array}{llll}\text { O } & 9.778336 & -0.250824 & 2.171021\end{array}$
$\begin{array}{llll}\text { C } & 7.603134 & -0.342758 & 1.251190\end{array}$
$\begin{array}{llll}\text { C } & 6.407227 & 0.550446 & 1.595978\end{array}$
$\begin{array}{llll}\mathrm{P} & 11.097656 & -0.580688 & 1.151382\end{array}$
$\begin{array}{lllll}\text { O } & 11.617065 & 0.699158 & 0.564926\end{array}$
$\begin{array}{llll}\text { O } & 11.947037 & -1.556625 & 1.908417\end{array}$
$\begin{array}{llll}\mathrm{N} & 5.616165 & 0.878372 & 0.425858\end{array}$
$\begin{array}{llll}\text { C } & 4.184784 & 1.057114 & 0.626968\end{array}$
$\begin{array}{llll}\mathrm{O} & 3.574631 & 0.472321 & 1.518082\end{array}$
$\begin{array}{llll}\mathrm{N} & 3.448990 & 1.853210 & -0.279953\end{array}$ 


\begin{tabular}{|c|c|c|c|}
\hline $\mathrm{C}$ & 4.092758 & 2.498810 & -1.273422 \\
\hline $\mathrm{N}$ & 3.323639 & 3.334747 & -2.058640 \\
\hline $\mathrm{C}$ & 5.523575 & 2.402374 & -1.439041 \\
\hline $\mathrm{C}$ & 6.248978 & 1.656662 & -0.564346 \\
\hline $\mathrm{O}$ & 10.246689 & -1.348450 & -0.110809 \\
\hline $\mathrm{C}$ & 10.481949 & -2.654526 & -0.241516 \\
\hline C & 9.676712 & -3.333893 & -1.314468 \\
\hline $\mathrm{O}$ & 8.299210 & -3.178482 & -1.047333 \\
\hline $\mathrm{C}$ & 9.978775 & -2.778152 & -2.706558 \\
\hline $\mathrm{O}$ & 10.947449 & -3.545334 & -3.371475 \\
\hline $\mathrm{C}$ & 8.651260 & -2.890488 & -3.392197 \\
\hline $\mathrm{C}$ & 7.670288 & -2.776337 & -2.243622 \\
\hline $\mathrm{H}$ & 9.421951 & 4.280289 & 1.390167 \\
\hline $\mathrm{H}$ & 8.609894 & 3.468400 & 3.367203 \\
\hline $\mathrm{H}$ & 10.079300 & 2.599350 & 2.777924 \\
\hline $\mathrm{H}$ & 8.230545 & 1.087097 & 3.608078 \\
\hline $\mathrm{H}$ & 9.080231 & 0.985489 & 0.720505 \\
\hline $\mathrm{H}$ & 7.564835 & -1.278275 & 1.854324 \\
\hline $\mathrm{H}$ & 7.628906 & -0.632370 & 0.177582 \\
\hline $\mathrm{H}$ & 5.792175 & -0.001495 & 2.341782 \\
\hline $\mathrm{H}$ & 3.727356 & 3.579071 & -2.930313 \\
\hline $\mathrm{H}$ & 2.317886 & 3.215652 & -2.056686 \\
\hline $\mathrm{H}$ & 6.020096 & 2.946198 & -2.249893 \\
\hline $\mathrm{H}$ & 7.342270 & 1.612137 & -0.677994 \\
\hline $\mathrm{H}$ & 11.535202 & -2.862534 & -0.402176 \\
\hline $\mathrm{H}$ & 10.332947 & -3.190613 & 0.690796 \\
\hline $\mathrm{H}$ & 9.905930 & -4.424088 & -1.295700 \\
\hline $\mathrm{H}$ & 10.279526 & -1.703751 & -2.674332 \\
\hline $\mathrm{H}$ & 11.821884 & -3.297043 & -2.973022 \\
\hline $\mathrm{H}$ & 8.542068 & -3.885574 & -3.879500 \\
\hline $\mathrm{H}$ & 8.503128 & -2.084091 & -4.143295 \\
\hline $\mathrm{H}$ & 7.336334 & -1.721329 & -2.136124 \\
\hline $\mathrm{H}$ & 6.778473 & -3.411819 & -2.430023 \\
\hline $\mathrm{N}$ & -3.404251 & 1.088623 & -0.377136 \\
\hline C & -3.671936 & 1.713776 & -1.622971 \\
\hline $\mathrm{N}$ & -2.543137 & 2.228180 & -2.126572 \\
\hline $\mathrm{C}$ & -1.504333 & 1.951324 & -1.225342 \\
\hline C & -0.097107 & 2.259613 & -1.274109 \\
\hline $\mathrm{O}$ & 0.557938 & 2.843185 & -2.140350 \\
\hline $\mathrm{N}$ & 0.639664 & 1.806076 & -0.129547 \\
\hline C & 0.020157 & 1.091705 & 0.921219 \\
\hline $\mathrm{N}$ & 0.795898 & 0.784241 & 2.055283 \\
\hline $\mathrm{N}$ & -1.292404 & 0.782608 & 0.955475 \\
\hline C & -2.032089 & 1.235794 & -0.134186 \\
\hline $\mathrm{C}$ & -6.498672 & -0.292374 & -2.069962 \\
\hline C & -6.294586 & -0.515640 & -0.558014 \\
\hline $\mathrm{O}$ & -4.904495 & -0.703140 & -0.272125 \\
\hline C & -6.823593 & 0.705490 & 0.266357 \\
\hline C & -5.549759 & 1.264954 & 0.954605 \\
\hline $\mathrm{C}$ & -4.388260 & 0.377090 & 0.485382 \\
\hline $\mathrm{O}$ & -6.153229 & -1.398697 & -2.858368 \\
\hline $\mathrm{C}$ & -10.155151 & 0.337250 & -1.266449 \\
\hline $\mathrm{O}$ & -9.322021 & 2.415466 & 0.735367 \\
\hline$P$ & -9.382217 & 1.002121 & 1.241898 \\
\hline $\mathrm{O}$ & -7.762589 & 0.301544 & 1.194550 \\
\hline $\mathrm{O}$ & -9.960495 & 0.634567 & \\
\hline
\end{tabular}




$\begin{array}{cccc}\mathrm{O} & -10.146744 & -0.028671 & 0.060776 \\ \mathrm{H} & -4.662277 & 1.738083 & -2.100769 \\ \mathrm{H} & 1.665024 & 1.898712 & -0.125488 \\ \mathrm{H} & 0.400589 & 0.081802 & 2.637451 \\ \mathrm{H} & 1.784088 & 0.655060 & 1.885025 \\ \mathrm{H} & -7.576935 & -0.142365 & -2.288605 \\ \mathrm{H} & -5.946442 & 0.609451 & -2.420883 \\ \mathrm{H} & -6.753952 & -1.478882 & -0.240128 \\ \mathrm{H} & -7.261856 & 1.474289 & -0.416550 \\ \mathrm{H} & -5.637070 & 1.224686 & 2.057312 \\ \mathrm{H} & -5.400100 & 2.332153 & 0.707031 \\ \mathrm{H} & -3.826447 & -0.095764 & 1.324234 \\ \mathrm{H} & -5.261734 & -1.636093 & -2.634140 \\ \mathrm{H} & -10.832443 & -0.356369 & -1.779114 \\ \mathrm{H} & -9.153427 & 0.241959 & -1.724091 \\ \mathrm{H} & -10.514587 & 1.363007 & -1.421631 \\ \mathrm{O} & -10.552048 & 4.424088 & 2.162109 \\ \mathrm{H} & -10.725143 & 3.927780 & 2.967911 \\ \mathrm{H} & -10.085587 & 3.827789 & 1.557083 \\ \mathrm{O} & -11.043503 & 2.551300 & 4.143295 \\ \mathrm{H} & -11.947037 & 2.281616 & 4.018570 \\ \mathrm{H} & -10.486984 & 1.896133 & 3.684219\end{array}$

\section{$\left.\underline{\mathrm{Li}\left(\mathrm{H}_{2}\right.} \underline{\mathrm{O}}\right)_{4}-$ Outer sphere- Anion Model}

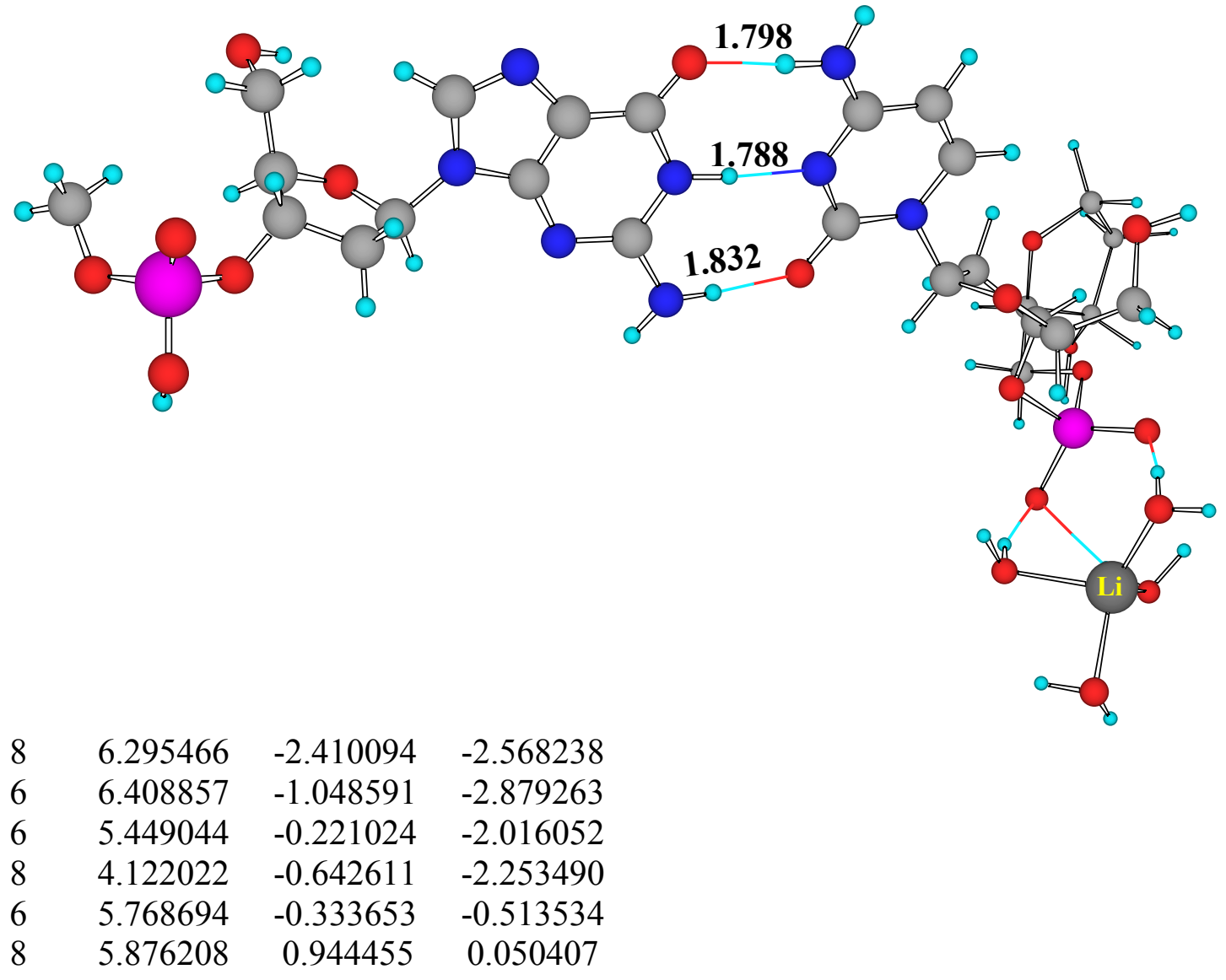




$\begin{array}{lccc}6 & 4.548715 & -1.014005 & 0.050406 \\ 6 & 3.487297 & -0.736277 & -0.999445 \\ 15 & 7.45066 & 1.4083590 & 0.324767 \\ 8 & 8.315908 & 1.183626 & -0.895625 \\ 8 & 7.312135 & 2.824555 & 0.869880 \\ 7 & 2.470325 & -1.768721 & -1.010356 \\ 6 & 1.075659 & -1.348507 & -1.019694 \\ 8 & 0.722840 & -0.279466 & -0.531083 \\ 7 & 0.095206 & -2.229448 & -1.521218 \\ 6 & 0.465488 & -3.426836 & -2.028286 \\ 7 & -0.538558 & -4.188973 & -2.567964 \\ 6 & 1.853461 & -3.836531 & -2.0791260 \\ 6 & 2.812481 & -2.988916 & -1.6285980 \\ 8 & 8.000247 & 0.359997 & 1.4650250 \\ 6 & 7.437619 & 0.608705 & 2.7186960 \\ 6 & 7.764587 & -0.545433 & 3.671973 \\ 8 & 7.291026 & -1.761517 & 3.132714 \\ 6 & 9.263547 & -0.669521 & 3.951044 \\ 8 & 9.623967 & -0.021998 & 5.142696 \\ 6 & 9.446800 & -2.151200 & 4.077333 \\ 6 & 8.340729 & -2.699552 & 3.200670 \\ 1 & 6.939499 & -2.881519 & -3.1578030 \\ 1 & 6.159309 & -0.888984 & -3.9520940 \\ 1 & 7.454137 & -0.707545 & -2.7090710 \\ 1 & 5.535688 & 0.839645 & -2.3415780 \\ 1 & 6.654802 & -0.983598 & -0.3106890 \\ 1 & 4.255712 & -0.608819 & 1.044419 \\ 1 & 4.743052 & -2.103953 & 0.153774 \\ 1 & 3.047211 & 0.261338 & -0.7705470 \\ 1 & -0.323236 & -5.13919 & -2.743809 \\ 1 & -1.504926 & -3.96612 & -2.366256 \\ 1 & 2.120237 & -4.813642 & -2.4969620 \\ 1 & 3.870304 & -3.278902 & -1.6914350 \\ 1 & 7.842657 & 1.555026 & 3.1421260 \\ 1 & 6.327253 & 0.686688 & 2.6564850 \\ 1 & 7.226750 & -0.367457 & 4.630643 \\ 1 & 9.881066 & -0.298542 & 3.098831 \\ 1 & 9.634391 & 0.949593 & 4.9404370 \\ 1 & 9.287401 & -2.480121 & 5.129004 \\ 1 & 10.45081 & -2.476659 & 3.727663 \\ 1 & 8.726566 & -2.879923 & 2.173549 \\ 1 & 7.960372 & -3.659429 & 3.610165 \\ 8 & 5.997243 & 4.239811 & -0.9629410 \\ 3 & 7.675241 & 4.117884 & -2.0434800 \\ 8 & 9.156706 & 4.036010 & -0.7274240 \\ 8 & 7.542938 & 6.046044 & -2.4730460\end{array}$




$\begin{array}{lllc}8 & 7.707679 & 2.516162 & -3.1028990 \\ 1 & 5.263963 & 3.690399 & -1.2814500 \\ 1 & 6.322082 & 3.767002 & -0.1427710 \\ 1 & 9.568763 & 3.179351 & -0.9421360 \\ 1 & 8.612205 & 3.776483 & 0.0601530 \\ 1 & 6.769852 & 6.114288 & -1.8827790 \\ 1 & 8.272735 & 6.458366 & -1.9823060 \\ 1 & 8.352250 & 2.349116 & -3.8055240 \\ 1 & 7.927324 & 1.877709 & -2.3603700 \\ 7 & -6.492374 & -0.80940 & -0.058528 \\ 6 & -7.067671 & -2.05741 & -0.430306 \\ 7 & -6.124406 & -2.852460 & -0.943100 \\ 6 & -4.908747 & -2.151590 & -0.929758 \\ 6 & -3.584772 & -2.533610 & -1.354922 \\ 8 & -3.179943 & -3.594130 & -1.830786 \\ 7 & -2.599145 & -1.501490 & -1.186388 \\ 6 & -2.919388 & -0.245880 & -0.625830 \\ 7 & -1.926213 & 0.74527 & -0.6078490 \\ 7 & -4.156889 & 0.10183 & -0.2046320 \\ 6 & -5.127844 & -0.877280 & -0.378612 \\ 6 & -12.97594 & 1.39425 & 2.2050360 \\ 6 & -9.882882 & -1.401230 & 1.591816 \\ 6 & -9.259846 & 0.01256 & 1.5894350 \\ 8 & -7.839394 & -0.073120 & 1.713348 \\ 6 & -9.599888 & 0.78014 & 0.2762990 \\ 8 & -10.18491 & 2.01581 & 0.5769370 \\ 6 & -8.234995 & 0.98460 & -0.4158560 \\ 6 & -7.191328 & 0.35955 & 0.5265120 \\ 15 & -11.770240 & 2.418700 & 0.074740 \\ 8 & -11.55091 & 4.02366 & -0.2669880 \\ 8 & -12.36496 & 1.57811 & -0.9410160 \\ 8 & -12.54244 & 2.55482 & 1.5743860 \\ 8 & -9.782016 & -2.038430 & 2.832776 \\ 1 & -8.123151 & -2.324470 & -0.285127 \\ 1 & -1.612596 & -1.719270 & -1.384305 \\ 1 & -2.114887 & 1.50689 & 0.0022220 \\ 1 & -0.972757 & 0.41440 & -0.5645990 \\ 1 & -13.36842 & 1.71952 & 3.1732610 \\ 1 & -12.14756 & 0.68975 & 2.3647130 \\ 1 & -13.77239 & 0.90340 & 1.6330680 \\ 1 & -10.97401 & -1.341830 & 1.429348 \\ 1 & -9.446921 & -2.034100 & 0.785871 \\ 1 & -9.564997 & 0.56909 & 2.5062170 \\ 1 & -10.28992 & 0.18531 & -0.3695580 \\ 1 & -8.008982 & 2.05648 & -0.5763920 \\ & -8.227589 & 0.52645 & -1.4230370\end{array}$


$\begin{array}{lllr}1 & -6.418612 & 1.08871 & 0.8675850 \\ 1 & -11.18125 & 4.55563 & 0.4207200 \\ 1 & -8.858340 & -2.132660 & 3.031673\end{array}$

$\mathrm{Na}\left(\mathrm{H}_{2} \mathrm{O}\right)_{4}$-Outer sphere- Anion Model

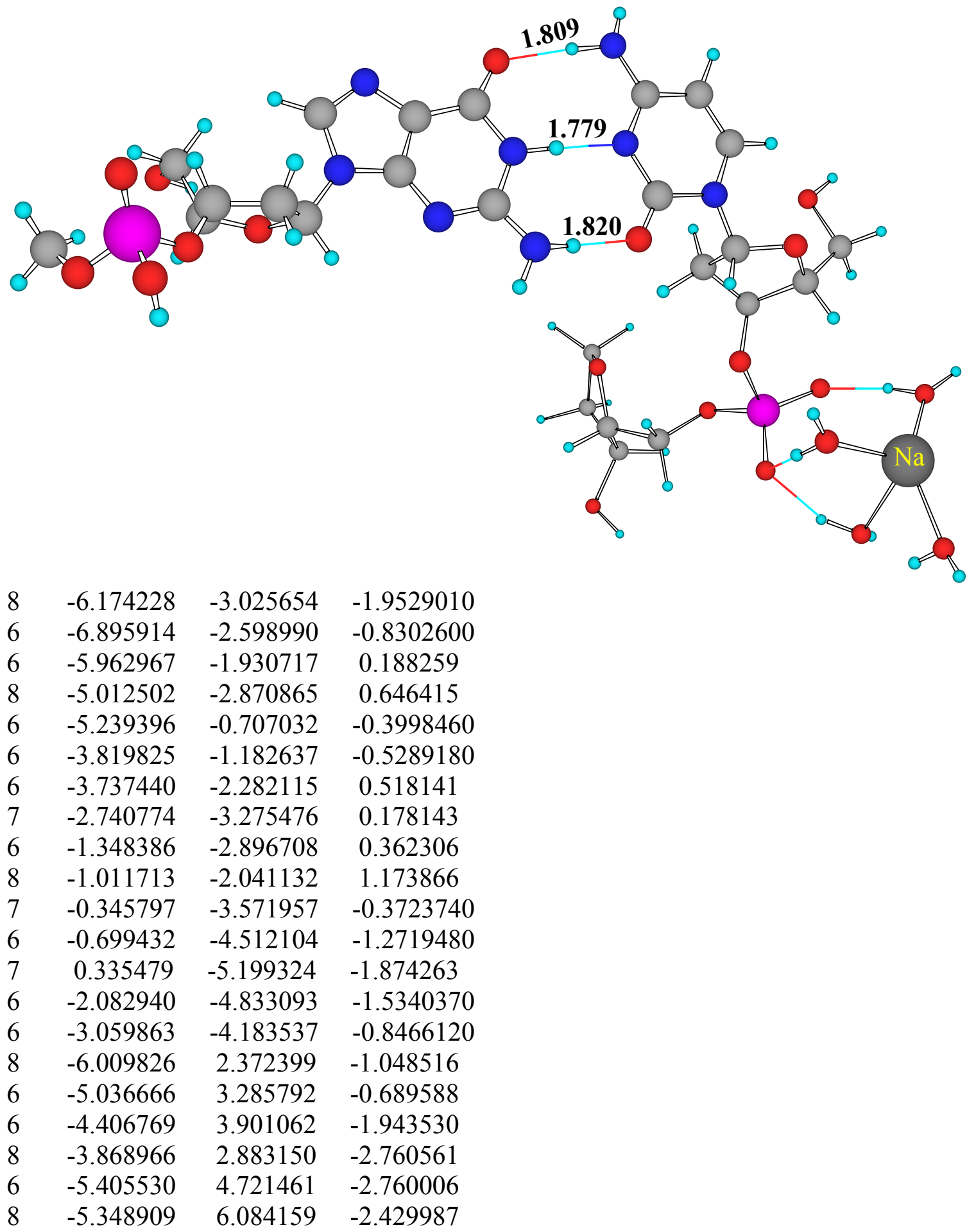




$\begin{array}{llcc}6 & -4.933590 & 4.496294 & -4.164049 \\ 6 & -4.286100 & 3.128951 & -4.084687 \\ 15 & -6.57982 & 1.4407070 & 0.247284 \\ 8 & -7.876215 & 0.663705 & -0.208075 \\ 8 & -6.637695 & 2.292902 & 1.591849 \\ 8 & -5.282847 & 0.345782 & 0.498187 \\ 1 & -6.834849 & -3.449189 & -2.5601750 \\ 1 & -7.390846 & -3.472260 & -0.349395 \\ 1 & -7.684882 & -1.879181 & -1.141835 \\ 1 & -6.587128 & -1.621244 & 1.056307 \\ 1 & -5.617689 & -0.410584 & -1.407414 \\ 1 & -3.079509 & -0.374373 & -0.337709 \\ 1 & -3.656234 & -1.593344 & -1.550154 \\ 1 & -3.488263 & -1.825105 & 1.503475 \\ 1 & 0.096866 & -5.671395 & -2.712539 \\ 1 & 1.265483 & -4.802073 & -1.842074 \\ 1 & -2.337039 & -5.584668 & -2.289083 \\ 1 & -4.112647 & -4.419297 & -1.053807 \\ 1 & -5.482462 & 4.093483 & -0.067857 \\ 1 & -4.227256 & 2.789751 & -0.106930 \\ 1 & -3.567022 & 4.558718 & -1.623284 \\ 1 & -6.447125 & 4.333801 & -2.660178 \\ 1 & -5.824865 & 6.182017 & -1.564567 \\ 1 & -4.170451 & 5.254949 & -4.449817 \\ 1 & -5.774048 & 4.513761 & -4.891811 \\ 1 & -5.018903 & 2.346540 & -4.379557 \\ 1 & -3.413210 & 3.072349 & -4.769384 \\ 8 & -6.629043 & 0.374418 & 3.489539 \\ 8 & -9.480984 & -0.669135 & 1.384743 \\ 11 & -8.857470 & 0.2730590 & 3.280850 \\ 8 & -9.052270 & 2.649440 & 2.650940 \\ 8 & -9.907771 & 1.440171 & 4.876169 \\ 1 & -6.390499 & 1.147911 & 2.958822 \\ 1 & -5.896771 & -0.216251 & 3.605130 \\ 1 & -8.945584 & -0.228495 & 0.695647 \\ 1 & -10.114720 & -1.277290 & 1.031899 \\ 1 & -9.681921 & 2.881698 & 1.978589 \\ 1 & -8.139679 & 2.720143 & 2.316400 \\ 1 & -9.705943 & 2.218594 & 4.347959 \\ 1 & -10.116790 & 1.6197060 & 5.781460 \\ 7 & 5.945743 & -0.771994 & -0.379407 \\ 6 & 6.508300 & -1.510115 & -1.460228 \\ 7 & 5.628899 & -2.419281 & -1.888787 \\ 6 & 4.470831 & -2.312056 & -1.102688 \\ 8 & 3.222710 & -3.034566 & -1.139161 \\ 8 & 2.850859 & -3.932783 & -1.893621\end{array}$




$\begin{array}{lccc}7 & 2.282720 & -2.625426 & -0.131581 \\ 6 & 2.568928 & -1.586891 & 0.780286 \\ 7 & 1.653456 & -1.330351 & 1.804232 \\ 7 & 3.733999 & -0.890798 & 0.796091 \\ 6 & 4.658191 & -1.287435 & -0.159052 \\ 6 & 11.972916 & 3.140276 & 0.075658 \\ 6 & 8.965980 & 0.980747 & -1.927425 \\ 6 & 8.420243 & 1.441998 & -0.557543 \\ 8 & 6.998510 & 1.318969 & -0.525680 \\ 6 & 9.020100 & 0.596066 & 0.605666 \\ 8 & 9.601360 & 1.435926 & 1.564759 \\ 6 & 7.809008 & -0.1476920 & 1.206950 \\ 6 & 6.587592 & 0.315869 & 0.392874 \\ 15 & 11.268190 & 1.382020 & 1.93342 \\ 8 & 11.19951 & 1.637637 & 3.5683840 \\ 8 & 12.01613 & 0.239256 & 1.4549580 \\ 8 & 11.74851 & 2.926193 & 1.4305200 \\ 8 & 8.625324 & 1.850092 & -2.969188 \\ 1 & 7.503503 & -1.3289700 & -1.886330 \\ 1 & 1.333106 & -3.0285400 & -0.145010 \\ 1 & 1.774064 & -0.4594320 & 2.266790 \\ 1 & 0.689808 & -1.5740940 & 1.621420 \\ 1 & 12.17295 & 4.211814 & -0.020149 \\ 1 & 11.09161 & 2.878339 & -0.526691 \\ 1 & 12.84231 & 2.573047 & -0.277828 \\ 1 & 10.07032 & 1.006285 & -1.931399 \\ 1 & 8.642574 & -0.0592090 & -2.159540 \\ 1 & 8.586996 & 2.536699 & -0.426030 \\ 1 & 9.784838 & -0.1240080 & 0.226900 \\ 1 & 7.665289 & 0.092211 & 2.278180 \\ 1 & 7.955611 & -1.2436830 & 1.166200 \\ 1 & 5.804120 & 0.802884 & 1.021014 \\ 1 & 10.68208 & 2.372894 & 3.8587470 \\ 1 & 7.677585 & 1.868658 & -3.026627\end{array}$

$\mathrm{Li}\left(\mathrm{H}_{2} \mathrm{O}\right)_{4}$-Inner sphere-monodentate- Anion Model 


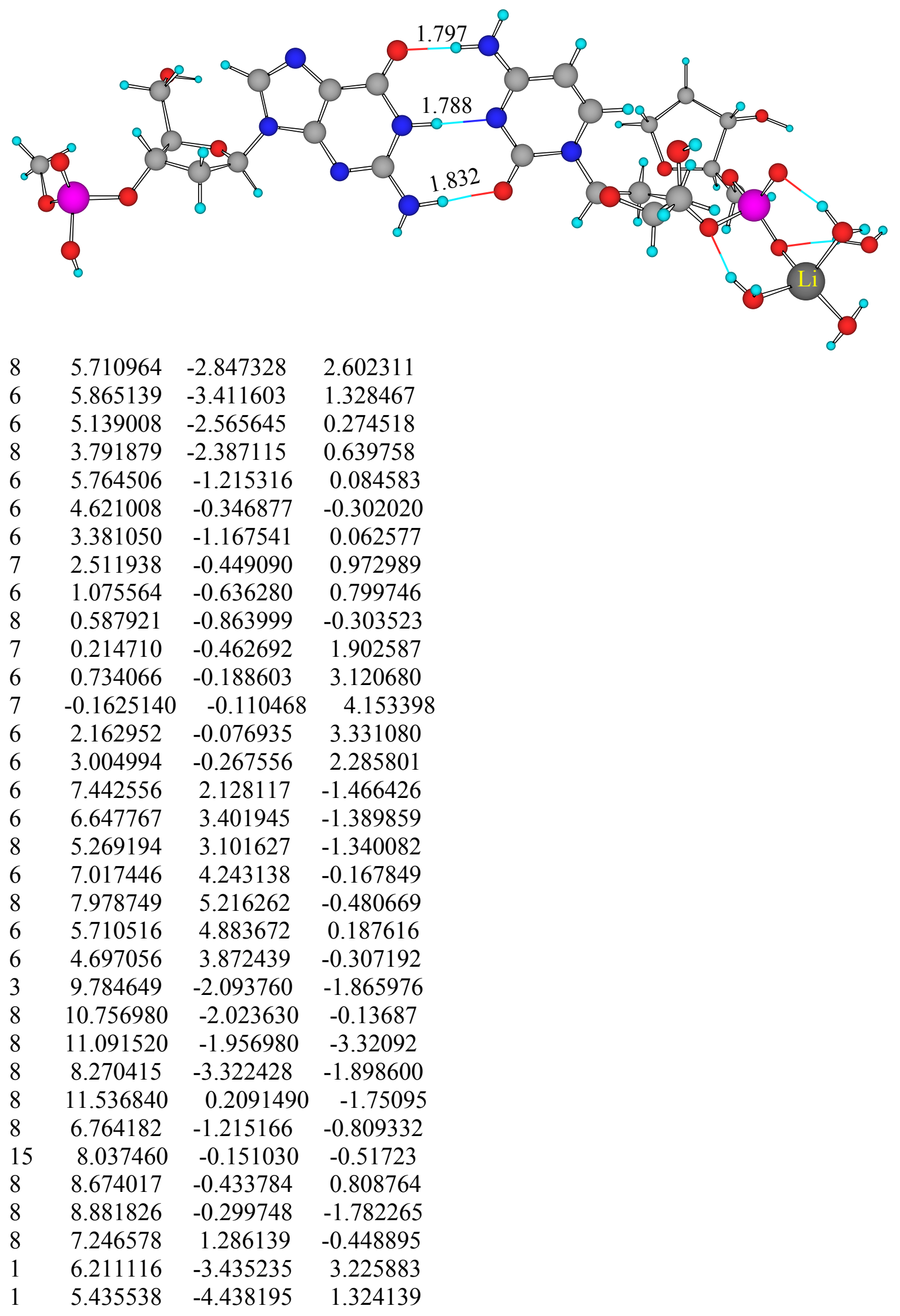




$\begin{array}{lccc}1 & 6.946244 & -3.481506 & 1.071178 \\ 1 & 5.165972 & -3.123289 & -0.690383 \\ 1 & 6.075839 & -0.846097 & 1.060502 \\ 1 & 4.634958 & -0.142339 & -1.396658 \\ 1 & 4.655204 & 0.632647 & 0.225490 \\ 1 & 2.852696 & -1.403231 & -0.888635 \\ 1 & 0.168373 & 0.286696 & 4.997559 \\ 1 & -1.1522950 & -0.027918 & 3.959530 \\ 1 & 2.552848 & 0.138985 & 4.332209 \\ 1 & 4.091447 & -0.214585 & 2.446176 \\ 1 & 8.492851 & 2.371433 & -1.596020 \\ 1 & 7.249144 & 1.658954 & -2.426634 \\ 1 & 6.833920 & 3.994694 & -2.314763 \\ 1 & 7.356820 & 3.613301 & 0.689005 \\ 1 & 8.848100 & 4.740937 & -0.539323 \\ 1 & 5.579564 & 5.843441 & -0.361210 \\ 1 & 5.618781 & 5.059109 & 1.2817050 \\ 1 & 4.402835 & 3.195538 & 0.5242280 \\ 1 & 3.786923 & 4.386483 & -0.6830410 \\ 1 & 10.09254 & -1.54383 & 0.418793 \\ 1 & 11.39680 & -1.32227 & -0.367469 \\ 1 & 10.66150 & -1.73844 & -4.162001 \\ 1 & 11.51281 & -1.12115 & -3.012264 \\ 1 & 8.338531 & -4.006877 & -1.2151580 \\ 1 & 7.550857 & -2.723357 & -1.5999870 \\ 1 & 10.54831 & 0.28682 & -1.715168 \\ 1 & 11.89837 & 1.10596 & -1.705164 \\ 7 & -6.519916 & 0.241469 & 0.6035700 \\ 6 & -6.936714 & 0.711292 & 1.8811670 \\ 7 & -5.897687 & 0.712247 & 2.7209000 \\ 6 & -4.773739 & 0.242123 & 2.0245260 \\ 6 & -3.406595 & 0.051054 & 2.4415440 \\ 8 & -2.870741 & 0.271283 & 3.5275860 \\ 7 & -2.553277 & -0.47813 & 1.4134720 \\ 6 & -3.029890 & -0.75012 & 0.1126120 \\ 7 & -2.162506 & -1.37853 & -0.793654 \\ 7 & -4.306733 & -0.54530 & -0.284341 \\ 6 & -5.151531 & -0.05271 & 0.7031890 \\ 6 & -13.24599 & 1.14905 & -1.835309 \\ 6 & -9.843999 & 2.112458 & 0.4572360 \\ 6 & -9.390072 & 1.247872 & -0.7399940 \\ 8 & -7.971339 & 1.317141 & -0.8901310 \\ 6 & -9.798390 & -0.24409 & -0.549237 \\ 8 & -10.52246 & -0.692400 & -1.660210 \\ 6 & -8.456861 & -1.00038 & -0.439863 \\ 6 & -7.361133 & 0.067347 & -0.6043080\end{array}$




$\begin{array}{llll}15 & -12.137490 & -1.24280 & -1.532490 \\ 8 & -12.09890 & -2.468060 & -2.645102 \\ 8 & -12.62054 & -1.541400 & -0.202227 \\ 8 & -12.93769 & -0.062300 & -2.443629 \\ 8 & -9.687830 & 3.483417 & 0.2286180 \\ 1 & -7.955688 & 1.044337 & 2.1202850 \\ 1 & -1.544278 & -0.56274 & 1.5999370 \\ 1 & -2.449583 & -1.32618 & -1.743715 \\ 1 & -1.177098 & -1.20557 & -0.654408 \\ 1 & -13.68447 & 1.76687 & -2.624827 \\ 1 & -12.34579 & 1.64266 & -1.442848 \\ 1 & -13.97513 & 1.01531 & -1.027295 \\ 1 & -10.93209 & 2.00809 & 0.617419 \\ 1 & -9.325312 & 1.806340 & 1.3939060 \\ 1 & -9.771138 & 1.681384 & -1.6940250 \\ 1 & -10.40920 & -0.378540 & 0.375892 \\ 1 & -8.353589 & -1.775473 & -1.223656 \\ 1 & -8.383046 & -1.541064 & 0.522729 \\ 1 & -6.685517 & -0.130248 & -1.470100 \\ 1 & -11.798580 & -2.24252 & -3.51201 \\ 1 & -8.762387 & 3.647088 & 0.093008\end{array}$

$\left.\underline{\mathrm{Na}\left(\mathrm{H}_{2}\right.} \underline{\mathrm{O}}\right)_{4} \underline{\text { - Inner sphere- monodentate- Anion Model }}$

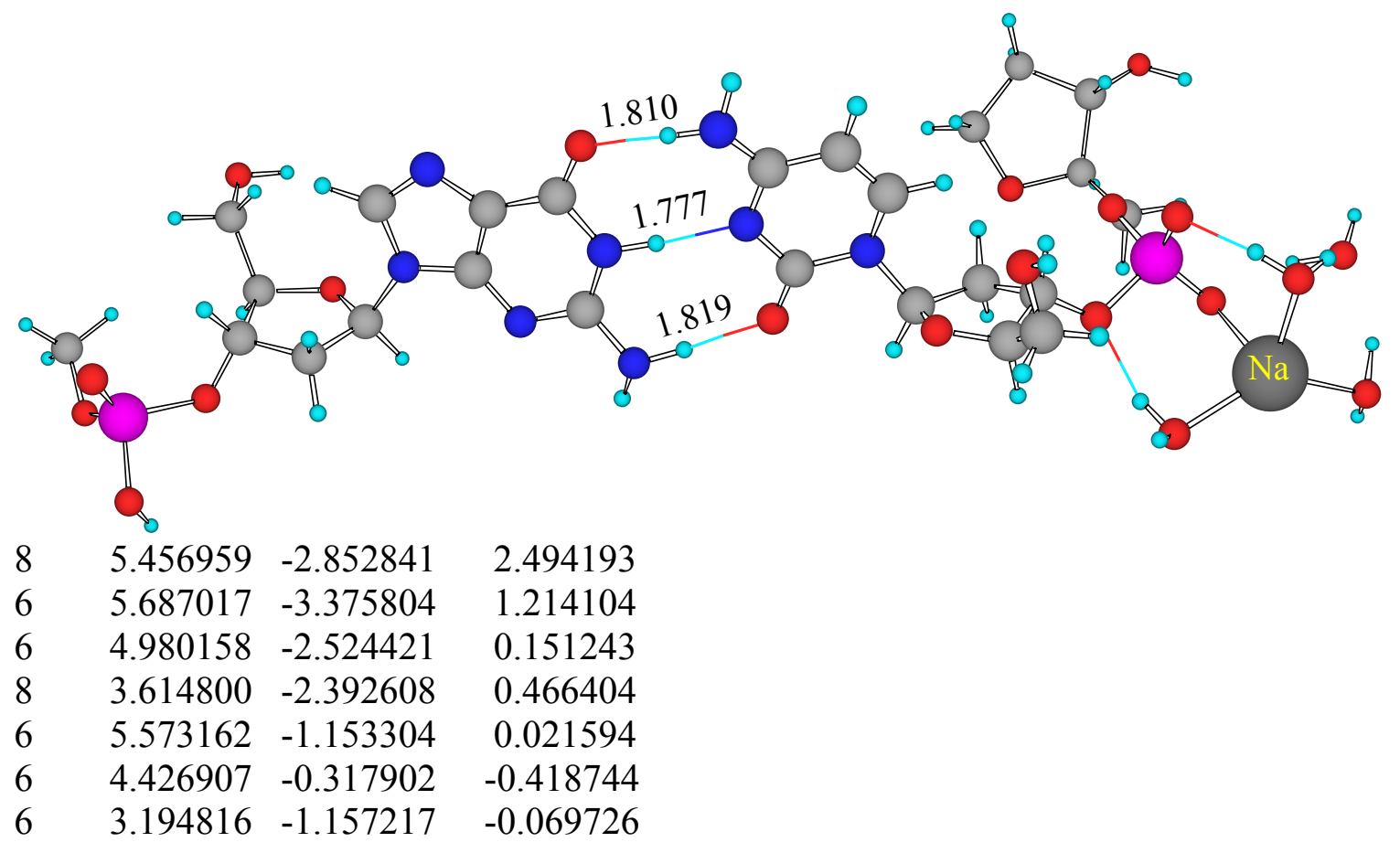




$\begin{array}{lccc}7 & 2.327319 & -0.476029 & 0.870734 \\ 6 & 0.895892 & -0.678168 & 0.721811 \\ 8 & 0.384532 & -0.939487 & -0.364862 \\ 7 & 0.046993 & -0.481650 & 1.833392 \\ 6 & 0.584234 & -0.189205 & 3.037735 \\ 7 & -0.297858 & -0.104761 & 4.092916 \\ 6 & 2.010966 & -0.071690 & 3.224399 \\ 6 & 2.841197 & -0.271428 & 2.167090 \\ 6 & 7.213659 & 2.226162 & -1.408749 \\ 6 & 6.372571 & 3.472327 & -1.391896 \\ 8 & 5.004432 & 3.124072 & -1.376172 \\ 6 & 6.675234 & 4.359224 & -0.183160 \\ 8 & 7.599016 & 5.367236 & -0.498788 \\ 6 & 5.333424 & 4.948544 & 0.128149 \\ 6 & 4.377665 & 3.883112 & -0.366824 \\ 11 & 9.92835 & -2.16665 & -1.60937 \\ 8 & 10.657917 & -1.78753 & 0.54401 \\ 8 & 11.678613 & -1.56091 & -2.96684 \\ 8 & 7.994085 & -3.31967 & -1.90763 \\ 8 & 11.410844 & 0.44415 & -1.13975 \\ 8 & 6.626130 & -1.10044 & -0.80779 \\ 15 & 7.850113 & -0.01133 & -0.38970 \\ 8 & 8.351389 & -0.29083 & 0.99362 \\ 8 & 8.806060 & -0.11934 & -1.57156 \\ 8 & 7.016566 & 1.405913 & -0.37361 \\ 1 & 5.950758 & -3.43964 & 3.12385 \\ 1 & 5.292943 & -4.41531 & 1.16285 \\ 1 & 6.780319 & -3.40315 & 1.00555 \\ 1 & 5.060345 & -3.05531 & -0.82541 \\ 1 & 5.810141 & -0.79555 & 1.02152 \\ 1 & 4.470994 & -0.14456 & -1.51806 \\ 1 & 4.424658 & 0.675409 & 0.082727 \\ 1 & 2.658675 & -1.36396 & -1.02312 \\ 1 & 0.047128 & 0.349021 & 4.903350 \\ 1 & -1.284292 & 0.00982 & 3.90185 \\ 1 & 2.418385 & 0.155010 & 4.215960 \\ 1 & 3.929621 & -0.21013 & 2.31647 \\ 1 & 8.257962 & 2.505028 & -1.51217 \\ 1 & 7.068529 & 1.724355 & -2.36108 \\ 1 & 6.566661 & 4.045909 & -2.32711 \\ 1 & 7.023021 & 3.766750 & 0.696603 \\ 1 & 8.489048 & 4.929283 & -0.53263 \\ 1 & 5.173383 & 5.889598 & -0.44482 \\ 1 & 5.208019 & 5.142442 & 1.215808 \\ 1 & 4.100028 & 3.206514 & 0.470660 \\ 1 & 3.451718 & 4.347650 & -0.76756\end{array}$




$\begin{array}{llll}1 & 9.852646 & -1.34877 & 0.91366 \\ 1 & 11.220176 & -1.02457 & 0.30817 \\ 1 & 11.450628 & -1.26837 & -3.86197 \\ 1 & 11.841347 & -0.73130 & -2.45876 \\ 1 & 7.765886 & -4.05123 & -1.31517 \\ 1 & 7.378437 & -2.59436 & -1.66028 \\ 1 & 10.423618 & 0.45390 & -1.26505 \\ 1 & 11.686467 & 1.36012 & -0.98997 \\ 7 & -6.691317 & 0.25252 & 0.59178 \\ 6 & -7.092795 & 0.75933 & 1.86119 \\ 7 & -6.044565 & 0.77889 & 2.68853 \\ 6 & -4.930537 & 0.28053 & 1.99516 \\ 6 & -3.559288 & 0.09144 & 2.40321 \\ 8 & -3.012264 & 0.33888 & 3.47680 \\ 7 & -2.720251 & -0.47338 & 1.38130 \\ 6 & -3.213166 & -0.78088 & 0.09605 \\ 7 & -2.36493 & -1.440838 & -0.799254 \\ 7 & -4.49648 & -0.577685 & -0.294672 \\ 6 & -5.32321 & -0.049878 & 0.686903 \\ 6 & -13.4099 & 1.116981 & -1.850547 \\ 6 & -10.0143 & 2.120245 & 0.426221 \\ 6 & -9.57656 & 1.216274 & -0.747874 \\ 8 & -8.16089 & 1.280260 & -0.920347 \\ 6 & -9.98193 & -0.268261 & -0.502015 \\ 8 & -10.7230 & -0.75373 & -1.58732 \\ 6 & -8.63960 & -1.021482 & -0.389102 \\ 6 & -7.54527 & 0.040620 & -0.598901 \\ 15 & -12.34200 & -1.27420 & -1.425110 \\ 8 & -12.3268 & -2.55596 & -2.47395 \\ 8 & -12.8341 & -1.49847 & -0.08306 \\ 8 & -13.1333 & -0.13220 & -2.39357 \\ 8 & -9.85983 & 3.482869 & 0.149883 \\ 1 & -8.10625 & 1.106357 & 2.100727 \\ 1 & -1.70708 & -0.560885 & 1.559804 \\ 1 & -2.66159 & -1.420431 & -1.747258 \\ 1 & -1.37334 & -1.290522 & -0.672358 \\ 1 & -13.8353 & 1.702861 & -2.670986 \\ 1 & -12.4977 & 1.609454 & -1.485278 \\ 1 & -14.1405 & 1.044136 & -1.036012 \\ 1 & -11.1002 & 2.022613 & 0.604025 \\ 1 & -9.48332 & 1.844851 & 1.365469 \\ 1 & -9.97123 & 1.618011 & -1.710262 \\ 1 & -10.5797 & -0.37230 & 0.435363 \\ 1 & -8.54663 & -1.818995 & -1.151417 \\ 1 & -8.55237 & -1.534106 & 0.587603 \\ 1 & -6.87962 & -0.184246 & -1.465915\end{array}$


$1 \quad-11.9803 \quad-2.39025 \quad-3.33713$

$\begin{array}{llll}1 & -8.93554 & 3.639981 & -0.001113\end{array}$

\section{${\underline{\mathrm{K}}\left(\mathrm{H}_{2}\right.}_{2} \underline{\mathrm{O}}_{4} \underline{\text { - Inner sphere- monodentate - Anion Model }}$}

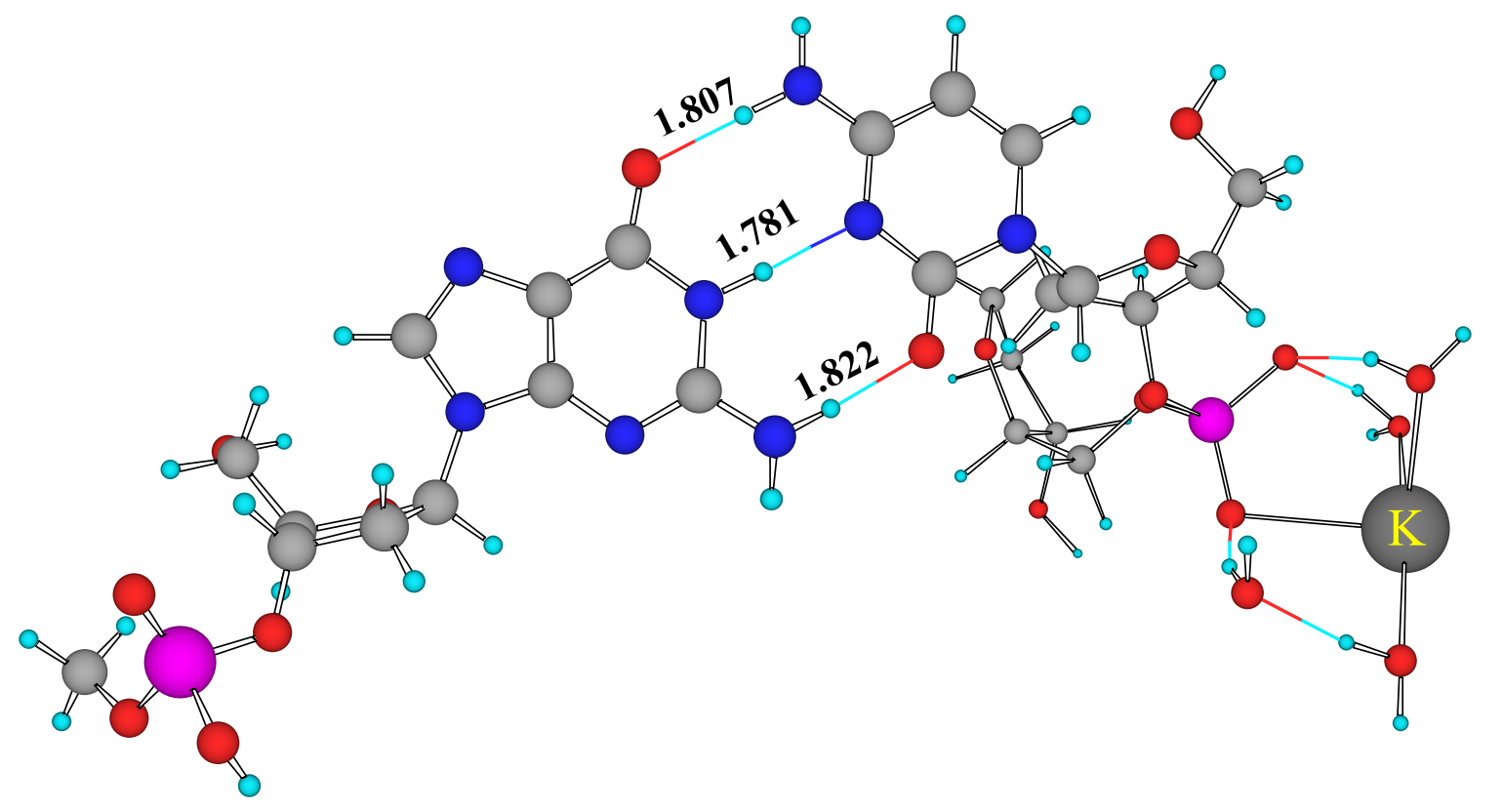

$\begin{array}{lccc}8 & -6.12517 & 3.42161 & 1.398899 \\ 6 & -6.77837 & 2.90067 & 0.274025 \\ 6 & -5.79085 & 2.13090 & -0.612292 \\ 8 & -4.78918 & 3.01206 & -1.077113 \\ 6 & -5.14126 & 0.94834 & 0.128481 \\ 8 & -5.19244 & -0.20164 & -0.66865 \\ 6 & -3.70754 & 1.38059 & 0.269478 \\ 6 & -3.54372 & 2.39117 & -0.854213 \\ 15 & -6.4400 & -1.21910 & -0.26077 \\ 8 & -7.71165 & -0.43714 & 0.023351 \\ 8 & -6.46525 & -2.25924 & -1.35877 \\ 7 & -2.52501 & 3.37399 & -0.550700 \\ 6 & -1.14001 & 2.94335 & -0.668182 \\ 8 & -0.80955 & 2.01930 & -1.403298 \\ 7 & -0.13698 & 3.64689 & 0.038818 \\ 6 & -0.48595 & 4.65928 & 0.858306 \\ 7 & 0.55224 & 5.36555 & 1.430694 \\ 6 & -1.86681 & 5.03068 & 1.065362 \\ 6 & -2.84434 & 4.35840 & 0.401784 \\ 8 & -6.00512 & -1.84945 & 1.197971 \\ 6 & -4.93522 & -2.73842 & 1.080608 \\ 6 & -4.45951 & -3.16232 & 2.474039\end{array}$




$\begin{array}{llll}8 & -4.09823 & -2.02283 & 3.225526 \\ 6 & -5.52205 & -3.95221 & 3.240084 \\ 8 & -5.33286 & -5.33648 & 3.109433 \\ 6 & -5.28995 & -3.51714 & 4.654811 \\ 6 & -4.72006 & -2.12428 & 4.486449 \\ 1 & -6.81770 & 3.90450 & 1.920236 \\ 1 & -7.224247 & 3.73135 & -0.317450 \\ 1 & -7.599077 & 2.22165 & 0.595500 \\ 1 & -6.356258 & 1.75855 & -1.495273 \\ 1 & -5.561266 & 0.78980 & 1.152026 \\ 1 & -2.994399 & 0.53277 & 0.167371 \\ 1 & -3.559281 & 1.86225 & 1.261667 \\ 1 & -3.275613 & 1.85057 & -1.791073 \\ 1 & 0.309110 & 5.90549 & 2.225490 \\ 1 & 1.474212 & 4.94886 & 1.445000 \\ 1 & -2.117866 & 5.83881 & 1.760702 \\ 1 & -3.895478 & 4.63050 & 0.569034 \\ 1 & -5.242421 & -3.64611 & 0.513904 \\ 1 & -4.073668 & -2.26029 & 0.559772 \\ 1 & -3.549217 & -3.79231 & 2.354159 \\ 1 & -6.557208 & -3.66560 & 2.937388 \\ 1 & -5.657658 & -5.57837 & 2.203285 \\ 1 & -4.537407 & -4.17115 & 5.150179 \\ 1 & -6.231403 & -3.50947 & 5.246304 \\ 1 & -5.536951 & -1.37218 & 4.544129 \\ 1 & -3.981753 & -1.90741 & 5.287488 \\ 8 & -5.667008 & -1.72586 & -3.901038 \\ 19 & -8.91669 & -2.11110 & -2.50745 \\ 8 & -9.829392 & -2.18801 & 0.052298 \\ 8 & -7.979046 & -2.73275 & -4.899336 \\ 8 & -8.999392 & 0.56570 & -2.157572 \\ 1 & -5.562964 & -0.76233 & -3.895148 \\ 1 & -5.769863 & -1.95998 & -2.944221 \\ 1 & -9.138335 & -1.51451 & 0.264241 \\ 1 & -9.722571 & -2.86508 & 0.735908 \\ 1 & -7.081104 & -2.35914 & -4.701117 \\ 1 & -7.816877 & -3.48644 & -5.483175 \\ 1 & -9.741121 & 1.14417 & -1.930240 \\ 1 & -8.523015 & 0.40942 & -1.305565 \\ 7 & 6.104029 & 0.74658 & 0.362554 \\ 6 & 6.664677 & 1.55738 & 1.390987 \\ 7 & 5.795405 & 2.51118 & 1.734951 \\ 6 & 4.646236 & 2.36174 & 0.942704 \\ 6 & 3.410188 & 3.10457 & 0.905638 \\ 8 & 3.043614 & 4.06371 & 1.583992 \\ 7 & 2.476942 & 2.63425 & -0.081110\end{array}$




$\begin{array}{lccc}6 & 2.757830 & 1.52429 & -0.906839 \\ 7 & 1.852437 & 1.20608 & -1.922796 \\ 7 & 3.910310 & 0.81012 & -0.851324 \\ 6 & 4.828587 & 1.26394 & 0.084154 \\ 6 & 12.073685 & -3.2753 & 0.313597 \\ 6 & 9.071001 & -0.92753 & 2.089220 \\ 6 & 8.53782 & -1.484995 & 0.750460 \\ 8 & 7.11881 & -1.343255 & 0.686316 \\ 6 & 9.16857 & -0.740969 & -0.464905 \\ 8 & 9.75157 & -1.661155 & -1.345941 \\ 6 & 7.97843 & -0.029204 & -1.142256 \\ 6 & 6.73793 & -0.408291 & -0.313418 \\ 15 & 11.42480 & -1.66050 & -1.68921 \\ 8 & 11.37800 & -2.04420 & -3.29967 \\ 8 & 12.18190 & -0.49358 & -1.29007 \\ 8 & 11.87370 & -3.16619 & -1.05751 \\ 8 & 8.69928 & -1.706975 & 3.189811 \\ 1 & 7.65085 & 1.393995 & 1.844462 \\ 1 & 1.53504 & 3.053302 & -0.114266 \\ 1 & 1.96378 & 0.300508 & -2.316029 \\ 1 & 0.89094 & 1.478700 & -1.773014 \\ 1 & 12.25990 & -4.33803 & 0.49645 \\ 1 & 11.18560 & -2.95803 & 0.87824 \\ 1 & 12.94350 & -2.69088 & 0.63648 \\ 1 & 10.17450 & -0.97121 & 2.11325 \\ 1 & 8.76307 & 0.132673 & 2.234539 \\ 1 & 8.68840 & -2.589025 & 0.706431 \\ 1 & 9.93829 & -0.004322 & -0.130664 \\ 1 & 7.84646 & -0.351535 & -2.193189 \\ 1 & 8.14208 & 1.064226 & -1.186358 \\ 1 & 5.95491 & -0.930089 & -0.913594 \\ 1 & 10.85570 & -2.79416 & -3.53941 \\ 1 & 7.75060 & -1.706018 & 3.232600\end{array}$

\section{$\underline{\mathrm{Li}\left(\mathrm{H}_{2}\right.} \underline{\mathrm{O}}_{4}{ }_{4}$ - Inner sphere- Bidentate- Anion Model}




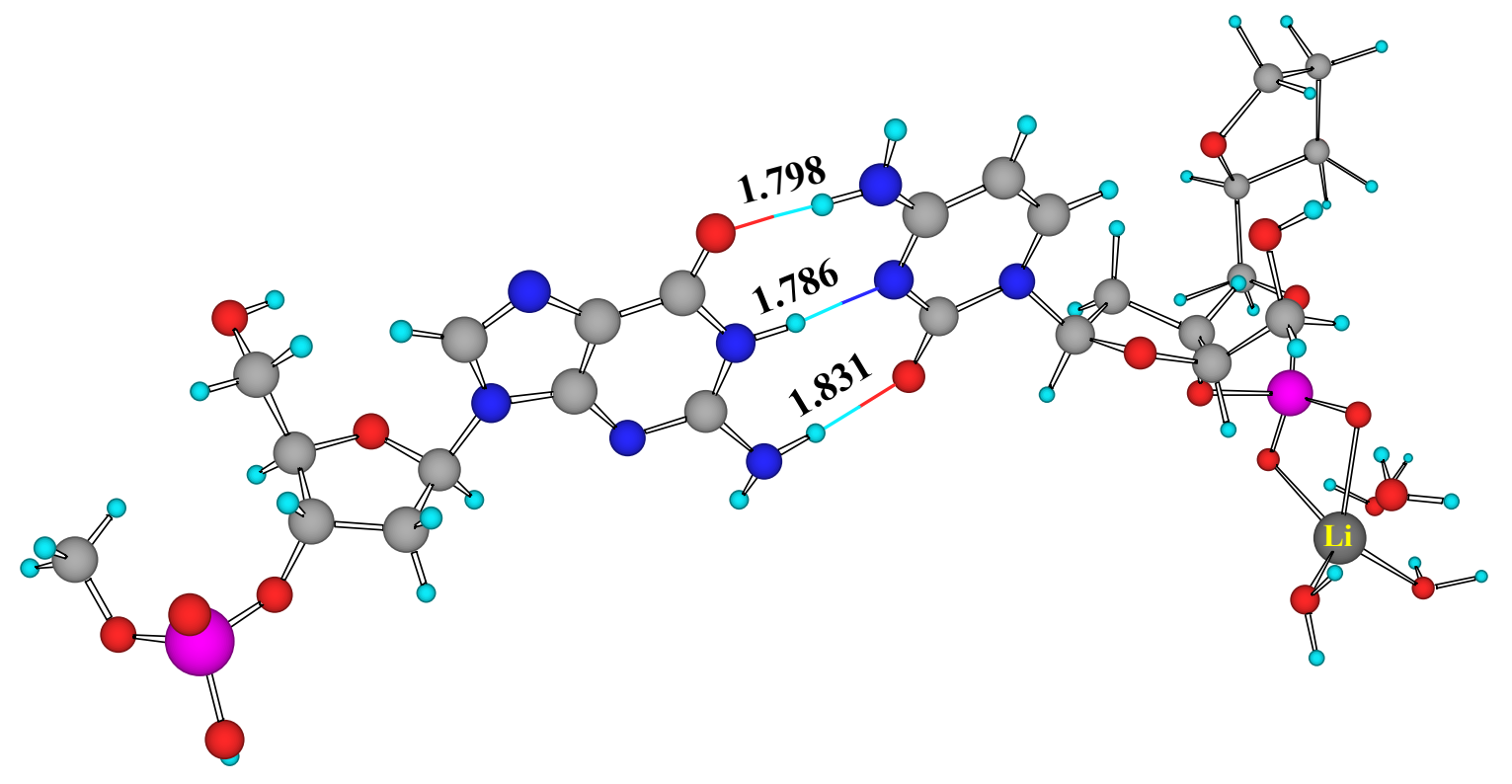

$\begin{array}{lccc}8 & 6.089261 & -2.162122 & -2.50623 \\ 6 & 6.218899 & -0.807223 & -2.83876 \\ 6 & 5.276398 & 0.049849 & -1.97671 \\ 8 & 3.949895 & -0.399693 & -2.15805 \\ 6 & 5.629314 & 0.026525 & -0.50112 \\ 6 & 4.462291 & -0.623222 & 0.14350 \\ 6 & 3.357455 & -0.451627 & -0.88309 \\ 7 & 2.386446 & -1.524853 & -0.80449 \\ 6 & 0.976346 & -1.158093 & -0.82660 \\ 8 & 0.585594 & -0.087911 & -0.37010 \\ 7 & 0.026519 & -2.088826 & -1.29609 \\ 6 & 0.437472 & -3.292436 & -1.75410 \\ 7 & -0.538033 & -4.105563 & -2.27162 \\ 6 & 1.837754 & -3.659990 & -1.77848 \\ 6 & 2.767053 & -2.761860 & -1.36546 \\ 6 & 7.689853 & 0.307978 & 2.42773 \\ 6 & 7.961712 & -0.996948 & 3.12229 \\ 8 & 7.313499 & -2.044705 & 2.43252 \\ 6 & 9.457006 & -1.307582 & 3.21464 \\ 8 & 9.989988 & -0.922709 & 4.45432 \\ 6 & 9.486636 & -2.798097 & 3.06494 \\ 6 & 8.255435 & -3.072774 & 2.22752 \\ 3 & 7.813960 & 3.802081 & -0.92793 \\ 8 & 6.565095 & 4.240267 & -2.30795 \\ 8 & 9.323385 & 4.995835 & -0.74606 \\ 8 & 5.869086 & 1.237209 & -0.00332 \\ 15 & 7.56227 & 1.641907 & 0.29341 \\ 8 & 8.166627 & 1.697859 & -1.09935 \\ 8 & 7.449465 & 2.972139 & 1.03227\end{array}$




\begin{tabular}{|c|c|c|c|}
\hline & 8.102286 & 0.361262 & 1.15652 \\
\hline & 6.715866 & -2.652638 & -3.09908 \\
\hline & 5.960254 & -0.660760 & -3.91133 \\
\hline & 7.271876 & -0.484907 & -2.68516 \\
\hline & 5.335514 & 1.095129 & -2.35526 \\
\hline & 6.472801 & -0.646041 & -0.37186 \\
\hline & 4.187674 & -0.136853 & 1.10637 \\
\hline & 4.676619 & -1.697051 & 0.34331 \\
\hline & 2.881148 & 0.537977 & -0.69511 \\
\hline & -0.292631 & -5.055026 & -2.40755 \\
\hline & -1.512684 & -3.904287 & -2.08804 \\
\hline & 2.137681 & -4.646057 & -2.15013 \\
\hline & 3.833655 & -3.019846 & -1.41176 \\
\hline & 8.120540 & 1.112020 & 3.01606 \\
\hline & 6.636127 & 0.548551 & 2.54410 \\
\hline & 7.531666 & -0.950145 & $45 \quad 4.14900$ \\
\hline & 10.03525 & -0.84833 & 2.377666 \\
\hline & 10.08820 & 0.06439 & 4.421306 \\
\hline & 9.38346 & -3.29623 & 4.055268 \\
\hline & 10.41464 & -3.14649 & 2.561326 \\
\hline & 8.52864 & -3.09785 & 1.149942 \\
\hline 1 & & -4.05176 & 2.502817 \\
\hline 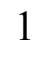 & 6.82832 & 3.603750 & -3.026970 \\
\hline & 6.56464 & 5.127459 & -2.695265 \\
\hline 1 & 10.12322 & 4.77726 & -1.246617 \\
\hline & 9.57262 & 4.896765 & 0.2190520 \\
\hline & 7.59312 & 2.216497 & -3.711961 \\
\hline & 7.80802 & 1.823765 & -2.831442 \\
\hline 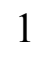 & 8.45167 & 2.334630 & -4.144572 \\
\hline & 9.61697 & 4.389748 & 1.8368200 \\
\hline . & 8.79052 & 3.847186 & 1.7460120 \\
\hline 1 & 10.31373 & 3.74116 & 2.019726 \\
\hline 7 & -6.61921 & -0.78652 & 0.006734 \\
\hline 6 & -7.15666 & -2.06174 & -0.327666 \\
\hline 7 & -6.18421 & -2.85207 & -0.790655 \\
\hline 6 & -4.98659 & -2.12069 & -0.782700 \\
\hline 6 & -3.64541 & -2.48506 & -1.167598 \\
\hline 8 & -3.20487 & -3.55322 & -1.592034 \\
\hline 7 & -2.68941 & -1.42205 & -1.022478 \\
\hline 6 & -3.05193 & -0.15335 & -0.520544 \\
\hline 7 & -2.08572 & 0.86348 & -0.526596 \\
\hline 7 & -4.30549 & 0.17817 & -0.134333 \\
\hline 6 & -5.24763 & -0.83189 & -0.286276 \\
\hline & -13.18517 & 1.37299 & 2.060688 \\
\hline 6 & -10.03964 & -1.38987 & 1.593314 \\
\hline & -9.44207 & 0.03447 & 1.561823 \\
\hline
\end{tabular}




$\begin{array}{llcc}8 & -8.02361 & -0.02078 & 1.719765 \\ 6 & -9.76583 & 0.75581 & 0.218828 \\ 8 & -10.37194 & 1.99259 & 0.469400 \\ 6 & -8.39018 & 0.95638 & -0.452892 \\ 6 & -7.35653 & 0.38610 & 0.534238 \\ 15 & -11.9528 & 2.35860 & -0.07283 \\ 8 & -11.74990 & 3.95711 & -0.452924 \\ 8 & -12.51670 & 1.48316 & -1.076646 \\ 8 & -12.75569 & 2.52280 & 1.408017 \\ 8 & -9.95739 & -1.98707 & 2.855399 \\ 1 & -8.20812 & -2.34913 & -0.193061 \\ 1 & -1.69415 & -1.62105 & -1.196434 \\ 1 & -2.30428 & 1.64488 & 0.047320 \\ 1 & -1.12454 & 0.56070 & -0.456197 \\ 1 & -13.59939 & 1.71748 & 3.013072 \\ 1 & -12.35067 & 0.68410 & 2.253273 \\ 1 & -13.96458 & 0.856805 & 1.487544 \\ 1 & -11.12738 & -1.355731 & 1.403367 \\ 1 & -9.57309 & -2.039007 & 0.818087 \\ 1 & -9.77789 & 0.612429 & 2.454279 \\ 1 & -10.43583 & 0.132288 & -0.420949 \\ 1 & -8.17797 & 2.024780 & -0.651015 \\ 1 & -8.35446 & 0.459343 & -1.440864 \\ 1 & -6.60778 & 1.143110 & 0.868175 \\ 1 & -11.39785 & 4.511627 & 0.226178 \\ 1 & -9.03728 & -2.057946 & 3.078951\end{array}$

$\left.\underline{\mathrm{Na}\left(\mathrm{H}_{2}\right.} \underline{\mathrm{O}}\right)_{4}$ - Inner sphere- Bidentate- Anion Model

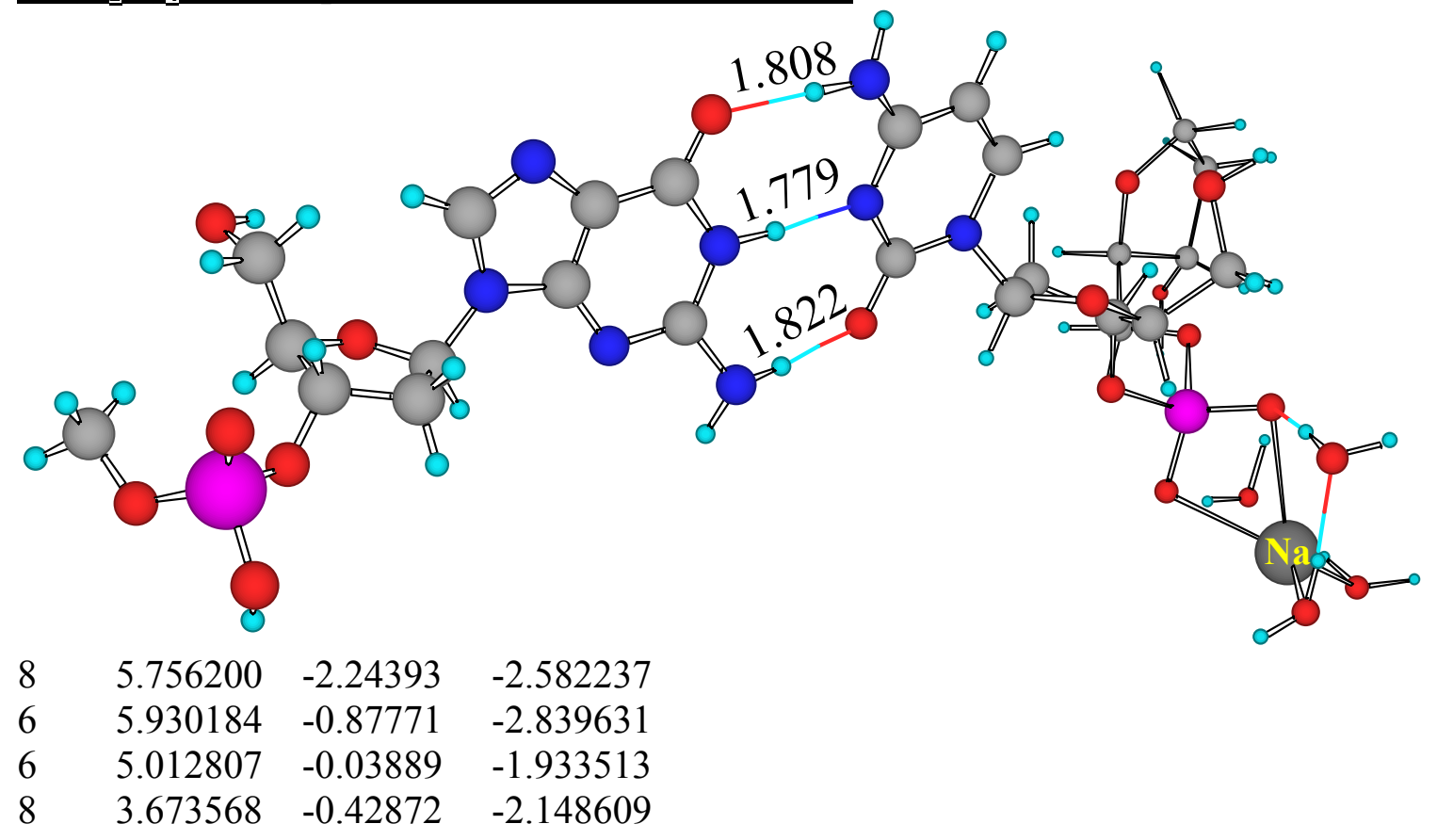




$\begin{array}{lccc}6 & 5.351194 & -0.15608 & -0.458297 \\ 6 & 4.139774 & -0.75238 & 0.155323 \\ 6 & 3.059721 & -0.51226 & -0.885564 \\ 7 & 2.061883 & -1.56300 & -0.869194 \\ 6 & 0.661469 & -1.17379 & -0.897417 \\ 8 & 0.272557 & -0.10322 & -0.441857 \\ 7 & -0.300228 & -2.093010 & -1.377217 \\ 6 & 0.097725 & -3.29414 & -1.844655 \\ 7 & -0.887350 & -4.093430 & -2.386378 \\ 6 & 1.489810 & -3.67900 & -1.865796 \\ 6 & 2.429919 & -2.79561 & -1.437320 \\ 6 & 7.506674 & 0.02767 & 2.336616 \\ 6 & 8.045895 & -1.16692 & 3.072384 \\ 8 & 7.720743 & -2.34695 & 2.370328 \\ 6 & 9.561357 & -1.10368 & 3.254408 \\ 8 & 9.912489 & -0.51967 & 4.481006 \\ 6 & 9.944314 & -2.551609 & 3.22581 \\ 6 & 8.869408 & -3.165208 & 2.35045 \\ 11 & 8.46650 & 3.734775 & -0.8749 \\ 8 & 7.489211 & 4.694796 & -2.66788 \\ 8 & 10.46342 & 4.317538 & -0.0487 \\ 8 & 5.682452 & 1.016313 & 0.10412 \\ 15 & 7.28358 & 1.396277 & 0.20876 \\ 8 & 7.978412 & 1.360161 & -1.13836 \\ 8 & 7.351643 & 2.719564 & 0.94559 \\ 8 & 7.968610 & 0.175699 & 1.08961 \\ 1 & 6.370348 & -2.720952 & -3.19867 \\ 1 & 5.678626 & -0.664841 & -3.90271 \\ 1 & 6.992477 & -0.597905 & -2.67003 \\ 1 & 5.110385 & 1.023401 & -2.25122 \\ 1 & 6.149327 & -0.890201 & -0.36087 \\ 1 & 3.872879 & -0.263132 & 1.11883 \\ 1 & 4.296071 & -1.836998 & 0.34953 \\ 1 & 2.602513 & 0.480251 & -0.66881 \\ 1 & -0.652963 & -5.051584 & -2.4809 \\ 1 & -1.854947 & -3.891411 & -2.1707 \\ 1 & 1.781062 & -4.663245 & -2.24790 \\ 1 & 3.493550 & -3.067996 & -1.48336 \\ 1 & 7.674849 & 0.915037 & 2.93767 \\ 1 & 6.422322 & -0.002725 & 2.37902 \\ 1 & 7.557328 & -1.219754 & 4.07258 \\ 1 & 10.06487 & -0.58073 & 2.4071 \\ 1 & 9.794213 & 0.459288 & 4.36807 \\ 1 & 9.892907 & -2.993055 & 4.24664 \\ 1 & 10.96037 & -2.70289 & 2.8002 \\ 1 & 9.234512 & -3.241724 & 1.30322\end{array}$




$\begin{array}{llll}1 & 8.610284 & -4.183869 & 2.70990 \\ 1 & 7.359515 & 3.860857 & -3.19555 \\ 1 & 6.616114 & 5.110328 & -2.61691 \\ 1 & 11.35762 & 4.205265 & -0.3990 \\ 1 & 10.43492 & 3.827012 & 0.81687 \\ 8 & 7.366419 & 2.199715 & -3.64029 \\ 1 & 7.508130 & 1.778432 & -2.75414 \\ 1 & 8.144774 & 1.945661 & -4.15845 \\ 8 & 9.857706 & 2.835614 & 2.11492 \\ 1 & 8.893038 & 2.868922 & 1.89939 \\ 1 & 10.08461 & 1.909651 & 1.93442 \\ 7 & -6.917948 & -0.760378 & 0.02052 \\ 6 & -7.468129 & -2.033679 & -0.3044 \\ 7 & -6.506648 & -2.830597 & -0.7778 \\ 6 & -5.303806 & -2.106985 & -0.7868 \\ 6 & -3.969386 & -2.479820 & -1.1887 \\ 8 & -3.54242 & -3.551469 & -1.617257 \\ 7 & -3.00496 & -1.422224 & -1.057902 \\ 6 & -3.35314 & -0.150397 & -0.555315 \\ 7 & -2.38659 & 0.858693 & -0.575829 \\ 7 & -4.60366 & 0.190069 & -0.152733 \\ 6 & -5.54965 & -0.815252 & -0.290255 \\ 6 & -13.4046 & 1.486616 & 2.201216 \\ 6 & -10.3212 & -1.33502 & 1.650866 \\ 6 & -9.71731 & 0.086269 & 1.601207 \\ 8 & -8.29839 & 0.025728 & 1.745778 \\ 6 & -10.0513 & 0.797314 & 0.255246 \\ 8 & -10.6532 & 2.038465 & 0.499747 \\ 6 & -8.68176 & 0.990982 & -0.429400 \\ 6 & -7.64048 & 0.418846 & 0.549265 \\ 15 & -12.24580 & 2.39450 & -0.005860 \\ 8 & -12.0502 & 3.978862 & -0.446112 \\ 8 & -12.8542 & 1.492607 & -0.959928 \\ 8 & -13.0047 & 2.613227 & 1.492256 \\ 8 & -10.2259 & -1.92259 & 2.916913 \\ 1 & -8.51881 & -2.314182 & -0.155144 \\ 1 & -2.01202 & -1.627402 & -1.248447 \\ 1 & -2.59350 & 1.650295 & -0.012362 \\ 1 & -1.42176 & 0.560775 & -0.536555 \\ 1 & -13.7766 & 1.862728 & 3.159070 \\ 1 & -12.5635 & 0.801913 & 2.379877 \\ 1 & -14.2090 & 0.953517 & 1.680394 \\ 1 & -11.4109 & -1.29757 & 1.473855 \\ 1 & -9.86653 & -1.992456 & 0.875593 \\ 1 & -10.0418 & 0.673318 & 2.491969 \\ 1 & -10.7285 & 0.170769 & -0.373789\end{array}$




$\begin{array}{cccc}1 & -8.46699 & 2.057993 & -0.632321 \\ 1 & -8.65671 & 0.491195 & -1.416285 \\ 1 & -6.88310 & 1.173007 & 0.870427 \\ 1 & -11.6254 & 4.542066 & 0.182358 \\ 1 & -9.30307 & -1.993146 & 3.129505\end{array}$

\section{$\mathrm{Na}\left(\mathrm{H}_{2} \mathrm{O}\right)_{4}$-Outer sphere- Dianion Model}

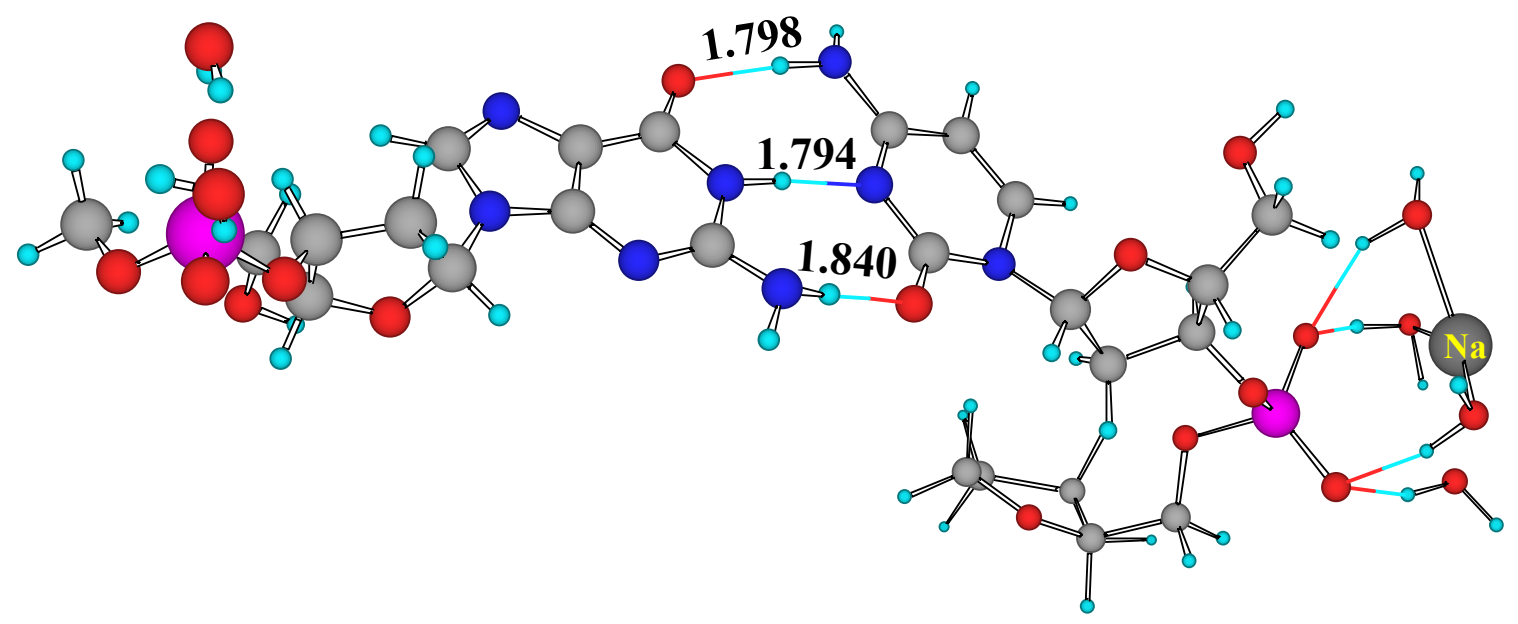

$\begin{array}{lccc}8 & 6.183101 & -3.438342 & 1.349961 \\ 6 & 6.322303 & -3.664420 & -0.026419 \\ 6 & 5.561333 & -2.599964 & -0.826997 \\ 8 & 4.211058 & -2.565506 & -0.431307 \\ 6 & 6.138605 & -1.226968 & -0.661093 \\ 8 & 7.159457 & -0.975525 & -1.492873 \\ 6 & 4.968368 & -0.330683 & -0.854312 \\ 6 & 3.757484 & -1.250353 & -0.668583 \\ 15 & 8.319120 & 0.067556 & -0.96328 \\ 8 & 8.907752 & -0.423320 & 0.351403 \\ 8 & 9.269579 & 0.222317 & -2.137702 \\ 7 & 2.910763 & -0.809920 & 0.422357 \\ 6 & 1.472745 & -0.978282 & 0.263791 \\ 8 & 0.937229 & -0.979122 & -0.840759 \\ 7 & 0.654763 & -1.048384 & 1.413378 \\ 6 & 1.220934 & -1.039133 & 2.637734 \\ 7 & 0.364730 & -1.201796 & 3.705628 \\ 6 & 2.653853 & -0.964297 & 2.812658 \\ 6 & 3.456542 & -0.911195 & 1.718480 \\ 8 & 7.479932 & 1.433907 & -0.620716 \\ 6 & 7.691798 & 2.483044 & -1.419294 \\ 6 & 6.846476 & 3.686670 & -1.108456 \\ 8 & 5.479583 & 3.343877 & -1.196942\end{array}$




$\begin{array}{lccc}6 & 7.130441 & 4.248567 & 0.285577 \\ 8 & 8.051997 & 5.305701 & 0.239815 \\ 6 & 5.781887 & 4.739028 & 0.716304 \\ 6 & 4.838128 & 3.825576 & -0.037562 \\ 1 & 6.724159 & -4.138169 & 1.799596 \\ 1 & 5.911656 & -4.667158 & -0.279446 \\ 1 & 7.398693 & -3.644036 & -0.310411 \\ 1 & 5.601487 & -2.887368 & -1.903502 \\ 1 & 6.413519 & -1.099373 & 0.384480 \\ 1 & 4.969303 & 0.095266 & -1.883324 \\ 1 & 4.975723 & 0.517747 & -0.133905 \\ 1 & 3.195244 & -1.251835 & -1.629293 \\ 1 & 0.732430 & -0.938077 & 4.587215 \\ 1 & -0.626065 & -1.040160 & 3.570076 \\ 1 & 3.084498 & -0.972351 & 3.819835 \\ 1 & 4.548146 & -0.882380 & 1.849123 \\ 1 & 8.737003 & 2.777117 & -1.435063 \\ 1 & 7.562443 & 2.231018 & -2.467853 \\ 1 & 7.050350 & 4.472707 & -1.871213 \\ 1 & 7.471720 & 3.458332 & 0.996332 \\ 1 & 8.945108 & 4.891868 & 0.112477 \\ 1 & 5.622602 & 5.791719 & 0.390865 \\ 1 & 5.643307 & 4.658624 & 1.816593 \\ 1 & 4.557564 & 2.960968 & 0.602683 \\ 1 & 3.912663 & 4.370975 & -0.320143 \\ 11 & 11.077900 & -1.509390 & -0.25128 \\ 8 & 11.43603 & 0.148970 & 1.417186 \\ 8 & 9.795885 & -2.857957 & 1.176583 \\ 1 & 10.46820 & 0.256390 & 1.296466 \\ 1 & 11.80728 & 0.688120 & 0.693846 \\ 1 & 10.08061 & -2.766080 & 2.09904 \\ 1 & 9.198215 & -2.087680 & 1.027689 \\ 8 & 10.40336 & -2.289320 & -2.32520 \\ 1 & 9.712505 & -2.969440 & -2.319542 \\ 1 & 9.922261 & -1.451284 & -2.527978 \\ 8 & 11.84131 & 0.492100 & -1.36691 \\ 1 & 10.92671 & 0.574810 & -1.74780 \\ 1 & 12.44310 & 0.504300 & -2.12570 \\ 7 & -6.069493 & 0.216312 & 0.590743 \\ 6 & -6.408216 & 0.455779 & 1.947927 \\ 7 & -5.346555 & 0.224354 & 2.730266 \\ 6 & -4.282507 & -0.170500 & 1.905857 \\ 6 & -2.919423 & -0.520090 & 2.216618 \\ 8 & -2.335369 & -0.551700 & 3.302175 \\ 6 & -2.134040 & -0.881100 & 1.071595 \\ & -2.666218 & -0.851060 & -0.23804\end{array}$




$\begin{array}{lccc}7 & -1.856603 & -1.325520 & -1.28870 \\ 7 & -3.935048 & -0.502470 & -0.53068 \\ 6 & -4.723748 & -0.172390 & 0.569248 \\ 6 & -8.974068 & 2.616219 & 0.969493 \\ 6 & -8.750342 & 1.897131 & -0.375967 \\ 8 & -7.348506 & 1.748988 & -0.626158 \\ 6 & -9.419274 & 0.481772 & -0.374179 \\ 6 & -8.221896 & -0.492260 & -0.53898 \\ 6 & -6.963926 & 0.386258 & -0.588493 \\ 8 & -8.500033 & 3.935118 & 0.993547 \\ 6 & -12.68337 & 2.0045140 & 0.438490 \\ 8 & -12.09806 & -0.9204170 & 0.095539 \\ 15 & -12.00250 & -0.080430 & -1.14786 \\ 8 & -10.31486 & 0.3613220 & -1.417915 \\ 8 & -12.54198 & -0.5244020 & -2.47561 \\ 8 & -12.64354 & 1.5128000 & -0.846927 \\ 1 & -7.39492 & 0.8059840 & 2.285082 \\ 1 & -1.12523 & -1.0451510 & 1.189378 \\ 1 & -2.16992 & -1.0571370 & -2.193354 \\ 1 & -0.86161 & -1.2050860 & -1.157650 \\ 1 & -10.06185 & 2.7261560 & 1.163783 \\ 1 & -8.52933 & 2.0389160 & 1.812323 \\ 1 & -9.09630 & 2.5336820 & -1.221424 \\ 1 & -9.93425 & 0.2972260 & 0.600682 \\ 1 & -8.30888 & -1.0832650 & -1.470781 \\ 1 & -8.19554 & -1.2330390 & 0.281523 \\ 1 & -6.35073 & 0.2295040 & -1.506285 \\ 1 & -7.58786 & 3.9107350 & 0.731362 \\ 1 & -13.25740 & 2.9381240 & 0.399312 \\ 1 & -11.67277 & 2.2371060 & 0.821153 \\ 1 & -13.17333 & 1.3166240 & 1.140018 \\ 8 & -13.47414 & -3.2963640 & 0.033757 \\ 1 & -13.53698 & -3.3736950 & -0.92447 \\ 8 & -13.73903 & -2.9360250 & -2.69496 \\ 1 & -12.96576 & -2.4923140 & 0.219921 \\ 1 & -14.57631 & -2.6486860 & -2.34548 \\ 1 & -13.16959 & -2.1484300 & -2.75753\end{array}$




\section{$\mathrm{K}\left(\mathrm{H}_{2} \mathrm{O}\right)_{4}$-Outer sphere- Dianion Model}

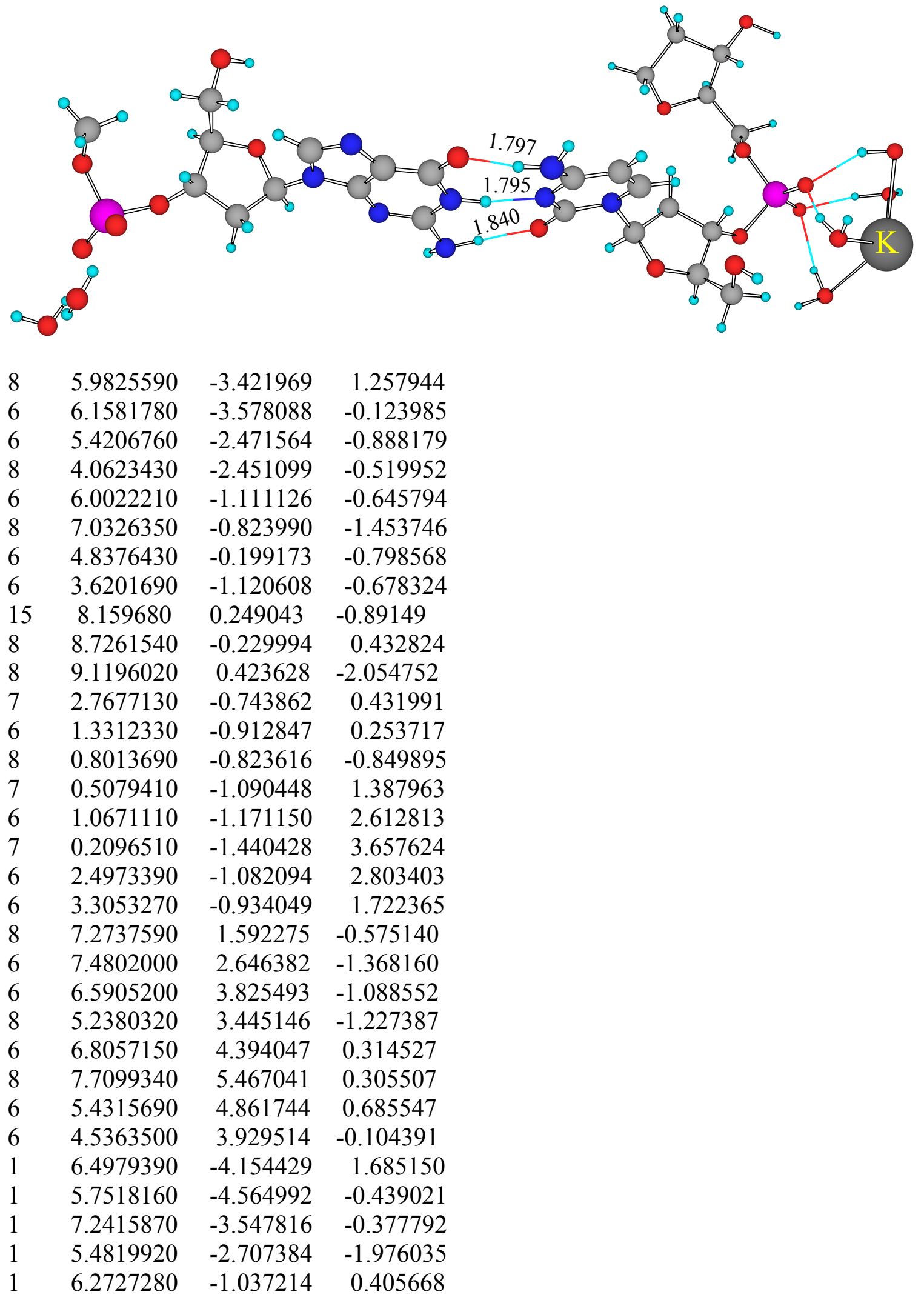




$\begin{array}{lccc}1 & 4.8513220 & 0.284993 & -1.801396 \\ 1 & 4.8402300 & 0.606394 & -0.030384 \\ 1 & 3.064947 & -1.058409 & -1.641108 \\ 1 & 0.566636 & -1.242824 & 4.560641 \\ 1 & -0.784117 & -1.292292 & 3.529025 \\ 1 & 2.921992 & -1.158108 & 3.810298 \\ 1 & 4.395486 & -0.899246 & 1.862987 \\ 1 & 8.516316 & 2.970812 & -1.349956 \\ 1 & 7.392729 & 2.390131 & -2.420037 \\ 1 & 6.800590 & 4.617806 & -1.843215 \\ 1 & 7.129683 & 3.610630 & 1.040690 \\ 1 & 8.614853 & 5.068647 & 0.218816 \\ 1 & 5.268057 & 5.910571 & 0.349719 \\ 1 & 5.247815 & 4.782548 & 1.779293 \\ 1 & 4.235957 & 3.067175 & 0.529733 \\ 1 & 3.618858 & 4.460600 & -0.436023 \\ 19 & 11.10090 & -1.875160 & -0.25519 \\ 8 & 11.34700 & 0.387847 & 1.308878 \\ 8 & 9.655169 & -2.273899 & -2.526193 \\ 8 & 9.194455 & -2.688954 & 1.498367 \\ 8 & 11.73895 & 0.569351 & -1.476490 \\ 1 & 10.37153 & 0.428707 & 1.227053 \\ 1 & 11.64914 & 0.813911 & 0.482953 \\ 1 & 8.766196 & -2.655755 & -2.460718 \\ 1 & 9.462891 & -1.314564 & -2.637359 \\ 1 & 9.279911 & -2.614503 & 2.460010 \\ 1 & 8.797026 & -1.830100 & 1.218522 \\ 1 & 10.78914 & 0.663463 & -1.758380 \\ 1 & 12.26145 & 1.026253 & -2.151078 \\ 7 & -6.229612 & 0.148880 & 0.641451 \\ 6 & -6.579315 & 0.256623 & 2.012721 \\ 7 & -5.518761 & -0.031165 & 2.777568 \\ 6 & -4.444614 & -0.333328 & 1.927451 \\ 6 & -3.078456 & -0.691138 & 2.214160 \\ 8 & -2.500326 & -0.813220 & 3.296518 \\ 7 & -2.281650 & -0.935268 & 1.046416 \\ 6 & -2.807178 & -0.793099 & -0.258583 \\ 7 & -1.985969 & -1.160287 & -1.342784 \\ 7 & -4.078726 & -0.434713 & -0.526688 \\ 6 & -4.878247 & -0.217440 & 0.593379 \\ 6 & -9.164375 & 2.469252 & 1.225008 \\ 6 & -8.922914 & 1.886680 & -0.182115 \\ 8 & -7.517881 & 1.776551 & -0.434067 \\ 6 & -9.577588 & 0.471696 & -0.323724 \\ 6 & -8.368246 & -0.471743 & -0.563095 \\ 6 & -7.119298 & 0.420098 & -0.522367\end{array}$




$\begin{array}{llll}8 & -8.704324 & 3.784057 & 1.381346 \\ 6 & -12.86177 & 1.84330 & 0.623008 \\ 8 & -12.238643 & -1.018611 & -0.044263 \\ 15 & -12.14393 & -0.04240 & -1.18245 \\ 8 & -10.458395 & 0.443236 & -1.386239 \\ 8 & -12.665837 & -0.334578 & -2.559097 \\ 8 & -12.804168 & 1.500813 & -0.709300 \\ 1 & -7.572759 & 0.5607590 & 2.3739040 \\ 1 & -1.272051 & -1.100108 & 1.1557680 \\ 1 & -2.299161 & -0.816111 & -2.221481 \\ 1 & -0.993396 & -1.038203 & -1.195926 \\ 1 & -10.254676 & 2.549328 & 1.419678 \\ 1 & -8.720211 & 1.8168540 & 2.0114850 \\ 1 & -9.268749 & 2.5993440 & -0.9644880 \\ 1 & -10.103658 & 0.190839 & 0.621793 \\ 1 & -8.440992 & -0.978955 & -1.544146 \\ 1 & -8.340925 & -1.281803 & 0.1891140 \\ 1 & -6.499833 & 0.3560200 & -1.4470540 \\ 1 & -7.789980 & 3.7942190 & 1.1258520 \\ 1 & -13.472472 & 2.751911 & 0.687561 \\ 1 & -11.860011 & 2.072007 & 1.030552 \\ 1 & -13.321687 & 1.062472 & 1.242815 \\ 8 & -13.652717 & -3.358633 & -0.374036 \\ 1 & -13.764311 & -3.313041 & -1.328584 \\ 1 & -13.134742 & -2.582644 & -0.112057 \\ 8 & -13.923624 & -2.676114 & -3.045097 \\ 1 & -14.796035 & -2.306016 & -3.125552 \\ 1 & -13.308462 & -1.928615 & -2.933571\end{array}$

$\underline{\mathrm{Li}\left(\mathrm{H}_{2}\right.} \underline{\mathrm{O}}_{4}{ }_{4}$ Inner sphere- monodentate - Dianion Model 


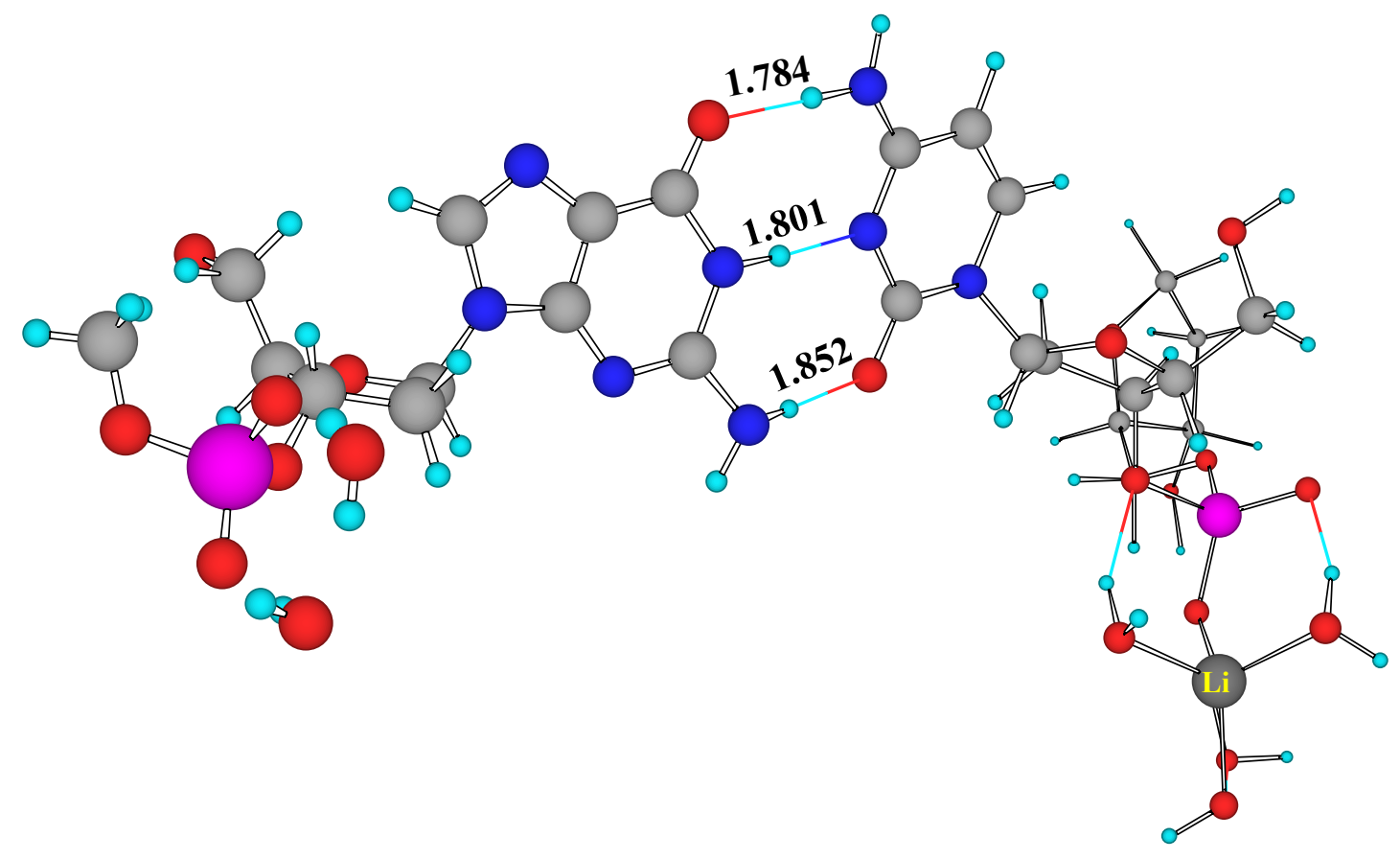

$\begin{array}{lccc}8 & 6.550046 & -2.198183 & -2.92760 \\ 6 & 6.603126 & -0.823556 & -3.19475 \\ 6 & 5.732767 & -0.048147 & -2.19271 \\ 8 & 4.400238 & -0.502645 & -2.29363 \\ 6 & 6.207793 & -0.170295 & -0.75524 \\ 6 & 5.087442 & -0.838236 & -0.04970 \\ 6 & 3.907945 & -0.621136 & -0.98133 \\ 7 & 2.944318 & -1.699825 & -0.88533 \\ 6 & 1.530780 & -1.328316 & -0.83915 \\ 8 & 1.167098 & -0.274056 & -0.33012 \\ 7 & 0.562830 & -2.239888 & -1.30607 \\ 6 & 0.949278 & -3.424722 & -1.82671 \\ 7 & -0.050925 & -4.216086 & -2.32624 \\ 6 & 2.348668 & -3.789794 & -1.93242 \\ 6 & 3.295790 & -2.909989 & -1.52528 \\ 6 & 8.572249 & 0.348363 & 1.98899 \\ 6 & 9.156336 & -0.783196 & 2.78757 \\ 8 & 8.730740 & -2.016271 & 2.24831 \\ 6 & 10.68483 & -0.750230 & 2.80870 \\ 8 & 11.17303 & -0.093560 & 3.94867 \\ 6 & 11.03608 & -2.206170 & 2.84419 \\ 6 & 9.861701 & -2.850812 & 2.13682 \\ 3 & 7.303495 & 4.212790 & -0.70039 \\ 8 & 7.896454 & 3.789499 & -2.53293 \\ 8 & 7.776291 & 5.947689 & 0.03314 \\ 8 & 5.410438 & 3.633424 & -0.62204 \\ 8 & 6.525690 & 1.010777 & -0.20543\end{array}$




$\begin{array}{lccc}15 & 8.14675 & 1.46361 & -0.239197 \\ 8 & 8.71520 & 1.36958 & -1.622753 \\ 8 & 8.08400 & 2.82329 & 0.4438700 \\ 8 & 8.89441 & 0.34308 & 0.6907560 \\ 1 & 7.12484 & -2.63358 & -3.609273 \\ 1 & 6.22576 & -0.63054 & -4.223939 \\ 1 & 7.65496 & -0.46802 & -3.138949 \\ 1 & 5.75098 & 1.02240 & -2.498993 \\ 1 & 7.05026 & -0.85970 & -0.740121 \\ 1 & 4.89055 & -0.38583 & 0.948037 \\ 1 & 5.30853 & -1.91902 & 0.095395 \\ 1 & 3.44838 & 0.35615 & -0.706794 \\ 1 & 0.18639 & -5.15832 & -2.514819 \\ 1 & -1.01849 & -4.01915 & -2.095059 \\ 1 & 2.62887 & -4.75864 & -2.360018 \\ 1 & 4.35829 & -3.16496 & -1.635752 \\ 1 & 8.83931 & 1.28610 & 2.4660090 \\ 1 & 7.49871 & 0.36995 & 2.1533830 \\ 1 & 8.77264 & -0.71799 & 3.831705 \\ 1 & 11.10851 & -0.297850 & 1.880366 \\ 1 & 11.05547 & 0.87794 & 3.782997 \\ 1 & 11.08789 & -2.573770 & 3.893834 \\ 1 & 11.99647 & -2.413710 & 2.323681 \\ 1 & 10.09927 & -2.988480 & 1.059444 \\ 1 & 9.63935 & -3.84478 & 2.580086 \\ 1 & 8.28313 & 2.88103 & -2.390290 \\ 1 & 8.61686 & 4.33419 & -2.883088 \\ 1 & 8.45420 & 5.67921 & 0.7147220 \\ 1 & 7.05169 & 6.35435 & 0.5325460 \\ 1 & 5.02315 & 3.57507 & -1.509365 \\ 1 & 5.55225 & 2.70331 & -0.345259 \\ 8 & 9.51512 & 4.73890 & 1.6919800 \\ 1 & 10.40338 & 4.72832 & 1.303897 \\ 1 & 9.10551 & 3.89040 & 1.3907080 \\ 8 & -12.304670 & 2.426180 & -3.121572 \\ 1 & -12.231560 & 3.311610 & -2.749133 \\ 1 & -12.069920 & 1.802690 & -2.417955 \\ 8 & -12.307420 & 4.717410 & -1.574309 \\ 1 & -13.231620 & 4.498110 & -1.632268 \\ 1 & -11.965420 & 4.306830 & -0.760346 \\ 7 & -6.03021 & -1.02297 & 0.327831 \\ 6 & -6.56895 & -2.29272 & -0.001062 \\ 7 & -5.61606 & -3.06782 & -0.536016 \\ 6 & -4.42822 & -2.32379 & -0.573951 \\ 6 & -3.10775 & -2.66448 & -1.036250 \\ 8 & -2.67644 & -3.72055 & -1.507622 \\ & & & \end{array}$




$\begin{array}{lrrr}7 & -2.156021 & -1.59873 & -0.91831 \\ 6 & -2.500314 & -0.34575 & -0.35740 \\ 7 & -1.528672 & 0.67940 & -0.38466 \\ 7 & -3.726538 & -0.03724 & 0.09850 \\ 6 & -4.678325 & -1.04820 & -0.03246 \\ 6 & -9.236723 & -1.80906 & 2.23295 \\ 6 & -8.719106 & -0.35939 & 2.15184 \\ 8 & -7.286596 & -0.34871 & 2.17853 \\ 6 & -9.209163 & 0.34100 & 0.84005 \\ 6 & -7.894327 & 0.67179 & 0.08388 \\ 6 & -6.756830 & 0.11988 & 0.95273 \\ 8 & -8.949115 & -2.44784 & 3.44695 \\ 6 & -12.71711 & -0.41570 & 1.56825 \\ 8 & -11.64590 & 1.03956 & -0.84305 \\ 15 & -11.54037 & 1.75990 & 0.47180 \\ 8 & -9.924595 & 1.48214 & 1.13586 \\ 8 & -11.80881 & 3.22677 & 0.62646 \\ 8 & -12.46594 & 0.93245 & 1.69340 \\ 1 & -7.612828 & -2.58776 & 0.18507 \\ 1 & -1.171721 & -1.78249 & -1.14086 \\ 1 & -1.730102 & 1.43764 & 0.22673 \\ 1 & -0.572485 & 0.36175 & -0.31867 \\ 1 & -10.34657 & -1.81720 & 2.18895 \\ 1 & -8.853165 & -2.42145 & 1.38494 \\ 1 & -8.998256 & 0.20707 & 3.06903 \\ 1 & -9.833139 & -0.36229 & 0.23509 \\ 1 & -7.772609 & 1.76358 & -0.05265 \\ 1 & -7.903006 & 0.24456 & -0.93593 \\ 1 & -6.000896 & 0.88995 & 1.23307 \\ 1 & -8.014222 & -2.36953 & 3.59210 \\ 1 & -13.46078 & -0.66820 & 2.33342 \\ 1 & -11.81062 & -1.02020 & 1.75619 \\ 1 & -13.11737 & -0.68360 & 0.58169\end{array}$

$\left.\underline{\mathrm{Na}\left(\mathrm{H}_{2}\right.} \underline{\underline{O}}\right)_{4}-$ Inner sphere- monodentate - Dianion Model

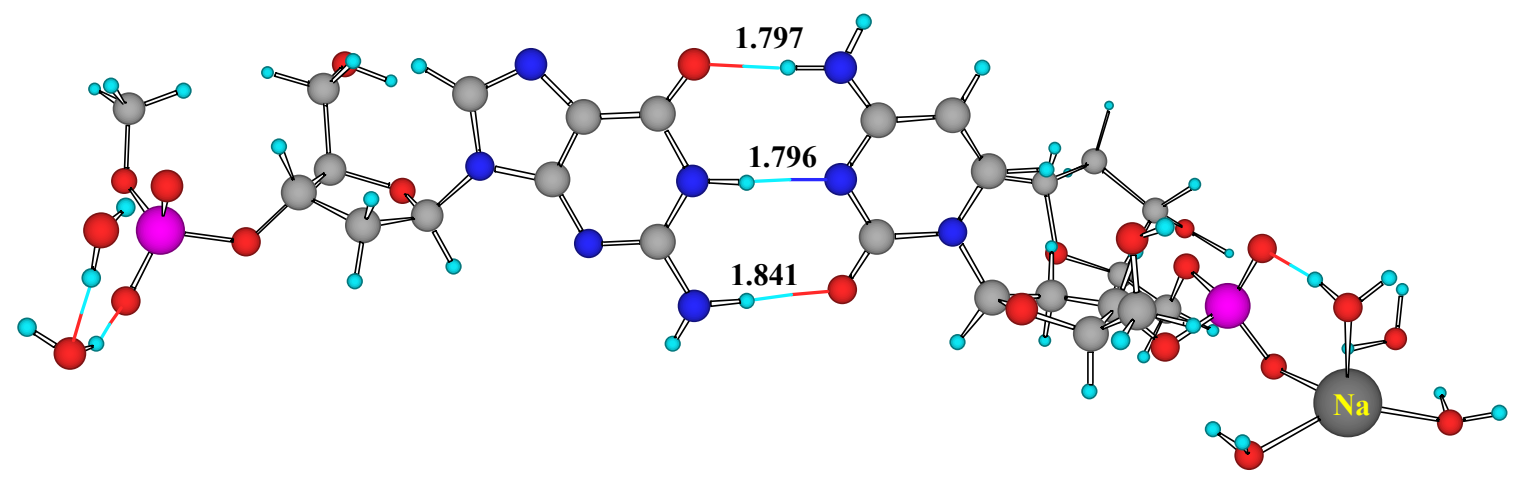




$\begin{array}{llll}8 & 6.0282420 & -3.362676 & 1.72442 \\ 6 & 6.3117030 & -3.559730 & 0.36584 \\ 6 & 5.6034660 & -2.500603 & -0.48979 \\ 8 & 4.2272230 & -2.482649 & -0.19296 \\ 6 & 6.1593870 & -1.123802 & -0.27916 \\ 6 & 4.9947370 & -0.233700 & -0.51543 \\ 6 & 3.7813950 & -1.160681 & -0.40298 \\ 7 & 2.8935880 & -0.757845 & 0.67024 \\ 6 & 1.4643260 & -0.948280 & 0.45648 \\ 8 & 0.9647400 & -0.891899 & -0.66307 \\ 7 & 0.6105400 & -1.107306 & 1.57130 \\ 6 & 1.1350030 & -1.148606 & 2.81297 \\ 7 & 0.2519450 & -1.404336 & 3.84045 \\ 6 & 2.5575030 & -1.032192 & 3.04193 \\ 6 & 3.3947750 & -0.901573 & 1.98111 \\ 6 & 7.5015080 & 2.328766 & -1.24083 \\ 6 & 6.6478880 & 3.550056 & -1.03699 \\ 8 & 5.3468340 & 3.163730 & -0.64847 \\ 6 & 7.2269410 & 4.495615 & 0.01804 \\ 8 & 7.9663570 & 5.534402 & -0.56779 \\ 6 & 5.9958370 & 5.031393 & 0.68333 \\ 6 & 5.0023300 & 3.904151 & 0.49980 \\ 11 & 10.7236600 & -1.723850 & -1.49680 \\ 8 & 10.380230 & -2.549070 & 0.6177 \\ 8 & 12.802040 & -0.939200 & -1.8622 \\ 8 & 8.9070070 & -2.786857 & -2.45487 \\ 8 & 7.2138970 & -0.850065 & -1.06188 \\ 15 & 8.409230 & 0.127185 & -0.3940 \\ 8 & 8.8230230 & -0.382779 & 0.95886 \\ 8 & 9.4353280 & 0.184784 & -1.51362 \\ 8 & 7.6525870 & 1.559873 & -0.15693 \\ 1 & 6.5253390 & -4.068078 & 2.21429 \\ 1 & 5.9571590 & -4.568154 & 0.05649 \\ 1 & 7.4103570 & -3.505492 & 0.19656 \\ 1 & 5.7240790 & -2.785637 & -1.56052 \\ 1 & 6.3822410 & -1.011233 & 0.77950 \\ 1 & 5.0418790 & 0.206919 & -1.53702 \\ 1 & 4.9633190 & 0.603389 & 0.21754 \\ 1 & 3.2538550 & -1.131072 & -1.38267 \\ 1 & 0.5807950 & -1.175304 & 4.74684 \\ 1 & -0.7390450 & -1.268801 & 3.68028 \\ 1 & 2.9534590 & -1.071776 & 4.06250 \\ 1 & 4.4789440 & -0.841598 & 2.15459 \\ 1 & 8.4593410 & 2.640996 & -1.64508 \\ 1 & 7.1213330 & 1.776479 & -2.09598 \\ 1 & 6.5636850 & 4.090120 & -2.00775\end{array}$




$\begin{array}{llll}1 & 7.841455 & 3.95513 & 0.77696 \\ 1 & 8.832564 & 5.13752 & -0.84592 \\ 1 & 5.628419 & 5.94203 & 0.15871 \\ 1 & 6.169403 & 5.25789 & 1.75795 \\ 1 & 5.028190 & 3.22844 & 1.38264 \\ 1 & 3.972891 & 4.30704 & 0.39179 \\ 1 & 9.767348 & -1.80932 & 0.88905 \\ 1 & 11.05655 & -2.57960 & 1.3096 \\ 1 & 12.62898 & 0.00260 & -1.5890 \\ 1 & 13.67390 & -1.16740 & -1.5121 \\ 1 & 8.641639 & -3.58506 & -1.9735 \\ 1 & 8.229291 & -2.12182 & -2.2136 \\ 8 & 11.80944 & 1.42530 & -1.0418 \\ 1 & 11.78295 & 1.48570 & -0.0741 \\ 1 & 10.88672 & 1.15070 & -1.2794 \\ 8 & -13.46938 & -3.31220 & -0.5044 \\ 1 & -13.50197 & -3.30040 & -1.4672 \\ 1 & -12.96669 & -2.53050 & -0.2289 \\ 8 & -13.64880 & -2.70020 & -3.1947 \\ 1 & -14.49730 & -2.44340 & -2.8485 \\ 1 & -13.07758 & -1.91200 & -3.1659 \\ 7 & -6.095837 & 0.15401 & 0.62363 \\ 6 & -6.481477 & 0.28525 & 1.98321 \\ 7 & -5.444875 & -0.00090 & 2.7807 \\ 6 & -4.350712 & -0.32430 & 1.9646 \\ 6 & -2.995689 & -0.68780 & 2.2937 \\ 8 & -2.448486 & -0.79670 & 3.3934 \\ 7 & -2.169379 & -0.95620 & 1.1520 \\ 6 & -2.658206 & -0.82870 & -0.1688 \\ 7 & -1.811107 & -1.21800 & -1.2246 \\ 7 & -3.919158 & -0.46470 & -0.4767 \\ 6 & -4.746965 & -0.22440 & 0.6176 \\ 6 & -9.016087 & 2.51677 & 1.09548 \\ 6 & -8.746380 & 1.90799 & -0.2952 \\ 8 & -7.336761 & 1.77921 & -0.5103 \\ 6 & -9.412357 & 0.49758 & -0.4294 \\ 6 & -8.208063 & -0.46130 & -0.6293 \\ 6 & -6.950819 & 0.41747 & -0.5677 \\ 8 & -8.546559 & 3.82944 & 1.24112 \\ 6 & -12.70444 & 1.92310 & 0.4173 \\ 8 & -12.09520 & -0.95650 & -0.1786 \\ 15 & -11.96494 & -0.00400 & -1.334 \\ 8 & -10.27114 & 0.46150 & -1.5097 \\ 8 & -12.46157 & -0.32230 & -2.7140 \\ 8 & -12.62121 & 1.55360 & -0.9063 \\ 1 & -7.481468 & 0.60329 & 2.31290\end{array}$




$\begin{array}{cccc}1 & -1.163329 & -1.120649 & 1.290782 \\ 1 & -2.097431 & -0.885218 & -2.116786 \\ 1 & -0.821834 & -1.102791 & -1.052673 \\ 1 & -10.10990 & 2.610748 & 1.262191 \\ 1 & -8.597975 & 1.873487 & 1.903450 \\ 1 & -9.065282 & 2.610747 & -1.097819 \\ 1 & -9.960663 & 0.236386 & 0.509077 \\ 1 & -8.262578 & -0.977781 & -1.606803 \\ 1 & -8.207727 & -1.263867 & 0.131331 \\ 1 & -6.307157 & 0.333196 & -1.474076 \\ 1 & -7.626461 & 3.826520 & 1.007006 \\ 1 & -13.29810 & 2.844666 & 0.449094 \\ 1 & -11.70884 & 2.139803 & 0.845950 \\ 1 & -13.19558 & 1.163746 & 1.039895\end{array}$

${\underline{\mathrm{K}}\left(\mathrm{H}_{2}\right.}_{2} \underline{\mathrm{O}}_{4}$ - Inner sphere- monodentate - Dianion Model

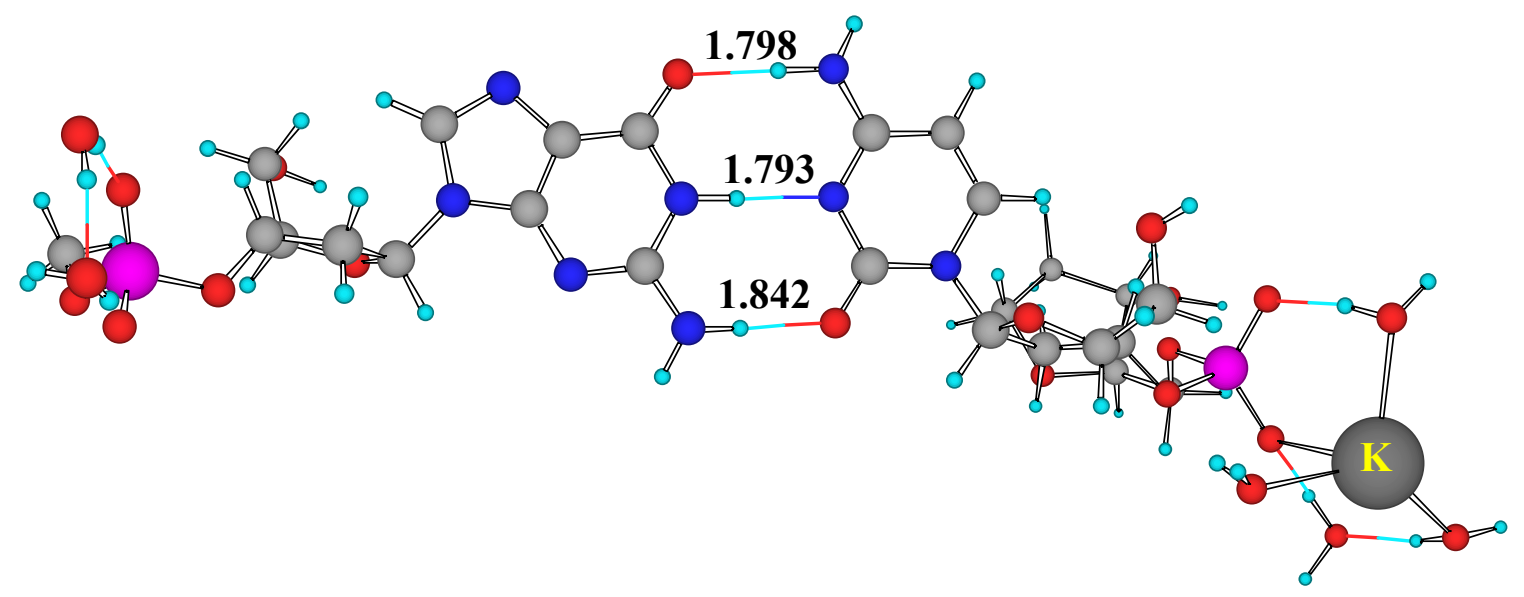

$\begin{array}{lllr}8 & 5.759310 & -3.0498040 & 2.261355 \\ 6 & 5.999728 & -3.4494570 & 0.939433 \\ 6 & 5.325572 & -2.4844600 & -0.044529 \\ 8 & 3.956425 & -2.3610470 & 0.257870 \\ 6 & 5.941243 & -1.1177780 & -0.026319 \\ 6 & 4.817653 & -0.2258790 & -0.412026 \\ 6 & 3.565200 & -1.0731640 & -0.165392 \\ 7 & 2.687999 & -0.4704880 & 0.819131 \\ 6 & 1.255231 & -0.6704700 & 0.638690 \\ 8 & 0.756159 & -0.8231610 & -0.472167 \\ 7 & 0.397993 & -0.5955280 & 1.759322 \\ 6 & 0.920824 & -0.4177850 & 2.989559 \\ 7 & 0.029762 & -0.4489170 & 4.041046 \\ 6 & 2.346172 & -0.3048770 & 3.200126 \\ 6 & 3.186069 & -0.3937700 & 2.136736 \\ 6 & 7.849618 & 2.394719 & -1.010345 \\ 6 & 7.061120 & 3.670878 & -0.913325\end{array}$




$\begin{array}{lccc}8 & 5.695681 & 3.407011 & -1.157214 \\ 6 & 7.202763 & 4.335555 & 0.456605 \\ 8 & 8.203209 & 5.319467 & 0.453942 \\ 6 & 5.852337 & 4.953083 & 0.655546 \\ 6 & 4.946601 & 4.039819 & -0.144090 \\ 19 & 10.79387 & -2.244440 & -1.38226 \\ 8 & 10.59721 & -2.307620 & 1.25129 \\ 8 & 12.75649 & -0.923420 & -2.59154 \\ 8 & 8.379831 & -3.170952 & -2.109740 \\ 8 & 7.015412 & -1.004591 & -0.821311 \\ 15 & 8.25476 & -0.000460 & -0.27161 \\ 8 & 8.675167 & -0.409159 & 1.111617 \\ 8 & 9.254413 & -0.060688 & -1.408919 \\ 8 & 7.456171 & 1.432551 & -0.173224 \\ 1 & 6.230633 & -3.706272 & 2.837152 \\ 1 & 5.587392 & -4.470814 & 0.780846 \\ 1 & 7.095530 & -3.477977 & 0.743881 \\ 1 & 5.417289 & -2.916368 & -1.067980 \\ 1 & 6.157132 & -0.861587 & 1.008600 \\ 1 & 4.888309 & 0.043528 & -1.490364 \\ 1 & 4.820615 & 0.719453 & 0.175258 \\ 1 & 3.044095 & -1.178547 & -1.143430 \\ 1 & 0.364160 & -0.063984 & 4.890706 \\ 1 & -0.957368 & -0.321960 & 3.852488 \\ 1 & 2.741910 & -0.172512 & 4.212909 \\ 1 & 4.271462 & -0.338791 & 2.304170 \\ 1 & 8.906693 & 2.619571 & -0.904869 \\ 1 & 7.841273 & 2.062919 & -2.044460 \\ 1 & 7.418701 & 4.372220 & -1.701756 \\ 1 & 7.383404 & 3.591041 & 1.268457 \\ 1 & 9.069746 & 4.836318 & 0.482813 \\ 1 & 5.821186 & 5.979800 & 0.226120 \\ 1 & 5.564412 & 4.983675 & 1.729069 \\ 1 & 4.513486 & 3.261247 & 0.520944 \\ 1 & 4.114790 & 4.618775 & -0.598729 \\ 1 & 9.862722 & -1.638322 & 1.310871 \\ 1 & 11.17770 & -2.097090 & 1.99588 \\ 1 & 13.62050 & -0.591480 & -2.31003 \\ 1 & 12.23541 & -0.111460 & -2.83080 \\ 1 & 7.892576 & -3.947393 & -1.798940 \\ 1 & 7.832204 & -2.409301 & -1.822656 \\ 8 & 11.03371 & 1.124260 & -3.04198 \\ 1 & 10.35051 & 0.856480 & -2.37591 \\ 1 & 10.58600 & 1.001470 & -3.89280 \\ 7 & -6.340700 & 0.296377 & 0.582314 \\ 6 & -6.731614 & 0.707926 & 1.883179\end{array}$




$\begin{array}{llll}7 & -5.685845 & 0.639221 & 2.716358 \\ 6 & -4.580943 & 0.181754 & 1.983141 \\ 6 & -3.215680 & -0.060868 & 2.375364 \\ 8 & -2.665955 & 0.084879 & 3.469570 \\ 7 & -2.380724 & -0.543634 & 1.313551 \\ 6 & -2.872559 & -0.725093 & 0.000491 \\ 7 & -2.012851 & -1.309583 & -0.94992 \\ 7 & -4.144450 & -0.476811 & -0.37130 \\ 6 & -4.979807 & -0.029205 & 0.649756 \\ 6 & -12.82278 & 2.18935 & -1.59417 \\ 6 & -9.366971 & 2.559115 & 0.530350 \\ 6 & -9.060001 & 1.690038 & -0.706662 \\ 8 & -7.644598 & 1.569496 & -0.882302 \\ 6 & -9.676000 & 0.258916 & -0.557507 \\ 8 & -10.44911 & -0.06577 & -1.65391 \\ 6 & -8.433127 & -0.667557 & -0.46477 \\ 6 & -7.209286 & 0.245546 & -0.626835 \\ 15 & -12.17637 & -0.424590 & -1.5271 \\ 8 & -12.45518 & -1.57108 & -2.45444 \\ 8 & -12.57420 & -0.44223 & -0.07608 \\ 8 & -12.77684 & 1.00984 & -2.30484 \\ 8 & -8.925639 & 3.884918 & 0.411864 \\ 1 & -7.742829 & 1.054525 & 2.142596 \\ 1 & -1.371881 & -0.655366 & 1.481848 \\ 1 & -2.306451 & -1.185265 & -1.89167 \\ 1 & -1.027291 & -1.133802 & -0.81054 \\ 1 & -13.25477 & 2.94135 & -2.26538 \\ 1 & -11.82048 & 2.52805 & -1.29057 \\ 1 & -13.45423 & 2.11209 & -0.69895 \\ 1 & -10.45848 & 2.66483 & 0.66460 \\ 1 & -8.948040 & 2.102206 & 1.456194 \\ 1 & -9.391163 & 2.205634 & -1.636904 \\ 1 & -10.27917 & 0.19225 & 0.38091 \\ 1 & -8.442783 & -1.437414 & -1.25986 \\ 1 & -8.425442 & -1.224754 & 0.490466 \\ 1 & -6.567506 & -0.025379 & -1.49732 \\ 1 & -8.000177 & 3.855866 & 0.201885 \\ 8 & -14.00320 & -2.55382 & 0.94247 \\ 8 & -13.70977 & -3.77412 & -1.52046 \\ 1 & -13.88343 & -3.15011 & 0.19533 \\ 1 & -13.51519 & -1.74196 & 0.73702 \\ 1 & -14.58487 & -3.40205 & -1.55882 \\ 1 & -13.10164 & -3.11495 & -1.90036\end{array}$

$\underline{\mathrm{Li}\left(\mathrm{H}_{2}\right.} \underline{\mathrm{O}}_{4}{ }_{4}$-Inner sphere- Bidentate- Dianion Model 


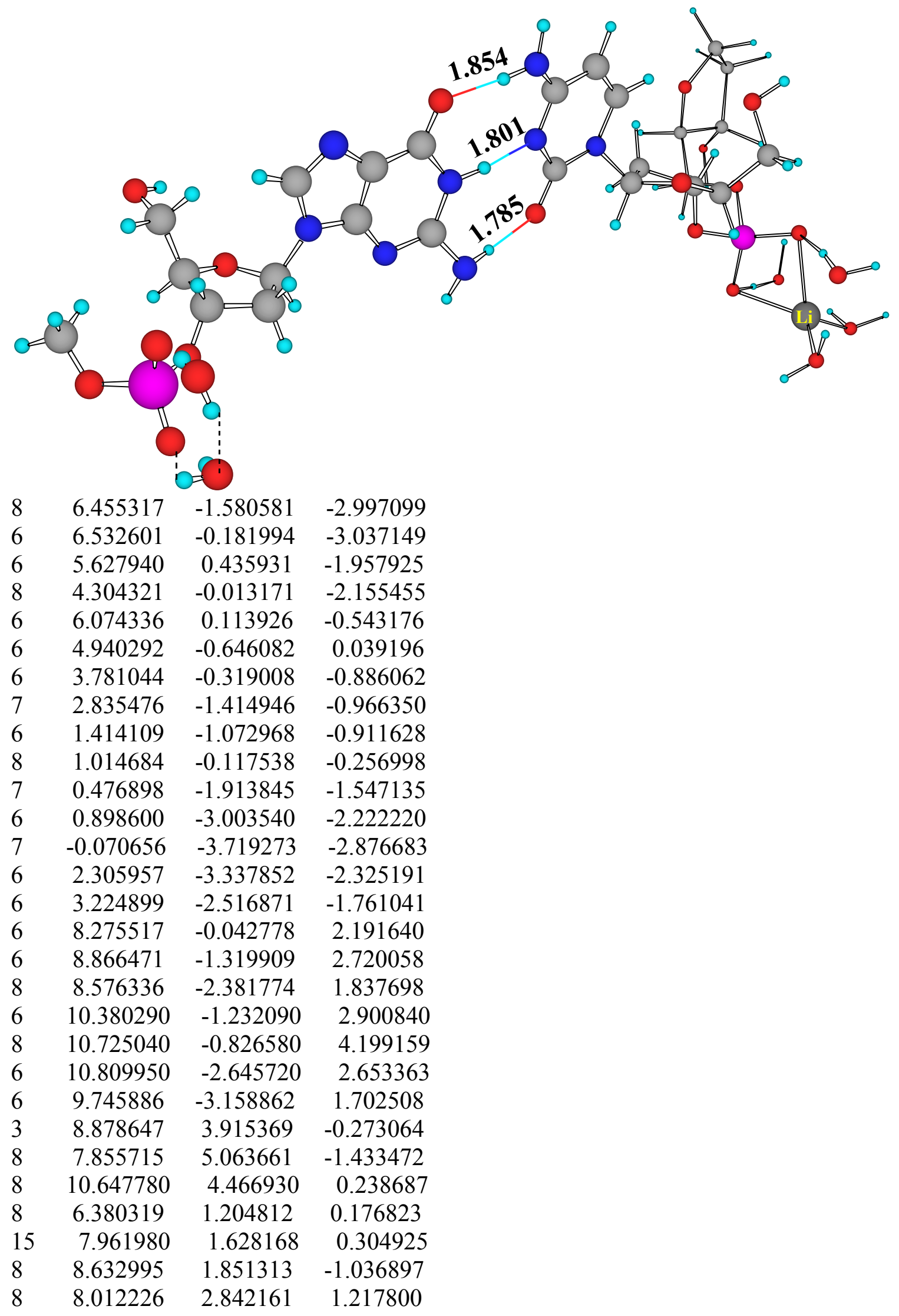




$\begin{array}{lccc}8 & 8.717427 & 0.325061 & 0.983119 \\ 1 & 7.056787 & -1.910403 & -3.71407 \\ 1 & 6.201284 & 0.180045 & -4.036079 \\ 1 & 7.585311 & 0.139766 & -2.880805 \\ 1 & 5.636344 & 1.539096 & -2.105503 \\ 1 & 6.913737 & -0.576545 & -0.61066 \\ 1 & 4.712979 & -0.328096 & 1.081369 \\ 1 & 5.167181 & -1.735517 & 0.051319 \\ 1 & 3.295752 & 0.603559 & -0.492574 \\ 1 & 0.186567 & -4.624821 & -3.18305 \\ 1 & -1.048230 & -3.56934 & -2.65204 \\ 1 & 2.614960 & -4.232732 & -2.87616 \\ 1 & 4.293892 & -2.743730 & -1.86772 \\ 1 & 8.419326 & 0.740926 & 2.927760 \\ 1 & 7.193900 & -0.120587 & 2.237265 \\ 1 & 8.392051 & -1.552403 & 3.701348 \\ 1 & 10.85657 & -0.57167 & 2.13819 \\ 1 & 10.57515 & 0.15391 & 4.23458 \\ 1 & 10.78492 & -3.23601 & 3.59703 \\ 1 & 11.82565 & -2.69683 & 2.20383 \\ 1 & 10.10745 & -3.07927 & 0.65433 \\ 1 & 9.514676 & -4.224149 & 1.916638 \\ 1 & 7.677667 & 4.484174 & -2.225877 \\ 1 & 6.987765 & 5.322271 & -1.088011 \\ 1 & 11.39974 & 4.34409 & -0.35795 \\ 1 & 10.82833 & 3.91445 & 1.04873 \\ 8 & 7.683946 & 3.116654 & -3.248087 \\ 1 & 7.990397 & 2.506877 & -2.530799 \\ 1 & 8.411071 & 3.133201 & -3.888331 \\ 8 & 10.53775 & 2.83233 & 2.35719 \\ 1 & 9.562265 & 2.863522 & 2.205575 \\ 1 & 10.77060 & 1.93472 & 2.07138 \\ 7 & -6.187265 & -1.03360 & 0.01798 \\ 6 & -6.692709 & -2.24623 & -0.51614 \\ 7 & -5.708968 & -2.92162 & -1.12508 \\ 6 & -4.533258 & -2.16659 & -1.00772 \\ 6 & -3.192374 & -2.41889 & -1.46788 \\ 8 & -2.728079 & -3.38685 & -2.07706 \\ 7 & -2.263108 & -1.37250 & -1.15712 \\ 6 & -2.647521 & -0.22068 & -0.42985 \\ 7 & -1.693224 & 0.807777 & -0.26976 \\ 7 & -3.894206 & 0.002358 & 0.021417 \\ 6 & -4.823533 & -0.98874 & -0.29233 \\ 6 & -12.663710 & 0.17199 & 2.68393 \\ 6 & -9.412085 & -2.11675 & 1.69350 \\ 6 & -8.913459 & -0.67080 & 1.89264\end{array}$




$\begin{array}{lccc}8 & -7.481992 & -0.646286 & 1.922243 \\ 6 & -9.410562 & 0.263326 & 0.739344 \\ 8 & -9.998925 & 1.401612 & 1.249092 \\ 6 & -8.110227 & 0.609936 & -0.036338 \\ 6 & -6.959872 & -0.005406 & 0.772294 \\ 15 & -11.66071 & 1.86978 & 0.85187 \\ 8 & -11.66560 & 3.363411 & 0.717581 \\ 8 & -12.17724 & 0.954715 & -0.22431 \\ 8 & -12.38893 & 1.488200 & 2.382571 \\ 8 & -9.094614 & -2.975290 & 2.755816 \\ 1 & -7.737511 & -2.576968 & -0.414733 \\ 1 & -1.269278 & -1.507601 & -1.372269 \\ 1 & -1.927868 & 1.465926 & 0.438033 \\ 1 & -0.734012 & 0.496506 & -0.219176 \\ 1 & -13.13625 & 0.170788 & 3.673416 \\ 1 & -11.74941 & -0.43918 & 2.73361 \\ 1 & -13.35395 & -0.28534 & 1.96259 \\ 1 & -10.51628 & -2.14301 & 1.66597 \\ 1 & -9.033084 & -2.545303 & 0.737456 \\ 1 & -9.198471 & -0.295604 & 2.901967 \\ 1 & -10.12343 & -0.28445 & 0.07556 \\ 1 & -7.972529 & 1.704208 & -0.127519 \\ 1 & -8.155810 & 0.226607 & -1.072817 \\ 1 & -6.236748 & 0.750858 & 1.157507 \\ 1 & -8.158284 & -2.912527 & 2.899478 \\ 8 & -13.11026 & 1.983425 & -2.59383 \\ 1 & -12.83208 & 2.894009 & -2.44728 \\ 1 & -12.82535 & 1.472560 & -1.82132 \\ 8 & -12.43902 & 4.482118 & -1.62025 \\ 1 & -13.37402 & 4.433515 & -1.44998 \\ 1 & -11.98438 & 4.174265 & -0.81613\end{array}$

\section{$\underline{\text { 5. Higher coordination geometries }}$}

\section{HPS $\bullet \mathrm{CSP}^{-} \bullet \bullet \mathbf{L i}\left(\mathrm{H}_{2} \mathrm{O}\right)_{5}$}

$\begin{array}{lrrr}8 & 5.924017000 & -3.057107000 & -2.141970000 \\ 6 & 6.077014000 & -1.760957000 & -2.651635000 \\ 6 & 5.136306000 & -0.787632000 & -1.930919000 \\ 8 & 3.797992000 & -1.197313000 & -2.124849000 \\ 6 & 5.436685000 & -0.693017000 & -0.422541000 \\ 8 & 5.539917000 & 0.649340000 & -0.035079000 \\ 6 & 4.204756000 & -1.285944000 & 0.209273000 \\ 6 & 3.155103000 & -1.114716000 & -0.873976000 \\ 15 & 7.103838000 & 1.170390000 & 0.105119000\end{array}$




$\begin{array}{lccc}8 & 7.937185000 & 0.741839000 & -1.087286000 \\ 8 & 6.973335000 & 2.650750000 & 0.427493000 \\ 7 & 2.109394000 & -2.111651000 & -0.761120000 \\ 6 & 0.726865000 & -1.656420000 & -0.815899000 \\ 8 & 0.406417000 & -0.527335000 & -0.458156000 \\ 7 & -0.280462000 & -2.563860000 & -1.204655000 \\ 6 & 0.053067000 & -3.824013000 & -1.562362000 \\ 7 & -0.975570000 & -4.616807000 & -2.003581000 \\ 6 & 1.428566000 & -4.276550000 & -1.565003000 \\ 6 & 2.413739000 & -3.407677000 & -1.224114000 \\ 8 & 7.727091000 & 0.336801000 & 1.381403000 \\ 6 & 7.139388000 & 0.716366000 & 2.589826000 \\ 6 & 7.660851000 & -0.174815000 & 3.721597000 \\ 8 & 7.405741000 & -1.530903000 & 3.422726000 \\ 6 & 9.158740000 & 0.010601000 & 3.968291000 \\ 8 & 9.403921000 & 0.926868000 & 5.002436000 \\ 6 & 9.592030000 & -1.366965000 & 4.367068000 \\ 6 & 8.596586000 & -2.251629000 & 3.645803000 \\ 8 & 6.587609000 & 4.091991000 & -1.790577000 \\ 3 & 8.228987000 & 3.394261000 & -2.721755000 \\ 8 & 9.737599000 & 2.797259000 & -1.671138000 \\ 8 & 8.526142000 & 5.247110000 & -3.369826000 \\ 8 & 7.548234000 & 1.754146000 & -3.549377000 \\ 8 & 9.291811000 & 4.078559000 & 0.687981000 \\ 7 & -6.818937000 & -0.767880000 & 0.052710000 \\ 6 & -7.432622000 & -2.035284000 & -0.156686000 \\ 7 & -6.514585000 & -2.918340000 & -0.559499000 \\ 6 & -5.277949000 & -2.259075000 & -0.633193000 \\ 6 & -3.966611000 & -2.732643000 & -1.001749000 \\ 8 & -3.594336000 & -3.857061000 & -1.336725000 \\ 7 & -2.949812000 & -1.717923000 & -0.963385000 \\ 6 & -3.230989000 & -0.391272000 & -0.570745000 \\ 7 & -2.207849000 & 0.562960000 & -0.676847000 \\ 7 & -4.457205000 & 0.045378000 & -0.202371000 \\ 6 & -5.457481000 & -0.918140000 & -0.251900000 \\ 6 & -13.219697000 & 1.934297000 & 1.992885000 \\ 6 & -10.228786000 & -1.031675000 & 1.747789000 \\ 6 & -9.560119000 & 0.349318000 & 1.566828000 \\ 8 & -8.143317000 & 0.234318000 & 1.707211000 \\ 6 & -9.874366000 & 0.952462000 & 0.164487000 \\ 8 & -10.414801000 & 2.236547000 & 0.301095000 \\ 6 & -8.503886000 & 1.017681000 & -0.543517000 \\ 6 & -7.481140000 & 0.488467000 & 0.477258000 \\ 8 & -11.986271000 & 2.626441000 & -0.252167000 \\ 8 & -12.614153000 & 1.681452000 & -1.149358000\end{array}$




$\begin{array}{cccc}8 & -12.748142000 & 2.985942000 & 1.215547000 \\ 8 & -10.149911000 & -1.506627000 & 3.060978000 \\ 1 & 6.557961000 & -3.628435000 & -2.648236000 \\ 1 & 5.838307000 & -1.756763000 & -3.738641000 \\ 1 & 7.130611000 & -1.426883000 & -2.524493000 \\ 1 & 5.262043000 & 0.211688000 & -2.404218000 \\ 1 & 6.318195000 & -1.307482000 & -0.115461000 \\ 1 & 3.913149000 & -0.764236000 & 1.147823000 \\ 1 & 4.381514000 & -2.360015000 & 0.436641000 \\ 1 & 2.742046000 & -0.084521000 & -0.776744000 \\ 1 & -1.970720000 & -1.988819000 & -1.130272000 \\ 1 & -0.789047000 & -5.587545000 & -2.059375000 \\ 1 & -1.933801000 & -4.342430000 & -1.827802000 \\ 1 & 1.665281000 & -5.304897000 & -1.859752000 \\ 1 & 3.462395000 & -3.733506000 & -1.251709000 \\ 1 & 7.382321000 & 1.776233000 & 2.829295000 \\ 1 & 6.032313000 & 0.598619000 & 2.547478000 \\ 1 & 7.101710000 & 0.079243000 & 4.650501000 \\ 1 & 9.702639000 & 0.308226000 & 3.040357000 \\ 1 & 9.256276000 & 1.829429000 & 4.616812000 \\ 1 & 9.490306000 & -1.509411000 & 5.466632000 \\ 1 & 10.637265000 & -1.580129000 & 4.053533000 \\ 1 & 9.013119000 & -2.566734000 & 2.664222000 \\ 1 & 8.378398000 & -3.160293000 & 4.246285000 \\ 1 & 5.784518000 & 3.752100000 & -2.215156000 \\ 1 & 6.619808000 & 3.638423000 & -0.900370000 \\ 1 & 9.363886000 & 1.927414000 & -1.401995000 \\ 1 & 9.771703000 & 3.302486000 & -0.818693000 \\ 1 & 7.812475000 & 5.543376000 & -2.774310000 \\ 1 & 9.344323000 & 5.614314000 & -2.999463000 \\ 1 & 8.148467000 & 1.304876000 & -4.163665000 \\ 1 & 7.596758000 & 1.228512000 & -2.703942000 \\ 1 & 8.419397000 & 3.615158000 & 0.749704000 \\ 1 & 9.789626000 & 3.782316000 & 1.464515000 \\ 1 & -8.495670000 & -2.248854000 & 0.018663000 \\ 1 & -2.373546000 & 1.403236000 & -0.172545000 \\ 1 & -1.265251000 & 0.211426000 & -0.585782000 \\ 1 & -13.593794000 & 2.397228000 & 2.911065000 \\ 1 & -12.416751000 & 1.226217000 & 2.241753000 \\ 1 & -14.037268000 & 1.403160000 & 1.491009000 \\ 1 & -11.317231000 & -0.958818000 & 1.573321000 \\ 1 & -9.812569000 & -1.776699000 & 1.032347000 \\ 1 & -9.847251000 & 1.028574000 & 2.403192000 \\ 1 & -8.242045000 & 2.050607000 & -0.843977000 \\ & -8.512700000 & 0.429936000 & -1.480999000\end{array}$




$\begin{array}{cccc}1 & -6.686111000 & 1.231455000 & 0.724283000 \\ 1 & -11.318991000 & 4.767470000 & -0.190125000 \\ 1 & -9.229959000 & -1.603615000 & 3.275231000\end{array}$

$\left(\mathrm{H}_{\mathbf{2}} \mathrm{O}\right)_{2} \boldsymbol{\bullet}^{\bullet}{ }^{-} \mathbf{P S G} \bullet \bullet \mathrm{CSP}^{-} \cdot \boldsymbol{\bullet} \mathbf{L i}\left(\mathrm{H}_{\mathbf{2}} \mathbf{O}\right)_{5}$

$\begin{array}{lccc}8 & 6.411831000 & -2.117071000 & -3.024602000 \\ 6 & 6.521645000 & -0.721960000 & -3.093816000 \\ 6 & 5.608497000 & -0.061013000 & -2.053979000 \\ 8 & 4.268177000 & -0.430388000 & -2.305073000 \\ 6 & 5.991808000 & -0.443821000 & -0.611425000 \\ 8 & 6.111254000 & 0.707564000 & 0.178360000 \\ 6 & 4.801343000 & -1.236360000 & -0.138180000 \\ 6 & 3.692520000 & -0.769319000 & -1.064790000 \\ 15 & 7.676709000 & 1.184250000 & 0.407846000 \\ 8 & 8.452341000 & 1.184927000 & -0.896060000 \\ 8 & 7.560494000 & 2.477054000 & 1.200768000 \\ 7 & 2.671991000 & -1.785755000 & -1.226879000 \\ 6 & 1.277846000 & -1.360013000 & -1.125513000 \\ 8 & 0.947699000 & -0.420205000 & -0.411875000 \\ 7 & 0.281660000 & -2.104495000 & -1.791154000 \\ 6 & 0.626285000 & -3.175355000 & -2.536578000 \\ 7 & -0.395379000 & -3.792087000 & -3.213923000 \\ 6 & 2.008248000 & -3.586639000 & -2.685940000 \\ 6 & 2.984409000 & -2.858944000 & -2.089027000 \\ 8 & 8.369930000 & -0.005468000 & 1.311851000 \\ 6 & 7.842965000 & -0.054587000 & 2.603927000 \\ 6 & 8.378185000 & -1.290185000 & 3.334676000 \\ 8 & 8.053215000 & -2.456269000 & 2.607910000 \\ 6 & 9.892218000 & -1.236095000 & 3.544742000 \\ 8 & 10.221434000 & -0.737196000 & 4.814280000 \\ 6 & 10.285838000 & -2.676580000 & 3.423012000 \\ 6 & 9.222410000 & -3.236407000 & 2.501442000 \\ 1 & 7.023982000 & -2.476059000 & -3.718132000 \\ 1 & 6.224919000 & -0.374288000 & -4.108544000 \\ 1 & 7.576323000 & -0.416744000 & -2.914713000 \\ 1 & 5.691706000 & 1.040698000 & -2.186612000 \\ 1 & 6.892158000 & -1.104206000 & -0.563536000 \\ 1 & 4.553476000 & -1.037602000 & 0.928233000 \\ 1 & 5.003503000 & -2.322858000 & -0.260136000 \\ 1 & 3.266838000 & 0.162355000 & -0.626386000 \\ 1 & -0.198210000 & -4.694385000 & -3.570731000 \\ 1 & -1.357120000 & -3.601927000 & -2.954478000 \\ 1 & 2.253985000 & -4.463251000 & -3.294975000 \\ 1 & 4.035953000 & -3.143787000 & -2.226353000 \\ 1 & 8.131931000 & 0.855270000 & 3.176672000 \\ 1 & 6.731467000 & -0.130689000 & 2.581805000\end{array}$




$\begin{array}{lccc}1 & 7.872361000 & -1.355208000 & 4.324604000 \\ 1 & 10.406029000 & -0.650580000 & 2.745700000 \\ 1 & 10.091438000 & 0.245780000 & 4.770269000 \\ 1 & 10.229316000 & -3.185787000 & 4.411485000 \\ 1 & 11.305702000 & -2.793368000 & 2.995770000 \\ 1 & 9.579352000 & -3.204029000 & 1.448884000 \\ 1 & 8.995153000 & -4.291000000 & 2.766124000 \\ 3 & 8.552433000 & 4.219439000 & -1.630567000 \\ 8 & 6.965657000 & 4.510688000 & -0.425812000 \\ 1 & 6.132080000 & 4.293129000 & -0.871536000 \\ 1 & 7.074650000 & 3.808037000 & 0.277159000 \\ 8 & 10.148991000 & 3.394017000 & -0.912989000 \\ 1 & 9.808521000 & 2.469830000 & -0.910017000 \\ 1 & 10.233850000 & 3.605083000 & 0.052002000 \\ 8 & 8.752746000 & 6.198679000 & -1.641621000 \\ 1 & 8.062393000 & 6.232876000 & -0.952497000 \\ 1 & 9.576365000 & 6.453935000 & -1.196780000 \\ 8 & 7.869543000 & 2.892486000 & -2.894573000 \\ 1 & 8.433665000 & 2.681437000 & -3.653843000 \\ 1 & 7.987204000 & 2.132217000 & -2.261258000 \\ 8 & 9.859535000 & 3.836867000 & 1.756913000 \\ 1 & 9.002382000 & 3.342058000 & 1.718596000 \\ 1 & 10.418304000 & 3.324243000 & 2.359966000 \\ 7 & -6.267269000 & -1.011202000 & 0.073253000 \\ 6 & -6.850629000 & -2.161752000 & -0.515789000 \\ 7 & -5.925039000 & -2.838262000 & -1.208918000 \\ 6 & -4.710958000 & -2.146178000 & -1.093581000 \\ 6 & -3.402271000 & -2.427752000 & -1.624490000 \\ 8 & -3.008485000 & -3.373145000 & -2.313228000 \\ 7 & -2.412732000 & -1.446622000 & -1.286422000 \\ 6 & -2.712807000 & -0.331246000 & -0.468619000 \\ 7 & -1.705013000 & 0.639797000 & -0.280104000 \\ 7 & -3.929144000 & -0.082456000 & 0.047645000 \\ 6 & -4.916201000 & -1.007267000 & -0.291394000 \\ 6 & -9.502015000 & -2.070153000 & 1.787648000 \\ 6 & -8.934857000 & -0.654348000 & 2.011187000 \\ 8 & -7.502814000 & -0.698526000 & 2.032033000 \\ 6 & -9.397899000 & 0.322692000 & 0.878771000 \\ 6 & -8.070774000 & 0.759429000 & 0.201812000 \\ 6 & -6.954601000 & -0.001498000 & 0.929071000 \\ 8 & -9.239035000 & -2.960075000 & 2.838079000 \\ 6 & -12.932062000 & -0.456760000 & 1.443738000 \\ 8 & -11.804722000 & 1.438866000 & -0.598774000 \\ 8 & -11.680866000 & 1.867128000 & 0.839804000 \\ 8 & -10.073000000 & 1.400921000 & 1.410824000 \\ & -1.895514000 & 3.269635000 & 1.307529000\end{array}$




$\begin{array}{cccc}8 & -12.632596000 & 0.822349000 & 1.856548000 \\ 1 & -7.904723000 & -2.452498000 & -0.390812000 \\ 1 & -1.434497000 & -1.616048000 & -1.544979000 \\ 1 & -1.881530000 & 1.257755000 & 0.479011000 \\ 1 & -0.760427000 & 0.282937000 & -0.288246000 \\ 1 & -10.611386000 & -2.031432000 & 1.748483000 \\ 1 & -9.137803000 & -2.502517000 & 0.827606000 \\ 1 & -9.196446000 & -0.284524000 & 3.028439000 \\ 1 & -10.044653000 & -0.216021000 & 0.143038000 \\ 1 & -7.910673000 & 1.850581000 & 0.297045000 \\ 1 & -8.092204000 & 0.556236000 & -0.884875000 \\ 1 & -6.173028000 & 0.666171000 & 1.360822000 \\ 1 & -8.302449000 & -2.945285000 & 2.992392000 \\ 1 & -13.682101000 & -0.843970000 & 2.143607000 \\ 1 & -12.047167000 & -1.118310000 & 1.486221000 \\ 1 & -13.344572000 & -0.489881000 & 0.426867000 \\ 8 & -12.447609000 & 2.935194000 & -2.765122000 \\ 1 & -12.527521000 & 3.750913000 & -2.276783000 \\ 1 & -12.209815000 & 2.248276000 & -2.122330000 \\ 8 & -12.541717000 & 5.445391000 & -0.180314000 \\ 1 & -12.569870000 & 4.962030000 & -1.002493000 \\ 1 & -12.311000000 & 4.805303000 & 0.510860000\end{array}$

\begin{tabular}{lccr}
\multicolumn{4}{l}{$\mathbf{H P S}^{\bullet \bullet} \mathbf{C S P}^{-} \bullet \mathbf{N a}\left(\mathbf{H}_{\mathbf{2}} \mathbf{O}\right)_{\mathbf{5}}$} \\
8 & 5.320601000 & -2.817120000 & 1.646098000 \\
6 & 5.510082000 & -3.233716000 & 0.321188000 \\
6 & 4.807124000 & -2.276681000 & -0.649360000 \\
8 & 3.449095000 & -2.156890000 & -0.298255000 \\
6 & 5.426541000 & -0.909248000 & -0.669631000 \\
6 & 4.280595000 & -0.007260000 & -0.957615000 \\
6 & 3.043204000 & -0.856943000 & -0.662503000 \\
7 & 2.229511000 & -0.278507000 & 0.388642000 \\
6 & 0.790447000 & -0.453993000 & 0.284211000 \\
8 & 0.227594000 & -0.581114000 & -0.801366000 \\
7 & -0.005847000 & -0.387043000 & 1.448474000 \\
6 & 0.588967000 & -0.245218000 & 2.653580000 \\
7 & -0.243062000 & -0.278877000 & 3.750117000 \\
6 & 2.024187000 & -0.162610000 & 2.787018000 \\
6 & 2.803301000 & -0.238833000 & 1.676542000 \\
6 & 6.960446000 & 2.689008000 & -1.663107000 \\
6 & 6.120041000 & 3.898202000 & -1.358384000 \\
8 & 4.752798000 & 3.548137000 & -1.381738000 \\
6 & 6.452279000 & 4.507558000 & 0.003549000 \\
8 & 7.389145000 & 5.546151000 & -0.108350000 \\
6 & 5.123685000 & 5.037505000 & 0.449382000 \\
6 & 4.144327000 & 4.109645000 & -0.240084000
\end{tabular}




$\begin{array}{lccc}8 & 6.436651000 & -0.791522000 & -1.545263000 \\ 15 & 7.645640000 & 0.303865000 & -1.156534000 \\ 8 & 8.308594000 & -0.142597000 & 0.132488000 \\ 8 & 8.489503000 & 0.410007000 & -2.400189000 \\ 8 & 6.796761000 & 1.667498000 & -0.818252000 \\ 1 & 5.811826000 & -3.467700000 & 2.211829000 \\ 1 & 5.086902000 & -4.254549000 & 0.190344000 \\ 1 & 6.594700000 & -3.271514000 & 0.086497000 \\ 1 & 4.863564000 & -2.719399000 & -1.670582000 \\ 1 & 5.729697000 & -0.658128000 & 0.345026000 \\ 1 & 4.285449000 & 0.299182000 & -2.028375000 \\ 1 & 4.316937000 & 0.917148000 & -0.340067000 \\ 1 & 2.465788000 & -0.933318000 & -1.611018000 \\ 1 & 0.146779000 & 0.059038000 & 4.595900000 \\ 1 & -1.235702000 & -0.134231000 & 3.623700000 \\ 1 & 2.479232000 & -0.063399000 & 3.779013000 \\ 1 & 3.898225000 & -0.206210000 & 1.783149000 \\ 1 & 8.000798000 & 2.990800000 & -1.739653000 \\ 1 & 6.782997000 & 2.398582000 & -2.694625000 \\ 1 & 6.289993000 & 4.659894000 & -2.153681000 \\ 1 & 6.799255000 & 3.738676000 & 0.734620000 \\ 1 & 8.272638000 & 5.112458000 & -0.236641000 \\ 1 & 4.972158000 & 6.078590000 & 0.084934000 \\ 1 & 5.014536000 & 5.005171000 & 1.555414000 \\ 1 & 3.852302000 & 3.289156000 & 0.450966000 \\ 1 & 3.228291000 & 4.663022000 & -0.537630000 \\ 7 & -6.827341000 & 0.226366000 & 0.646350000 \\ 6 & -7.176239000 & 0.566013000 & 1.985210000 \\ 7 & -6.084684000 & 0.538319000 & 2.754148000 \\ 6 & -4.993180000 & 0.171755000 & 1.951409000 \\ 6 & -3.595470000 & -0.004487000 & 2.264617000 \\ 8 & -3.000064000 & 0.139933000 & 3.331103000 \\ 7 & -2.793598000 & -0.410669000 & 1.142285000 \\ 6 & -3.346050000 & -0.593166000 & -0.142304000 \\ 7 & -2.526976000 & -1.113127000 & -1.149705000 \\ 7 & -4.655840000 & -0.401820000 & -0.441258000 \\ 6 & -5.445609000 & -0.024961000 & 0.635612000 \\ 6 & -13.716751000 & 0.983442000 & -1.310055000 \\ 6 & -10.240026000 & 1.920903000 & 0.874452000 \\ 6 & -9.830015000 & 1.176348000 & -0.415843000 \\ 8 & -8.431869000 & 1.335812000 & -0.656029000 \\ 6 & -10.148443000 & -0.346028000 & -0.317434000 \\ 8 & -10.933410000 & -0.747069000 & -1.406455000 \\ 6 & -8.767686000 & -1.033537000 & -0.368325000 \\ 6 & -7.739689000 & 0.103128000 & -0.512992000 \\ 15 & -12.509245000 & -1.376939000 & -1.210860000\end{array}$




$\begin{array}{lccc}8 & -12.493913000 & -2.535082000 & -2.394855000 \\ 8 & -12.903667000 & -1.773101000 & 0.123631000 \\ 8 & -13.418662000 & -0.185818000 & -1.999900000 \\ 8 & -10.167874000 & 3.312018000 & 0.745310000 \\ 1 & -8.187245000 & 0.837645000 & 2.315282000 \\ 1 & -1.769859000 & -0.479105000 & 1.260391000 \\ 1 & -2.875666000 & -0.996868000 & -2.072743000 \\ 1 & -1.534956000 & -0.942000000 & -1.056866000 \\ 1 & -14.217458000 & 1.630292000 & -2.036865000 \\ 1 & -12.806965000 & 1.480571000 & -0.944988000 \\ 1 & -14.392213000 & 0.787528000 & -0.468679000 \\ 1 & -11.307458000 & 1.745414000 & 1.098251000 \\ 1 & -9.641424000 & 1.573504000 & 1.746790000 \\ 1 & -10.300330000 & 1.659328000 & -1.304070000 \\ 1 & -10.680127000 & -0.585346000 & 0.634889000 \\ 1 & -8.684134000 & -1.732874000 & -1.222535000 \\ 1 & -8.596391000 & -1.648647000 & 0.535395000 \\ 1 & -7.116983000 & 0.013298000 & -1.434640000 \\ 1 & -12.214893000 & -2.256512000 & -3.253412000 \\ 1 & -9.262607000 & 3.534741000 & 0.564537000 \\ 11 & 10.217561000 & -1.347739000 & 0.003815000 \\ 8 & 8.439394000 & -1.855665000 & 2.295386000 \\ 8 & 10.520518000 & -1.199752000 & -2.287732000 \\ 8 & 8.325455000 & -3.006190000 & -2.253990000 \\ 8 & 11.042317000 & -1.110526000 & 2.121671000 \\ 8 & 9.344980000 & -3.500442000 & 0.150814000 \\ 1 & 8.137037000 & -1.174035000 & 1.653833000 \\ 1 & 8.545280000 & -2.648573000 & 1.731073000 \\ 1 & 11.245429000 & -1.046714000 & -2.910708000 \\ 1 & 9.839885000 & -0.469809000 & -2.441018000 \\ 1 & 7.575676000 & -2.381405000 & -2.193862000 \\ 1 & 9.077454000 & -2.457647000 & -2.561721000 \\ 1 & 10.133129000 & -1.373207000 & 2.433471000 \\ 1 & 11.185858000 & -0.225960000 & 2.489757000 \\ 1 & 9.822765000 & -4.342078000 & 0.172459000 \\ 1 & 8.877597000 & -3.460365000 & -0.735394000\end{array}$

\section{HPS $\bullet \mathrm{CSP}^{-} \boldsymbol{\bullet} \mathbf{N a}\left(\mathrm{H}_{\mathbf{2}} \mathrm{O}\right)_{\mathbf{6}}$}

$\begin{array}{lrrr}8 & 5.335713000 & -3.096218000 & 1.371028000 \\ 6 & 5.510982000 & -3.262249000 & -0.009678000 \\ 6 & 4.728285000 & -2.194974000 & -0.784716000 \\ 8 & 3.367580000 & -2.244061000 & -0.428895000 \\ 6 & 5.235909000 & -0.804050000 & -0.542750000 \\ 8 & 6.254495000 & -0.460840000 & -1.344871000 \\ 6 & 4.025469000 & 0.044540000 & -0.702508000 \\ 6 & 2.858147000 & -0.939609000 & -0.596794000\end{array}$




$\begin{array}{lccc}15 & 7.304847000 & 0.715330000 & -0.780180000 \\ 8 & 7.880493000 & 0.248507000 & 0.545700000 \\ 8 & 8.240035000 & 0.966546000 & -1.940454000 \\ 7 & 1.974789000 & -0.605578000 & 0.502511000 \\ 6 & 0.549316000 & -0.798504000 & 0.302592000 \\ 8 & 0.036143000 & -0.752258000 & -0.813385000 \\ 7 & -0.292154000 & -0.952841000 & 1.426013000 \\ 6 & 0.249751000 & -0.999493000 & 2.662963000 \\ 7 & -0.623734000 & -1.243076000 & 3.699822000 \\ 6 & 1.674626000 & -0.897778000 & 2.873209000 \\ 6 & 2.498607000 & -0.761363000 & 1.801122000 \\ 8 & 6.306991000 & 1.970205000 & -0.451702000 \\ 6 & 6.423266000 & 3.047715000 & -1.231923000 \\ 6 & 5.434379000 & 4.142364000 & -0.941247000 \\ 8 & 4.120078000 & 3.650630000 & -1.094781000 \\ 6 & 5.591430000 & 4.705489000 & 0.471401000 \\ 8 & 6.409208000 & 5.845648000 & 0.486512000 \\ 6 & 4.181455000 & 5.059353000 & 0.834069000 \\ 6 & 3.368093000 & 4.073270000 & 0.020788000 \\ 1 & 5.892066000 & -3.794134000 & 1.804669000 \\ 1 & 5.141939000 & -4.267916000 & -0.310457000 \\ 1 & 6.589848000 & -3.195668000 & -0.268989000 \\ 1 & 4.811551000 & -2.430937000 & -1.871059000 \\ 1 & 5.498061000 & -0.712894000 & 0.509029000 \\ 1 & 4.021476000 & 0.534119000 & -1.702806000 \\ 1 & 3.977443000 & 0.843968000 & 0.070363000 \\ 1 & 2.313101000 & -0.909790000 & -1.566846000 \\ 1 & -0.282628000 & -1.032080000 & 4.605808000 \\ 1 & -1.614585000 & -1.106054000 & 3.551299000 \\ 1 & 2.085608000 & -0.951012000 & 3.887548000 \\ 1 & 3.586946000 & -0.711592000 & 1.955552000 \\ 1 & 7.427460000 & 3.460175000 & -1.206944000 \\ 1 & 6.360383000 & 2.797566000 & -2.287038000 \\ 1 & 5.580518000 & 4.961219000 & -1.682583000 \\ 1 & 5.968462000 & 3.939108000 & 1.190062000 \\ 1 & 7.343294000 & 5.520224000 & 0.404937000 \\ 1 & 3.940473000 & 6.097658000 & 0.512520000 \\ 1 & 3.993964000 & 4.949077000 & 1.924525000 \\ 1 & 3.119500000 & 3.185882000 & 0.642521000 \\ 1 & 2.420339000 & 4.540711000 & -0.321435000 \\ 11 & 10.186749000 & -1.137371000 & -0.363752000 \\ 8 & 10.507615000 & 0.422366000 & 1.440224000 \\ 8 & 8.678113000 & -2.234095000 & 1.249972000 \\ 8 & 8.627268000 & -1.840596000 & -2.221369000 \\ 1 & 9.557999000 & 0.643123000 & 1.323661000 \\ 1 & 10.943158000 & 0.968150000 & 0.759650000\end{array}$




$\begin{array}{lccc}1 & 9.174244000 & -2.057928000 & 2.065053000 \\ 1 & 8.191667000 & -1.388142000 & 1.069838000 \\ 1 & 7.686728000 & -1.766778000 & -1.967505000 \\ 1 & 8.757389000 & -0.975732000 & -2.656609000 \\ 8 & 10.849929000 & 0.914482000 & -1.334604000 \\ 1 & 9.928695000 & 1.133410000 & -1.635469000 \\ 1 & 11.425184000 & 1.023968000 & -2.105510000 \\ 7 & -7.036340000 & 0.052552000 & 0.467502000 \\ 6 & -7.434413000 & 0.170925000 & 1.830107000 \\ 7 & -6.384022000 & -0.048508000 & 2.625300000 \\ 6 & -5.271605000 & -0.323186000 & 1.814708000 \\ 6 & -3.898602000 & -0.617914000 & 2.147163000 \\ 8 & -3.348570000 & -0.689804000 & 3.245029000 \\ 7 & -3.061600000 & -0.859935000 & 1.003335000 \\ 6 & -3.557527000 & -0.781288000 & -0.314779000 \\ 7 & -2.709453000 & -1.147676000 & -1.365328000 \\ 7 & -4.842606000 & -0.478580000 & -0.626715000 \\ 6 & -5.667652000 & -0.261403000 & 0.467788000 \\ 6 & -10.368609000 & 1.889881000 & 0.891045000 \\ 6 & -9.944970000 & 1.315858000 & -0.478820000 \\ 8 & -8.535703000 & 1.462008000 & -0.660814000 \\ 6 & -10.309403000 & -0.195416000 & -0.593877000 \\ 6 & -8.962400000 & -0.898381000 & -0.862509000 \\ 6 & -7.891107000 & 0.198557000 & -0.733278000 \\ 8 & -10.250258000 & 3.281318000 & 0.962753000 \\ 6 & -13.924966000 & 0.781476000 & 0.048048000 \\ 8 & -12.824941000 & -2.135610000 & -0.382249000 \\ 15 & -12.733987000 & -1.109382000 & -1.397068000 \\ 8 & -11.200982000 & -0.400448000 & -1.654198000 \\ 8 & -13.094808000 & -1.515040000 & -2.961114000 \\ 8 & -13.706407000 & 0.269148000 & -1.224736000 \\ 1 & -8.446821000 & 0.434444000 & 2.162513000 \\ 1 & -2.047188000 & -0.987099000 & 1.146253000 \\ 1 & -3.010492000 & -0.854393000 & -2.265627000 \\ 1 & -1.718426000 & -1.032123000 & -1.202868000 \\ 1 & -11.453167000 & 1.718986000 & 1.056226000 \\ 1 & -9.809500000 & 1.402124000 & 1.721499000 \\ 1 & -10.377361000 & 1.926984000 & -1.304412000 \\ 1 & -10.770938000 & -0.568414000 & 0.352691000 \\ 1 & -8.926758000 & -1.344531000 & -1.875437000 \\ 1 & -8.799109000 & -1.739831000 & -0.163250000 \\ 1 & -7.224180000 & 0.265383000 & -1.625376000 \\ 1 & -9.334551000 & 3.496440000 & 0.832578000 \\ 1 & -14.599378000 & 1.631562000 & -0.092620000 \\ 1 & -12.984450000 & 1.136182000 & 0.504351000 \\ 1 & -14.397245000 & 0.043169000 & 0.706882000\end{array}$




$\begin{array}{cccc}1 & -12.957415000 & -0.844345000 & -3.612283000 \\ 8 & 9.569439000 & -4.116285000 & -0.731145000 \\ 1 & 9.236677000 & -3.622497000 & -1.504824000 \\ 1 & 9.127486000 & -3.673649000 & 0.022926000 \\ 8 & 11.853517000 & -2.683264000 & -0.637236000 \\ 1 & 11.189706000 & -3.420758000 & -0.670934000 \\ 1 & 12.481184000 & -2.919626000 & 0.060790000\end{array}$

\begin{tabular}{|c|c|c|c|}
\hline \multicolumn{4}{|c|}{$\left(\mathrm{H}_{2} \mathrm{O}\right)_{2}{ }^{\bullet \bullet}{ }^{-} \mathrm{PSG} \bullet \bullet \mathrm{CSP}^{-} \bullet \bullet \mathrm{Na}\left(\mathrm{H}_{2} \mathrm{O}\right)_{5}$} \\
\hline 8 & 5.668306000 & -2.874200000 & 2.241153000 \\
\hline 6 & 5.852841000 & -3.384891000 & 0.948864000 \\
\hline 6 & 5.150850000 & -2.496498000 & -0.086365000 \\
\hline 8 & 3.790633000 & -2.348258000 & 0.242906000 \\
\hline 6 & 5.765210000 & -1.132541000 & -0.192652000 \\
\hline 8 & 6.816508000 & -1.091900000 & 4000 \\
\hline 6 & 4.630715000 & -0.278711000 & 1000 \\
\hline 6 & 3.385808000 & 3606000 & 50000 \\
\hline 15 & 8.150308000 & -0.281261000 & -0.506658000 \\
\hline 8 & 8.558635000 & -0.716802000 & 0.887767000 \\
\hline 8 & 9.163783000 & -0.459482000 & 1000 \\
\hline 7 & 2.526309000 & -0.417009000 & 7000 \\
\hline 6 & 1.088517000 & 20000 & 000 \\
\hline 8 & 0.576473000 & & 8000 \\
\hline 7 & 0.2451700 & -0.439700000 & 000 \\
\hline 6 & 0.783318000 & -0.177226000 & 000 \\
\hline 7 & -0.097273000 & -0.1 & 000 \\
\hline 6 & 2.213198000 & -0.070322000 & 9000 \\
\hline 6 & 3.039893000 & -0.250462000 & 8000 \\
\hline 8 & 7.680556000 & 921000 & 0000 \\
\hline 6 & 7.420093000 & 382000 & 3000 \\
\hline 6 & 6.863370000 & 3.320893000 & 77000 \\
\hline 8 & 5.65497400 & 3.257988000 & 52000 \\
\hline 6 & 7.83820200 & 4.246743000 & -0.5 \\
\hline 8 & 8.592680000 & 5.016465000 & 3000 \\
\hline 6 & 6.917684000 & 622000 & 1000 \\
\hline 6 & 5.723286000 & 4.214432000 & 4000 \\
\hline 1 & 6.161570000 & -3.482718000 & 2.850217000 \\
\hline 1 & 5.424734000 & -4.410448000 & 2015000 \\
\hline 1 & 6.939412000 & -3.444571000 & 0.713559000 \\
\hline 1 & 5.216149000 & -3.006987000 & -1.075519000 \\
\hline 1 & 6.000066000 & -0.796532000 & 0.815919000 \\
\hline 1 & 4.675161000 & -0.099672000 & -1.724876000 \\
\hline 1 & 4.645727000 & 0.711138000 & -0.117970000 \\
\hline 1 & 2.846945000 & -1.296393000 & -1.238366000 \\
\hline 1 & 0.251210000 & 0.321312000 & 4.714482000 \\
\hline & -1.085432000 & 0.008798000 & 3.708647000 \\
\hline
\end{tabular}




$\begin{array}{llcc}1 & 2.621112000 & 0.130028000 & 4.023312000 \\ 1 & 4.127799000 & -0.201171000 & 2.115811000 \\ 1 & 8.294773000 & 1.995013000 & -2.123044000 \\ 1 & 6.762024000 & 1.379008000 & -2.143036000 \\ 1 & 6.633636000 & 3.746440000 & -2.305685000 \\ 1 & 8.506536000 & 3.688400000 & 0.126263000 \\ 1 & 9.285638000 & 4.411137000 & -1.842838000 \\ 1 & 6.604975000 & 6.007567000 & -0.361608000 \\ 1 & 7.380765000 & 5.440720000 & 1.185789000 \\ 1 & 5.830892000 & 3.684245000 & 1.429384000 \\ 1 & 4.785621000 & 4.809442000 & 0.481027000 \\ 11 & 11.321305000 & -1.316464000 & -0.506420000 \\ 8 & 9.682082000 & -3.186975000 & -2.283157000 \\ 1 & 9.578989000 & -3.449725000 & -1.346062000 \\ 1 & 9.308764000 & -2.278896000 & -2.292527000 \\ 8 & 11.202132000 & 0.112896000 & 1.336198000 \\ 1 & 10.226510000 & 0.007776000 & 1.409636000 \\ 1 & 11.320449000 & 0.886972000 & 0.757187000 \\ 8 & 12.306892000 & -2.657154000 & -2.065194000 \\ 1 & 11.402437000 & -2.984489000 & -2.327358000 \\ 1 & 12.729992000 & -2.371239000 & -2.887148000 \\ 8 & 9.960123000 & -2.976727000 & 0.531471000 \\ 1 & 10.187094000 & -3.493938000 & 1.318009000 \\ 1 & 9.336636000 & -2.260122000 & 0.830502000 \\ 8 & 11.435003000 & 0.949276000 & -1.391736000 \\ 1 & 10.532593000 & 0.654059000 & -1.669656000 \\ 1 & 11.850750000 & 1.335302000 & -2.175894000 \\ 7 & -6.493838000 & 0.436816000 & 0.448813000 \\ 6 & -6.865267000 & 0.958804000 & 1.715151000 \\ 7 & -5.812015000 & 0.942213000 & 2.541691000 \\ 6 & -4.721084000 & 0.411114000 & 1.837785000 \\ 6 & -3.355098000 & 0.181790000 & 2.235274000 \\ 8 & -2.791357000 & 0.406436000 & 3.308903000 \\ 7 & -2.537717000 & -0.395181000 & 1.207388000 \\ 6 & -3.045094000 & -0.672915000 & -0.082820000 \\ 7 & -2.201270000 & -1.341032000 & -0.992239000 \\ 7 & -4.317335000 & -0.438488000 & -0.460866000 \\ 6 & -5.136621000 & 0.099475000 & 0.529454000 \\ 6 & -9.487296000 & 2.750271000 & 0.253759000 \\ 6 & -9.216622000 & 1.753786000 & -0.891315000 \\ 8 & -7.806277000 & 1.608299000 & -1.091530000 \\ 6 & -9.832970000 & 0.350586000 & -0.571892000 \\ 6 & -8.598913000 & -0.589351000 & -0.512785000 \\ 6 & -7.373049000 & 0.304603000 & -0.746794000 \\ 8 & -9.065453000 & 4.058486000 & -0.020916000 \\ 6 & -13.165420000 & 1.879525000 & -0.126398000\end{array}$




$\begin{array}{lccc}8 & -12.453783000 & -1.019745000 & 0.202799000 \\ 15 & -12.383627000 & -0.479780000 & -1.198674000 \\ 8 & -10.714021000 & -0.034931000 & -1.562154000 \\ 8 & -12.890028000 & -1.235898000 & -2.391039000 \\ 8 & -13.096959000 & 1.109694000 & -1.265838000 \\ 1 & -7.868627000 & 1.340942000 & 1.954583000 \\ 1 & -1.528969000 & -0.506387000 & 1.374461000 \\ 1 & -2.504584000 & -1.289163000 & -1.937709000 \\ 1 & -1.213026000 & -1.162091000 & -0.878498000 \\ 1 & -10.581167000 & 2.856646000 & 0.412525000 \\ 1 & -9.032419000 & 2.398359000 & 1.208081000 \\ 1 & -9.572828000 & 2.167386000 & -1.861721000 \\ 1 & -10.349467000 & 0.370528000 & 0.419344000 \\ 1 & -8.651874000 & -1.372378000 & -1.293318000 \\ 1 & -8.556878000 & -1.131653000 & 0.449906000 \\ 1 & -6.738700000 & -0.028969000 & -1.600744000 \\ 1 & -8.149560000 & 4.012818000 & -0.266735000 \\ 1 & -13.784434000 & 2.750478000 & -0.373190000 \\ 1 & -12.168744000 & 2.242132000 & 0.185025000 \\ 1 & -13.623339000 & 1.347197000 & 0.717616000 \\ 8 & -13.760280000 & -3.385377000 & 0.691685000 \\ 1 & -13.809309000 & -3.683447000 & -0.223074000 \\ 1 & -13.277776000 & -2.544739000 & 0.690120000 \\ 8 & -14.022103000 & -3.666285000 & -2.044369000 \\ 1 & -14.874216000 & -3.335201000 & -1.779937000 \\ 1 & -13.477831000 & -2.895489000 & -2.285618000\end{array}$

$\begin{array}{lccc}\left.\mathbf{( H}_{\mathbf{2}} \mathbf{O}\right)_{\mathbf{2}^{\bullet}{ }^{-} \mathbf{P S G} \boldsymbol{\bullet} \bullet \mathbf{C S P}^{-}} \boldsymbol{\bullet \bullet}^{-} \mathbf{N a}\left(\mathbf{H}_{\mathbf{2}} \mathbf{O}\right)_{\mathbf{6}} & \\ 8 & 5.809325000 & -2.988752000 & 1.591177000 \\ 6 & 5.962712000 & -3.272624000 & 0.227116000 \\ 6 & 5.206121000 & -2.246581000 & -0.625772000 \\ 8 & 3.847950000 & -2.222534000 & -0.257234000 \\ 6 & 5.760596000 & -0.858087000 & -0.501100000 \\ 8 & 6.780949000 & -0.614192000 & -1.336823000 \\ 6 & 4.575676000 & 0.014326000 & -0.718625000 \\ 6 & 3.378020000 & -0.920328000 & -0.527704000 \\ 15 & 7.879471000 & 0.559401000 & -0.879189000 \\ 8 & 8.479633000 & 0.167555000 & 0.461082000 \\ 8 & 8.794136000 & 0.697132000 & -2.076204000 \\ 7 & 2.510993000 & -0.471181000 & 0.543607000 \\ 6 & 1.078889000 & -0.679624000 & 0.372029000 \\ 8 & 0.555235000 & -0.690839000 & -0.738007000 \\ 7 & 0.251560000 & -0.778042000 & 1.512860000 \\ 6 & 0.803942000 & -0.747765000 & 2.743109000 \\ 7 & -0.056197000 & -0.944286000 & 3.801431000 \\ 6 & 2.231356000 & -0.618528000 & 2.933237000\end{array}$




$\begin{array}{lccc}6 & 3.043737000 & -0.545454000 & 1.847890000 \\ 8 & 6.939743000 & 1.871447000 & -0.614057000 \\ 6 & 7.068558000 & 2.883776000 & -1.475620000 \\ 6 & 6.127283000 & 4.032255000 & -1.240317000 \\ 8 & 4.792864000 & 3.578886000 & -1.320356000 \\ 6 & 6.346379000 & 4.692018000 & 0.121298000 \\ 8 & 7.203306000 & 5.799327000 & 0.028416000 \\ 6 & 4.961109000 & 5.123491000 & 0.495021000 \\ 6 & 4.089525000 & 4.111479000 & -0.220295000 \\ 1 & 6.347707000 & -3.667241000 & 2.075457000 \\ 1 & 5.557780000 & -4.286751000 & 0.013212000 \\ 1 & 7.040204000 & -3.263611000 & -0.046711000 \\ 1 & 5.271191000 & -2.572163000 & -1.689942000 \\ 1 & 6.037476000 & -0.690821000 & 0.537654000 \\ 1 & 4.577953000 & 0.423908000 & -1.754222000 \\ 1 & 4.560526000 & 0.873462000 & -0.011345000 \\ 1 & 2.827577000 & -0.951058000 & -1.494761000 \\ 1 & 0.291242000 & -0.669394000 & 4.687725000 \\ 1 & -1.051665000 & -0.826135000 & 3.655774000 \\ 1 & 2.650426000 & -0.604600000 & 3.945226000 \\ 1 & 4.132466000 & -0.480000000 & 1.989906000 \\ 1 & 8.086504000 & 3.260475000 & -1.509013000 \\ 1 & 6.965283000 & 2.559384000 & -2.507081000 \\ 1 & 6.278957000 & 4.788294000 & -2.044604000 \\ 1 & 6.718078000 & 3.967434000 & 0.884730000 \\ 1 & 8.122766000 & 5.434607000 & -0.053142000 \\ 1 & 4.747036000 & 6.143761000 & 0.104182000 \\ 1 & 4.802682000 & 5.100242000 & 1.595362000 \\ 1 & 3.827276000 & 3.282688000 & 0.472672000 \\ 1 & 3.149747000 & 4.588056000 & -0.571839000 \\ 11 & 10.751835000 & -1.340114000 & -0.333525000 \\ 8 & 11.129490000 & 0.401484000 & 1.267758000 \\ 8 & 9.200848000 & -2.276795000 & 1.323750000 \\ 8 & 9.127828000 & -2.117517000 & -2.131210000 \\ 1 & 10.175081000 & 0.608054000 & 1.161596000 \\ 1 & 11.538672000 & 0.867336000 & 0.514967000 \\ 1 & 9.641375000 & -2.087725000 & 2.166876000 \\ 1 & 8.739504000 & -1.429554000 & 1.086206000 \\ 1 & 8.189911000 & -2.031026000 & -1.874003000 \\ 1 & 9.256369000 & -1.269148000 & -2.600393000 \\ 8 & 11.410069000 & 0.583013000 & -1.547519000 \\ 1 & 10.484849000 & 0.807234000 & -1.835610000 \\ 7 & 11.961965000 & 0.610835000 & -2.342368000 \\ 7 & -6.514590000 & 0.202428000 & 0.627143000 \\ & -6.878194000 & 0.414256000 & 1.982518000 \\ & -5.815363000 & 0.224934000 & 2.774532000\end{array}$




$\begin{array}{lccc}6 & -4.725659000 & -0.113567000 & 1.958613000 \\ 6 & -3.351377000 & -0.403110000 & 2.281433000 \\ 8 & -2.777845000 & -0.415150000 & 3.373066000 \\ 7 & -2.538648000 & -0.719969000 & 1.142548000 \\ 6 & -3.058385000 & -0.705555000 & -0.172471000 \\ 7 & -2.218797000 & -1.138626000 & -1.217391000 \\ 7 & -4.338037000 & -0.410263000 & -0.476364000 \\ 6 & -5.152291000 & -0.124132000 & 0.617287000 \\ 6 & -9.536004000 & 2.457366000 & 0.996005000 \\ 6 & -9.259487000 & 1.769831000 & -0.356166000 \\ 8 & -7.848877000 & 1.689298000 & -0.587350000 \\ 6 & -9.863003000 & 0.325647000 & -0.385518000 \\ 6 & -8.619150000 & -0.590823000 & -0.535923000 \\ 6 & -7.402530000 & 0.345187000 & -0.560679000 \\ 8 & -9.125326000 & 3.796631000 & 1.046054000 \\ 6 & -13.201804000 & 1.659086000 & 0.412001000 \\ 8 & -12.475785000 & -1.225498000 & -0.003282000 \\ 15 & -12.400162000 & -0.346454000 & -1.220678000 \\ 8 & -10.730160000 & 0.178358000 & -1.449550000 \\ 8 & -12.897605000 & -0.775673000 & -2.569431000 \\ 8 & -13.118429000 & 1.206709000 & -0.885729000 \\ 1 & -7.883496000 & 0.715631000 & 2.311743000 \\ 1 & -1.525371000 & -0.843417000 & 1.270229000 \\ 1 & -2.534885000 & -0.879703000 & -2.123846000 \\ 1 & -1.231250000 & -0.976579000 & -1.076088000 \\ 1 & -10.630409000 & 2.512973000 & 1.175690000 \\ 1 & -9.076495000 & 1.889229000 & 1.837114000 \\ 1 & -9.621520000 & 2.402923000 & -1.197446000 \\ 1 & -10.389650000 & 0.105187000 & 0.575555000 \\ 1 & -8.663915000 & -1.180272000 & -1.471663000 \\ 1 & -8.571474000 & -1.333917000 & 0.281555000 \\ 1 & -6.772452000 & 0.227459000 & -1.472869000 \\ 1 & -8.209744000 & 3.819400000 & 0.795879000 \\ 1 & -13.835726000 & 2.553745000 & 0.392219000 \\ 1 & -12.211681000 & 1.947196000 & 0.810164000 \\ 1 & -13.650683000 & 0.921770000 & 1.090419000 \\ 8 & -13.741730000 & -3.658107000 & -0.157144000 \\ 1 & -13.784576000 & -3.710971000 & -1.118132000 \\ 1 & -13.273341000 & -2.837824000 & 0.060385000 \\ 8 & -13.977123000 & -3.232336000 & -2.878854000 \\ 1 & -14.832974000 & -2.993323000 & -2.538095000 \\ 1 & -13.443525000 & -2.418177000 & -2.908875000 \\ 8 & 10.038604000 & -4.311273000 & -0.517605000 \\ 1 & 9.721173000 & -3.858679000 & -1.322506000 \\ & 12.371301000 & -2.957591000 & -0.483514000\end{array}$




$\begin{array}{rrrr}1 & 11.676599000 & -3.667264000 & -0.480538000 \\ 1 & 12.964394000 & -3.162342000 & 0.253768000\end{array}$

\section{$\mathrm{HPS}^{\circ} \bullet \mathrm{CSP}^{-} \bullet \bullet \mathbf{K}\left(\mathrm{H}_{2} \mathrm{O}\right)_{5}$}

$\begin{array}{lccc}8 & 5.659595000 & 3.621574000 & -1.500241000 \\ 6 & 6.358390000 & 3.175355000 & -0.370712000 \\ 6 & 5.417461000 & 2.429837000 & 0.584795000 \\ 8 & 4.412542000 & 3.312456000 & 1.041264000 \\ 6 & 4.768384000 & 1.200506000 & -0.077287000 \\ 8 & 4.830791000 & 0.099674000 & 0.785699000 \\ 6 & 3.331878000 & 1.618589000 & -0.227516000 \\ 6 & 3.170652000 & 2.671017000 & 0.857739000 \\ 15 & 6.135798000 & -0.872525000 & 0.498620000 \\ 8 & 7.375094000 & -0.056879000 & 0.148093000 \\ 8 & 6.221186000 & -1.828477000 & 1.659755000 \\ 7 & 2.136706000 & 3.629927000 & 0.529796000 \\ 6 & 0.759077000 & 3.180007000 & 0.667366000 \\ 8 & 0.446760000 & 2.282084000 & 1.441530000 \\ 7 & -0.257566000 & 3.833309000 & -0.067566000 \\ 6 & 0.071116000 & 4.818435000 & -0.927592000 \\ 7 & -0.980829000 & 5.477114000 & -1.530114000 \\ 6 & 1.444457000 & 5.210869000 & -1.147761000 \\ 6 & 2.435162000 & 4.584401000 & -0.459781000 \\ 8 & 5.774926000 & -1.649324000 & -0.926006000 \\ 6 & 4.674843000 & -2.497410000 & -0.786460000 \\ 6 & 4.364594000 & -3.166825000 & -2.129634000 \\ 8 & 4.196221000 & -2.183138000 & -3.129177000 \\ 6 & 5.461390000 & -4.145126000 & -2.559830000 \\ 8 & 5.114861000 & -5.472604000 & -2.264872000 \\ 6 & 5.529156000 & -3.933751000 & -4.042022000 \\ 6 & 5.056296000 & -2.504950000 & -4.197518000 \\ 19 & 8.883279000 & -2.322087000 & 2.003628000 \\ 8 & 8.577949000 & -1.347800000 & -1.962087000 \\ 8 & 8.615182000 & -2.794445000 & 4.610193000 \\ 8 & 8.844059000 & 0.432201000 & 2.327776000 \\ 1 & 6.323640000 & 4.095188000 & -2.065351000 \\ 1 & 6.801142000 & 4.045869000 & 0.163180000 \\ 1 & 7.185776000 & 2.499994000 & -0.682551000 \\ 1 & 6.021325000 & 2.111236000 & 1.463868000 \\ 1 & 5.179401000 & 0.977420000 & -1.092435000 \\ 1 & 2.624326000 & 0.771384000 & -0.088313000 \\ 1 & 3.175753000 & 2.061434000 & -1.236409000 \\ 1 & 2.919605000 & 2.164612000 & 1.818124000 \\ 1 & -0.747368000 & 5.992025000 & -2.344170000 \\ 1 & -1.893025000 & 5.039084000 & -1.530849000 \\ 1 & 1.679024000 & 5.997926000 & -1.872471000\end{array}$




$\begin{array}{lccc}1 & 3.480034000 & 4.874041000 & -0.635682000 \\ 1 & 4.875403000 & -3.289600000 & -0.029932000 \\ 1 & 3.774246000 & -1.920388000 & -0.476561000 \\ 1 & 3.401506000 & -3.716363000 & -2.029830000 \\ 1 & 6.450024000 & -3.890962000 & -2.110174000 \\ 1 & 5.239925000 & -5.582936000 & -1.286396000 \\ 1 & 4.828133000 & -4.618000000 & -4.570969000 \\ 1 & 6.560386000 & -4.071539000 & -4.434272000 \\ 1 & 5.926601000 & -1.812970000 & -4.179556000 \\ 1 & 4.521028000 & -2.376201000 & -5.162183000 \\ 1 & 8.257740000 & -0.705245000 & -1.284851000 \\ 1 & 7.749710000 & -1.797300000 & -2.196223000 \\ 1 & 7.980439000 & -2.029953000 & 4.645269000 \\ 1 & 8.212153000 & -3.482371000 & 5.158025000 \\ 1 & 9.340898000 & 1.262194000 & 2.360433000 \\ 1 & 8.313362000 & 0.451917000 & 1.485482000 \\ 8 & 6.958412000 & -0.697036000 & 4.143057000 \\ 1 & 7.565945000 & -0.070790000 & 3.697011000 \\ 1 & 6.430716000 & -1.057891000 & 3.396919000 \\ 7 & -6.388091000 & 0.713286000 & -0.363546000 \\ 6 & -6.961882000 & 1.468684000 & -1.426301000 \\ 7 & -6.124545000 & 2.444807000 & -1.786567000 \\ 6 & -4.983593000 & 2.364608000 & -0.972707000 \\ 6 & -3.776491000 & 3.153861000 & -0.942071000 \\ 8 & -3.434444000 & 4.103155000 & -1.646607000 \\ 7 & -2.842233000 & 2.751022000 & 0.073236000 \\ 6 & -3.094285000 & 1.657229000 & 0.929457000 \\ 7 & -2.193491000 & 1.405343000 & 1.968248000 \\ 7 & -4.218130000 & 0.898859000 & 0.880183000 \\ 6 & -5.137936000 & 1.287708000 & -0.082781000 \\ 6 & -12.160883000 & -3.585230000 & -0.321061000 \\ 6 & -9.238126000 & -1.139921000 & -2.100732000 \\ 6 & -8.708579000 & -1.638541000 & -0.737529000 \\ 8 & -7.299373000 & -1.429256000 & -0.647933000 \\ 6 & -9.398944000 & -0.894595000 & 0.445031000 \\ 8 & -9.951619000 & -1.818742000 & 1.341261000 \\ 6 & -8.258987000 & -0.105302000 & 1.122911000 \\ 6 & -6.984136000 & -0.450048000 & 0.332136000 \\ 15 & -11.629269000 & -1.894540000 & 1.653627000 \\ 8 & -11.594348000 & -2.238110000 & 3.273420000 \\ 8 & -12.435246000 & -0.776235000 & 1.213066000 \\ 8 & -11.990550000 & -3.434909000 & 1.050165000 \\ 1 & -8.807985000 & -1.928553000 & -3.173070000 \\ 1 & -7.933245000 & 1.252679000 & -1.889703000 \\ 1 & -1.914707000 & 3.200991000 & 0.101096000 \\ & -2.279308000 & 0.509626000 & 2.389599000\end{array}$




$\begin{array}{cccc}1 & -1.239649000 & 1.704591000 & 1.820909000 \\ 1 & -12.290079000 & -4.659869000 & -0.481783000 \\ 1 & -11.280254000 & -3.236138000 & -0.878503000 \\ 1 & -13.053348000 & -3.053151000 & -0.671710000 \\ 1 & -10.337775000 & -1.235112000 & -2.145742000 \\ 1 & -8.975919000 & -0.070556000 & -2.267180000 \\ 1 & -8.808690000 & -2.746967000 & -0.668083000 \\ 1 & -10.198136000 & -0.207236000 & 0.076733000 \\ 1 & -8.134808000 & -0.387626000 & 2.186216000 \\ 1 & -8.475447000 & 0.979802000 & 1.128027000 \\ 1 & -6.192117000 & -0.918234000 & 0.963910000 \\ 1 & -11.041317000 & -2.956177000 & 3.540256000 \\ 1 & -7.859633000 & -1.885009000 & -3.196871000 \\ 8 & 10.018682000 & -2.919540000 & -0.297738000 \\ 1 & 10.912545000 & -3.023516000 & -0.651919000 \\ 1 & 9.525423000 & -2.405175000 & -0.993914000\end{array}$

\section{HPS $\bullet \mathrm{CSP}^{-} \cdot \bullet \mathbf{K}\left(\mathrm{H}_{\mathbf{2}} \mathrm{O}\right)_{\mathbf{6}}$}

$\begin{array}{lccc}8 & -5.460459000 & 3.613965000 & 1.272363000 \\ 6 & -6.111552000 & 3.193143000 & 0.105181000 \\ 6 & -5.134615000 & 2.455349000 & -0.819948000 \\ 8 & -4.104242000 & 3.337407000 & -1.216131000 \\ 6 & -4.524615000 & 1.210159000 & -0.150200000 \\ 8 & -4.576336000 & 0.116196000 & -1.023672000 \\ 6 & -3.087363000 & 1.604017000 & 0.051996000 \\ 6 & -2.877155000 & 2.676688000 & -1.004565000 \\ 15 & -5.893908000 & -0.846995000 & -0.774833000 \\ 8 & -7.145510000 & -0.010356000 & -0.511799000 \\ 8 & -5.913900000 & -1.851413000 & -1.894771000 \\ 7 & -1.844438000 & 3.616317000 & -0.621813000 \\ 6 & -0.467370000 & 3.158196000 & -0.733534000 \\ 8 & -0.142456000 & 2.275487000 & -1.520357000 \\ 7 & 0.534227000 & 3.787093000 & 0.041410000 \\ 6 & 0.189704000 & 4.753812000 & 0.916747000 \\ 7 & 1.229539000 & 5.391441000 & 1.559781000 \\ 6 & -1.186704000 & 5.148909000 & 1.113139000 \\ 6 & -2.163232000 & 4.546911000 & 0.384693000 \\ 8 & -5.619764000 & -1.555595000 & 0.701641000 \\ 6 & -4.580668000 & -2.485328000 & 0.647506000 \\ 6 & -4.311729000 & -3.041308000 & 2.049305000 \\ 8 & -4.020554000 & -1.983939000 & 2.938064000 \\ 6 & -5.493634000 & -3.843170000 & 2.595367000 \\ 8 & -5.340255000 & -5.217647000 & 2.357744000 \\ 6 & -5.432251000 & -3.549187000 & 4.063269000 \\ 6 & -4.784124000 & -2.180488000 & 4.106769000 \\ 19 & -9.457471000 & -1.634367000 & -1.088766000\end{array}$




$\begin{array}{cccc}8 & -7.901693000 & -0.301044000 & 2.135551000 \\ 8 & -9.427309000 & -3.071383000 & -3.451765000 \\ 8 & -8.742773000 & 0.706124000 & -2.508254000 \\ 1 & -6.146863000 & 4.078011000 & 1.818393000 \\ 1 & -6.522475000 & 4.076587000 & -0.432671000 \\ 1 & -6.957958000 & 2.520753000 & 0.367840000 \\ 1 & -5.700647000 & 2.155986000 & -1.730299000 \\ 1 & -4.971333000 & 0.987710000 & 0.849812000 \\ 1 & -2.387810000 & 0.749692000 & -0.083951000 \\ 1 & -2.955665000 & 2.022359000 & 1.074758000 \\ 1 & -2.600108000 & 2.187287000 & -1.966601000 \\ 1 & 0.980168000 & 5.891226000 & 2.378433000 \\ 1 & 2.140858000 & 4.951792000 & 1.569084000 \\ 1 & -1.435082000 & 5.917537000 & 1.852981000 \\ 1 & -3.211019000 & 4.836598000 & 0.542766000 \\ 1 & -4.841882000 & -3.330444000 & -0.028195000 \\ 1 & -3.643441000 & -2.006216000 & 0.282935000 \\ 1 & -3.414383000 & -3.698654000 & 1.997126000 \\ 1 & -6.468856000 & -3.480577000 & 2.192281000 \\ 1 & -5.560193000 & -5.362014000 & 1.400706000 \\ 1 & -4.782610000 & -4.286734000 & 4.586240000 \\ 1 & -6.443044000 & -3.541816000 & 4.526519000 \\ 1 & -5.566708000 & -1.392855000 & 4.159653000 \\ 1 & -4.130958000 & -2.088886000 & 5.000571000 \\ 1 & -7.661010000 & 0.048521000 & 1.247426000 \\ 1 & -7.260814000 & -1.026431000 & 2.221309000 \\ 1 & -8.733703000 & -2.460948000 & -3.842644000 \\ 1 & -9.866054000 & -3.496038000 & -4.202637000 \\ 1 & -8.912160000 & 1.641357000 & -2.692731000 \\ 1 & -8.051143000 & 0.676065000 & -1.796030000 \\ 8 & -7.556231000 & -1.300320000 & -4.102806000 \\ 1 & -7.936406000 & -0.459226000 & -3.770705000 \\ 1 & -6.831629000 & -1.497397000 & -3.465347000 \\ 7 & 6.657839000 & 0.655613000 & 0.383366000 \\ 6 & 7.211223000 & 1.384853000 & 1.474734000 \\ 7 & 6.366528000 & 2.351512000 & 1.843333000 \\ 6 & 5.241446000 & 2.291568000 & 1.006066000 \\ 6 & 4.035140000 & 3.081887000 & 0.971665000 \\ 8 & 3.679939000 & 4.014291000 & 1.691994000 \\ 7 & 3.120409000 & 2.704165000 & -0.070769000 \\ 6 & 3.388913000 & 1.631512000 & -0.948352000 \\ 7 & 2.507544000 & 1.405170000 & -2.010082000 \\ 6 & 4.511630000 & 0.872158000 & -0.896624000 \\ 6 & 5.413176000 & 1.236996000 & 0.092944000 \\ & 12.456670000 & -3.608078000 & 0.332363000 \\ & 9.483016000 & -1.235715000 & 2.121547000\end{array}$




$\begin{array}{lccc}6 & 8.980337000 & -1.697070000 & 0.735250000 \\ 8 & 7.571929000 & -1.490859000 & 0.626462000 \\ 6 & 9.688381000 & -0.916911000 & -0.413071000 \\ 8 & 10.265672000 & -1.812768000 & -1.322357000 \\ 6 & 8.556262000 & -0.117637000 & -1.092419000 \\ 6 & 7.269645000 & -0.487219000 & -0.332572000 \\ 15 & 11.949633000 & -1.866895000 & -1.604464000 \\ 8 & 11.948188000 & -2.165418000 & -3.233572000 \\ 8 & 12.737596000 & -0.754931000 & -1.117851000 \\ 8 & 12.311656000 & -3.420716000 & -1.037248000 \\ 8 & 9.037343000 & -2.056644000 & 3.162818000 \\ 1 & 8.173687000 & 1.157500000 & 1.951167000 \\ 1 & 2.194136000 & 3.156069000 & -0.106459000 \\ 1 & 2.600102000 & 0.518647000 & -2.449133000 \\ 1 & 1.551272000 & 1.701413000 & -1.872635000 \\ 1 & 12.595105000 & -4.685369000 & 0.465190000 \\ 1 & 11.561123000 & -3.284740000 & 0.881496000 \\ 1 & 13.335755000 & -3.076368000 & 0.715836000 \\ 1 & 10.582106000 & -1.327759000 & 2.183665000 \\ 1 & 9.213126000 & -0.172610000 & 2.313796000 \\ 1 & 9.086807000 & -2.802639000 & 0.635904000 \\ 1 & 10.474803000 & -0.233995000 & -0.010555000 \\ 1 & 8.452302000 & -0.375841000 & -2.164001000 \\ 1 & 8.766823000 & 0.968368000 & -1.068690000 \\ 1 & 6.489913000 & -0.941016000 & -0.989576000 \\ 1 & 11.408514000 & -2.881863000 & -3.530402000 \\ 1 & 8.088561000 & -2.017101000 & 3.171312000 \\ 8 & -10.301781000 & -1.352769000 & 1.396906000 \\ 1 & -10.670369000 & -1.946041000 & 2.065890000 \\ 1 & -9.490578000 & -0.958292000 & 1.813193000 \\ 8 & -7.860240000 & -3.851221000 & -1.258887000 \\ 1 & -8.300359000 & -3.959051000 & -2.125899000 \\ 1 & -7.030713000 & -3.378438000 & -1.477211000\end{array}$

\begin{tabular}{|c|c|c|c|}
\hline \multicolumn{4}{|c|}{ D) $)_{2} \bullet^{-}$PSG $\bullet \mathrm{CSP}^{-} \bullet \cdot \mathrm{K}\left(\mathrm{H}_{2} \mathrm{O}\right)_{5}$} \\
\hline & 5.424919 & -3.473238 & 1.45523 \\
\hline & & & \\
\hline & & & -0.689652 \\
\hline & & & \\
\hline & & & -0.3 \\
\hline & 6.719793 & -0.9 & -1.2 \\
\hline & 4.52 & -0.1 & -0.657542 \\
\hline & 3.252759 & -1.004 & -0.537196 \\
\hline & & & -0.604973 \\
\hline & & & \\
\hline & 9.023326 & -0.012545 & -1.66088 \\
\hline
\end{tabular}




$\begin{array}{lccc}7 & 2.401949 & -0.560232 & 0.549375 \\ 6 & 0.963176 & -0.712116 & 0.360561 \\ 8 & 0.446988 & -0.645564 & -0.750952 \\ 7 & 0.123408 & -0.845277 & 1.488304 \\ 6 & 0.665605 & -0.900331 & 2.722148 \\ 7 & -0.209254 & -1.122114 & 3.761980 \\ 6 & 2.095664 & -0.831860 & 2.927138 \\ 6 & 2.918648 & -0.728090 & 1.853020 \\ 8 & 7.275662 & 1.479909 & -0.378388 \\ 6 & 7.181525 & 2.197036 & -1.572621 \\ 6 & 6.414155 & 3.502434 & -1.336795 \\ 8 & 5.123606 & 3.219459 & -0.839030 \\ 6 & 7.136573 & 4.437862 & -0.363951 \\ 8 & 7.880519 & 5.415449 & -1.042089 \\ 6 & 6.002236 & 5.063387 & 0.389421 \\ 6 & 4.928663 & 3.998715 & 0.318439 \\ 1 & 5.894333 & -4.234374 & 1.885345 \\ 1 & 5.164031 & -4.580966 & -0.260817 \\ 1 & 6.711947 & -3.656336 & -0.152419 \\ 1 & 5.045485 & -2.687858 & -1.779961 \\ 1 & 5.915659 & -1.087640 & 0.683681 \\ 1 & 4.598937 & 0.267052 & -1.686320 \\ 1 & 4.548739 & 0.691313 & 0.067568 \\ 1 & 2.716890 & -0.926177 & -1.509767 \\ 1 & 0.139924 & -0.912497 & 4.665142 \\ 1 & -1.199673 & -0.966430 & 3.616586 \\ 1 & 2.506811 & -0.886329 & 3.941165 \\ 1 & 4.006419 & -0.705381 & 2.010422 \\ 1 & 8.196043 & 2.446760 & -1.956749 \\ 1 & 6.635602 & 1.612863 & -2.348538 \\ 1 & 6.292748 & 4.015360 & -2.317561 \\ 1 & 7.783910 & 3.879992 & 0.353653 \\ 1 & 8.694630 & 4.960555 & -1.381952 \\ 1 & 5.648121 & 5.985676 & -0.124021 \\ 1 & 6.281034 & 5.298667 & 1.439779 \\ 1 & 4.990802 & 3.337450 & 1.210330 \\ 1 & 3.920721 & 4.464606 & 0.291978 \\ 19 & 11.546654 & -1.037156 & 0.141090 \\ 8 & 9.938153 & -2.657966 & -2.136738 \\ 1 & 9.641486 & -2.980608 & -1.260251 \\ 1 & 9.523680 & -1.769417 & -2.207814 \\ 8 & 10.555564 & 0.885483 & 1.804495 \\ 1 & 9.635085 & 0.568499 & 1.684635 \\ 1 & 10.679824 & 1.507829 & 1.063534 \\ 8 & 12.611857 & -2.387857 & -1.849574 \\ 1 & 11.684031 & -2.598406 & -2.134324\end{array}$




$\begin{array}{lccc}1 & 13.137377 & -2.359471 & -2.660555 \\ 8 & 9.535511 & -2.853510 & 0.674280 \\ 1 & 9.192651 & -3.574417 & 1.221764 \\ 1 & 8.934019 & -2.071920 & 0.825572 \\ 8 & 11.153969 & 1.471608 & -0.944014 \\ 1 & 10.345314 & 1.064505 & -1.352164 \\ 1 & 11.455842 & 2.151758 & -1.562620 \\ 7 & -6.619554 & 0.291129 & 0.598540 \\ 6 & -6.990291 & 0.449083 & 1.959277 \\ 7 & -5.935771 & 0.211543 & 2.749459 \\ 6 & -4.845010 & -0.107097 & 1.927080 \\ 6 & -3.477870 & -0.432138 & 2.246328 \\ 8 & -2.912874 & -0.502682 & 3.340231 \\ 7 & -2.661616 & -0.710937 & 1.100118 \\ 6 & -3.171140 & -0.631661 & -0.216540 \\ 7 & -2.330063 & -1.031016 & -1.273988 \\ 7 & -4.443975 & -0.304920 & -0.515917 \\ 6 & -5.262111 & -0.054315 & 0.583726 \\ 6 & -9.642054 & 2.538049 & 1.041159 \\ 6 & -9.360246 & 1.896406 & -0.332237 \\ 8 & -7.948249 & 1.827410 & -0.560563 \\ 6 & -9.959989 & 0.452499 & -0.411479 \\ 6 & -8.715216 & -0.453417 & -0.610841 \\ 6 & -7.499966 & 0.484228 & -0.587939 \\ 8 & -9.239432 & 3.877365 & 1.135033 \\ 6 & -13.306293 & 1.710581 & 0.477745 \\ 8 & -12.539670 & -1.137680 & -0.064559 \\ 15 & -12.500169 & -0.211714 & -1.252175 \\ 8 & -10.838313 & 0.344035 & -1.470740 \\ 8 & -12.997177 & -0.590943 & -2.610150 \\ 8 & -13.236407 & 1.312465 & -0.838402 \\ 1 & -7.993875 & 0.749796 & 2.294493 \\ 1 & -1.651814 & -0.857667 & 1.229342 \\ 1 & -2.633190 & -0.723497 & -2.169644 \\ 1 & -1.340976 & -0.894622 & -1.117539 \\ 1 & -10.736873 & 2.581036 & 1.221919 \\ 1 & -9.179759 & 1.945534 & 1.863756 \\ 1 & -9.721718 & 2.555973 & -1.153237 \\ 1 & -10.474581 & 0.193578 & 0.546505 \\ 1 & -8.760124 & -0.992317 & -1.576592 \\ 1 & -8.665516 & -1.238773 & 0.165904 \\ 1 & -6.863616 & 0.405981 & -1.500019 \\ 1 & -8.323914 & 3.913924 & 0.886312 \\ 1 & -13.953986 & 2.595134 & 0.503477 \\ 1 & -12.314588 & 1.997988 & 0.872419 \\ 1 & -13.733330 & 0.939679 & 1.132554\end{array}$




$\begin{array}{lrrr}8 & -13.699696 & -3.577721 & 0.135089 \\ 1 & -13.913885 & -3.636379 & -0.792777 \\ 1 & -13.248032 & -2.728130 & 0.262712 \\ 8 & -14.279493 & -2.864761 & -3.348161 \\ 1 & -14.191635 & -3.274879 & -2.491131 \\ 1 & -13.852803 & -1.995797 & -3.285052\end{array}$

\begin{tabular}{|c|c|c|c|}
\hline \multicolumn{4}{|c|}{$\left(\mathrm{H}_{2} \mathrm{O}\right)_{2} \bullet^{\bullet-P S G} \bullet \bullet \mathrm{CSP}^{-} \bullet \bullet K\left(\mathrm{H}_{2} \mathrm{O}\right)_{6}$} \\
\hline 8 & 5.659158000 & -3.214978000 & 1.031686000 \\
\hline & 5.813689000 & -3.356908000 & -0.354255000 \\
\hline & 5.032967000 & -2.265594000 & -1.096688000 \\
\hline & 3.684294000 & -2.273640000 & -0.694258000 \\
\hline & 5.591801000 & -0.894650000 & -0.863453000 \\
\hline & 6.598432000 & -0.585119000 & 3068000 \\
\hline & 4.406642000 & -0.006657000 & 1818000 \\
\hline & 3.211118000 & -0.952940000 & 7000 \\
\hline 5 & 7.746591000 & 0.459151000 & 2000 \\
\hline & 3702000 & 965000 & 000 \\
\hline & 5339000 & 148000 & 000 \\
\hline & 352000 & -0.59 & \\
\hline & 34000 & -0.77 & \\
\hline & 6000 & 566000 & -0.9 \\
\hline 7 & 57000 & -0.9 & \\
\hline & 0.71 & -1.0 & 000 \\
\hline 7 & 669000 & -1.2 & 000 \\
\hline & 153294000 & -0.91 & 000 \\
\hline 6 & 0 & -0.76 & \\
\hline & 000 & & -0.7 \\
\hline 6 & 0954000 & 2.87 & \\
\hline & & 4.0 & -1.1 \\
\hline & 3639000 & 3.68 & -1.2 \\
\hline 6 & 69000 & 4.54 & 00 \\
\hline & 0354000 & 5.61 & \\
\hline 6 & 3327000 & 20000 & 000 \\
\hline & 3391000 & 761000 & -0.0 \\
\hline 1 & 6.186923000 & -3.947154000 & 000 \\
\hline 1 & 5.429608000 & -4.352494000 & 6000 \\
\hline 1 & & -3.292535000 & -0.6 \\
\hline 1 & 5.072433000 & -2.497016000 & 8000 \\
\hline 1 & & -0.819058000 & \\
\hline 1 & 4.389123000 & 0.477388000 & -1.994601000 \\
\hline 1 & 4.409920000 & 0.799068000 & 6000 \\
\hline 1 & 2.635329000 & -0.904260000 & -1.796610000 \\
\hline 1 & 0.256687000 & -1.054588000 & 4.456084000 \\
\hline 1 & -1.113873000 & -1.114869000 & 3.450773000 \\
\hline 1 & 2.598357000 & -0.979249000 & 3.658631000 \\
\hline
\end{tabular}




$\begin{array}{lccc}1 & 4.032415000 & -0.728525000 & 1.681241000 \\ 1 & 8.109719000 & 3.196235000 & -1.456730000 \\ 1 & 6.958767000 & 2.678397000 & -2.529756000 \\ 1 & 6.394294000 & 4.876777000 & -1.827117000 \\ 1 & 6.787097000 & 3.722789000 & 0.993030000 \\ 1 & 8.260288000 & 5.213407000 & 0.208884000 \\ 1 & 4.922763000 & 6.063787000 & 0.466420000 \\ 1 & 4.931303000 & 4.864663000 & 1.837082000 \\ 1 & 3.880175000 & 3.218882000 & 0.527458000 \\ 1 & 3.245347000 & 4.662599000 & -0.348872000 \\ 19 & 10.381863000 & -1.947240000 & -0.519383000 \\ 8 & 10.591812000 & 1.275442000 & 1.146080000 \\ 8 & 9.137730000 & -2.002955000 & -2.966950000 \\ 8 & 8.501113000 & -1.978337000 & 2.239947000 \\ 8 & 11.248046000 & 0.571017000 & -1.490714000 \\ 1 & 9.663143000 & 1.017253000 & 0.946887000 \\ 1 & 11.004643000 & 1.244829000 & 0.255004000 \\ 1 & 8.235314000 & -2.357658000 & -2.955292000 \\ 1 & 8.983332000 & -1.032682000 & -3.005656000 \\ 1 & 9.415834000 & -1.684004000 & 2.447379000 \\ 1 & 8.189387000 & -1.292788000 & 1.611782000 \\ 1 & 10.337781000 & 0.725489000 & -1.869510000 \\ 1 & 11.867318000 & 0.964537000 & -2.122840000 \\ 7 & -6.633256000 & 0.179337000 & 0.632167000 \\ 6 & -6.959998000 & 0.314676000 & 2.006625000 \\ 7 & -5.880831000 & 0.063841000 & 2.758431000 \\ 6 & -4.816787000 & -0.239782000 & 1.896196000 \\ 6 & -3.439468000 & -0.567403000 & 2.165826000 \\ 8 & -2.839447000 & -0.655142000 & 3.239518000 \\ 7 & -2.659754000 & -0.824316000 & 0.989281000 \\ 6 & -3.211211000 & -0.722212000 & -0.308623000 \\ 7 & -2.402983000 & -1.099094000 & -1.399100000 \\ 7 & -4.493874000 & -0.393401000 & -0.561458000 \\ 6 & -5.276531000 & -0.163855000 & 0.567999000 \\ 6 & -9.607572000 & 2.451606000 & 1.207896000 \\ 6 & -9.377174000 & 1.839208000 & -0.188336000 \\ 8 & -7.974506000 & 1.751676000 & -0.461257000 \\ 6 & -10.004323000 & 0.407989000 & -0.283838000 \\ 6 & -8.779238000 & -0.516431000 & -0.517120000 \\ 6 & -7.548948000 & 0.401915000 & -0.521695000 \\ 8 & -9.172638000 & 3.779111000 & 1.323916000 \\ 6 & -13.301098000 & 1.768483000 & 0.653785000 \\ 8 & -12.640206000 & -1.105081000 & 0.085481000 \\ 15 & -12.574164000 & -0.170870000 & -1.089435000 \\ 8 & -10.899356000 & 0.333241000 & -1.332162000 \\ 8 & -13.107799000 & -0.522643000 & -2.447575000\end{array}$




$\begin{array}{cccc}8 & -13.252536000 & 1.378872000 & -0.665905000 \\ 1 & -7.952168000 & 0.609843000 & 2.378583000 \\ 1 & -1.645220000 & -0.967475000 & 1.083405000 \\ 1 & -2.737311000 & -0.779651000 & -2.279325000 \\ 1 & -1.410219000 & -0.957418000 & -1.272499000 \\ 1 & -10.696006000 & 2.514093000 & 1.418917000 \\ 1 & -9.136769000 & 1.828404000 & 2.002513000 \\ 1 & -9.750584000 & 2.524832000 & -0.982119000 \\ 1 & -10.510677000 & 0.141177000 & 0.676385000 \\ 1 & -8.856879000 & -1.052144000 & -1.482527000 \\ 1 & -8.722694000 & -1.304465000 & 0.256534000 \\ 1 & -6.944482000 & 0.326784000 & -1.455454000 \\ 1 & -8.263181000 & 3.801652000 & 1.052280000 \\ 1 & -13.910092000 & 2.679811000 & 0.689670000 \\ 1 & -12.296743000 & 2.009587000 & 1.047579000 \\ 1 & -13.758864000 & 1.010687000 & 1.303067000 \\ 8 & -14.024147000 & -3.475472000 & -0.137295000 \\ 1 & -14.147696000 & -3.467970000 & -1.091428000 \\ 1 & -13.514415000 & -2.682823000 & 0.088497000 \\ 8 & -14.337247000 & -2.898579000 & -2.828553000 \\ 1 & -15.216065000 & -2.544512000 & -2.911376000 \\ 1 & -13.732032000 & -2.138535000 & -2.753147000 \\ 8 & 9.278998000 & -4.031253000 & 0.664079000 \\ 1 & 8.871382000 & -3.425104000 & 1.338529000 \\ 1 & 8.716174000 & -4.816529000 & 0.633968000 \\ 8 & 11.175906000 & -1.145781000 & 2.040554000 \\ 1 & 11.844522000 & -1.206149000 & 2.738002000 \\ 1 & 11.062533000 & -0.168306000 & 1.836648000\end{array}$

